We thank the reviewers for their helpful comments. Our responses to each comment are included below in blue. We apologise for the length of time it has taken us to resubmit our revision. To properly address reviewer concerns required additional analyses that turned out to be quite time consuming. Additionally, and more recently, an intensive campaign of fieldwork by the first author in Brazil became a more immediate and immovable priority. We appreciate the time taken by the reviewers to assess our manuscript, and we believe that they have helped us to provide a more robust and balanced manuscript.

\title{
REVIEWER COMMENTS TO AUTHOR
}

\section{Referee: 1}

Comments to the Author

This manuscript by Faria and colleagues focuses on two genera of Collembola to test for signatures of Pleistocene persistence in glaciated vs. unglaciated areas of Great Britain. I believe that this is a compelling and wellwritten manuscript, which will be of broad interest to the readers of the Journal of Biogeography. However, I would like to express a few concerns and some suggestions that may help the authors to improve it further.

\footnotetext{
- OTU delineation: OTU delineation is a very important aspect of this study as all subsequent analyses are based on the delimited OTUs. Given that it is such a critical part of the analyses, I would expect a more thorough approach, rather than relying simply on a $4 \%$ pairwise $p$-distance threshold. It is not clear why the authors did not assess different threshold values and/or they did not apply any phylogeny-based method (e.g., PTP or GMYC analyses for single-locus species delimitation). At least I would appreciate a
} 


\section{better justification and discussion on this issue, so that it does not sound like an arbitrary decision.}

The reviewer's concern is pertinent, particularly because the details about our approach for OTU delineation were not included in the manuscript. Our approach was based on prior data available from several studies that have investigated the temporal-spatial aspects and biological significance of cryptic molecular lineage diversity in the genus Lepidocyrtus, one of the two genera investigated in the present study. The more relevant of these for the present study is Cicconardi et al. (2013) who defined a molecular lineage within a morphological defined species of Collembola as "a group of individuals, or an individual, that are reciprocally defined as a monophyletic (group of individuals) or as a divergent lineage (single individuals) by unlinked loci." They used Hardy-Weinberg equilibrium (HWE) and linkage-disequilibrium (LD) tests among lineages in sympatry to evaluate molecular lineages within morphospecies. As a result, they found 19 molecular lineages occurring sympatrically, with genetic isolation in sympatry congruent with assignment to biological species. We then based our OTU threshold on these prior data and the thorough approach behind with explicit reference to the biological species concept. We used sequences from these 19 molecular lineages of Cicconnardi et al. collected in sympatry to calculate their pairwise distances and used the minimum p-distance found between them (0.04) to define our $96 \%$ similarity threshold. This is now summarized in lines $180-190$.

Initially, we did not apply any phylogeny-based method due to the nature of Collembola data, which mostly present deep divergent sequences that may have experienced high levels of saturation. This is not very appropriate for Bayesian or ML methods. Thus, we used a NJ method to build the tree and used, as explained above, prior data regarding species definition available for Collembola to define the $4 \%$ distance threshold. That is to say, our interest was to identify clusters, not higher-level relationships among clusters. 
We are aware that $\mathrm{NJ}$ uses a genetic distance-clustering algorithm without an optimality criterion. Thus, we have taken on board the reviewer's suggestion to have a more thorough approach to define OTUs, and explored Bayesian Inference $(\mathrm{BI})$ and Maximum Likelihood $(\mathrm{ML})$ to build trees to then explore a range of OTU delimitation methods. Due to limitations and caveats inherent to delimitation methods, we applied three different approaches to then assess congruence of inference across approaches (explained in lines 155-190).

Main limitations:

GMYC - performance is affected by: ratio of population sizes to species divergence times, varying population sizes, number of species involved and number of sampling singletons (tends to over split species) bPTP and MPTP - branch lengths proportional to the amount of genetic change rather than to time. Outperforms GMYC when interspecific distances are small.

First, to infer an ultrametric tree, which is a requirement for the GMYC method, we ran BEAST analyses using all sequences but no outgroups, since they tend to compress the coalescent events towards the tips of the trees, making difficult for GMYC to distinguish closely related species. We tested a combination of different parameters (tree and clock models) and priors (for the ucdl.mean) but BEAST analyses were somewhat problematic (there were convergence issues with poor ESS values). These problems are likely to be due to the type of data (species level data with some variation within species), which cannot be fully resolved by a Yule tree prior or by a Coalescent tree prior. As we were not confident on the resulting BI trees (which actually resulted in very different GMYC results), we explored ML analyses.

We inferred a gene tree using maximum likelihood in RAXML (Stamatakis et al., 2008). After rooting the tree and removing the outgroup sequence, we used the inferred tree for species delimitation by bPTP and MPTP methods (Zhang et al., 2013; Kapli et al., 2017). However, bPTP failed to run the raxml 
tree, possibly because the branch lengths of ML trees seemed to be set to the minimum. This could be potentially a problem caused by the large amount of exactly identical sequences across both datasets. To take this into account, we collapsed our sequences into unique haplotypes, and re-run ML analysis and bPTP analysis with this shorter dataset. However, bPTP also failed to run and we focused on MPTP, an improved model over bPTP that accounts for different levels of intraspecific genetic diversity. It also implements a more accurate heuristic algorithm compared to bPTP. Both Entomobrya and Lepidocyrtus unique haplotype datasets were successfully run in the webserver. Using the multicoalescent rate, mPTP delimited 12 Entomobrya and 17 Lepidocyrtus OTUs for ML trees.

Finally, we re-ran Beast analyses using the unique haplotype dataset for both genera. Optimal convergence and ESS values were achieved and resulting BI ultrametric trees were used for both GMYC and MPTP analyses. GMYC was run in R using the 'splits' package (Ezard et al., 2009). Number of GMYC ML entities were 22 for Entomobrya and 18 for Lepidocyrtus. Finally, mPTP for the $\mathrm{BI}$ tree defined 13 OTUs for Entomobrya, and 22 OTUs for Lepidocyrtus. Details of similarities and differences among OTUs defined by the different methods are in Appendix 2: Table S2.2.

In general, our previous NJ tree inference was similar to the $\mathrm{BI}$ haplotype trees and only slightly different from ML haplotype trees (see Appendix 2:Table S2.1). OTU delimitation methods slightly differ in number of OTUs defined, thus we have taken a conservative approach counting OTUs as those that were recovered in the majority of approaches employed and avoiding intra splits (Appendix 2: Table S2.2). Final number of OTUs is 12 for Entomobrya and 18 for Lepidocyrtus.

\section{- $\quad$ NJ trees: On a related note, I do not find very appealing the fact that the only trees presented in the manuscript are distance-based neighbour- joining trees, which are however treated as if they were true phylogenetic}


trees (e.g., they are used to establish monophyletic sequence clusters - lines 271,361 etc). To establish monophyly, I think it would be preferable to include a phylogenetic tree (e.g., a Maximum Likelihood analysis using RAxML which is particularly suitable for large datasets). Or you could just present the BEAST trees (if they included the full dataset, see next point below), which are currently not presented in the manuscript or supplementary material.

\begin{abstract}
As explained above, we have substituted the $\mathrm{NJ}$ trees with BEAST unique haplotype trees (Figs. $2 \& 3$ ) and included all haplotypes ML trees in the supplementary material (Appendix 2: Figs. S2.1 \& S2.2).
\end{abstract}

BEAST trees used for dating analysis did not include the full dataset, only the geographic localized monophyletic OTUs and they are now included in the supplementary material (Appendix 4: Figs. S4.1 to S4.7)

- Dating analyses: The methodology followed for the dating analyses in BEAST (lines 180-185) is not very clearly explained. For example, it is not clear which datasets were used for these analyses. If I judge from the explanations in the Results section (lines 333-343) it appears as if the analyses were applied separately on specific sequence clusters (as the models of molecular evolution are reported for each OTU or sequence cluster separately). However, if this is the case, I do not understand why would you opt for a Yule speciation prior for the analyses (lines 183-184), given that you are basically analysing intraspecific datasets. Please clarify and justify which datasets were used for these analyses. It would be also particularly helpful to include the BEAST trees in the figures of the manuscript or the supplementary material.

Yes, dating analyses were applied separately for the geographically localized monophyletic clusters and this has been clarified in the text (lines 221-222). 
We agree with the reviewer's point that the Yule speciation prior was inappropriate because we were in fact analysing single OTUs with coalescent variation. Thus, dating analyses have been repeated with the Coalescent tree prior (lines 224-225). Estimated ages are older than the ones previously found using the Yule tree prior, thus, our conclusions have not been changed (lines 406-408, 413-414 and Table 5).

- $\quad$ Evolutionary rate estimation: I think you should also clarify and justify better the strategy used for estimating the applied evolutionary rate. For example, it is not very clear: A) Why do you need to use a COll external rate to calibrate the COI rate? (instead of using directly a COI external rate). It would be good to explain that from the beginning of this section, otherwise it is quite confusing. B) How does your estimate for the COI rate compares with the rates cited in the literature you mention? It would be good to explain that too. C) In lines 165-166, why do you mention the broad range of values found in the literature for the COIl gene, but then you do not really use this range for anything, not even for the discussion. Also, the literature you cite in lines 166167 does not seem to focus on the COll gene, but they are more general about mitochondrial clock rates. D) Is it justified to calibrate your evolutionary rate based on old divergence times (35Ma here, line 176) and then use it for estimating very recent events?

We have included a more thorough explanation about the strategy used. See lines 196-228.

Answering each point raised:

A) Currently, there is no COI rate available for Collembola. However, a COII mean rate has been estimated for Lepidocyrtus in an extensive comparative rate analysis using COII sequences found across Pancrustacean major lineages (Cicconardi et al., 2010). We make use of this rate for calibrating the 
rate of the COI region, as it is a rate specifically estimated for one of the genera that we are working on.

B) We have only mentioned that we are using a fast and more conservative rate of evolution. We have not compared our COI rate because rates cited in the text were proposed to insect groups (e.g. beetles, butterflies, drosophila).

C) We mentioned the broad range of rates already proposed for the arthropod mtDNA to give a general idea of how this rate can vary across groups and we did not discuss how our $\mathrm{COI}$ rate fits in this range because it comes from a different class (Collembola). References used in these lines are indeed more general about the mtDNA molecular clock and we have changed them to more appropriated ones.

D) Indeed, rates of mitochondrial evolution have been suggested to be time dependent in a variety of taxa, with a trend of declining rates over time for insect mitochondrial DNA, but there is in fact little supporting evidence, with time calibrated DNA analyses recently revealed to suffer from inherent bias (see https://doi.org/10.1111/mec.13451). By using a rate obtained from an old estimate (35 Ma), we found a rate that is faster than those universal rates (0.006-0.0248\%) discussed above, including the COII rate estimated for Collembola. Thus, we are more likely to be underestimating than overestimating the ages of divergence we are interested in. Then, if using this faster than expected rate, we obtained ages that predate glaciation periods, we can be confident that this conservative approach provides reliable relative time frames for lineage persistence over long periods of time.

- $\quad$ Structuring of genetic variation: An important aspect for the interpretation of the observed patterns seems to be the "non-random geographical structuring of genetic variation" (e.g., lines 270, 294-296, 359$360,363)$. However, it is not clear how the authors evaluate the existence of a non-random structuring. There is a description of the observed patterns and maps are used for presentation which is nice, but I do not see any formal 
analysis or statistical test. It would be helpful to include some kind of test to assess the structuring of genetic variation, it could just be some simple analysis (e.g., AMOVA- Analysis of Molecular Variance)

\begin{abstract}
Yes, this is a good point, as we have not quantitatively evaluated the existence of non-random distribution of monophyletic clusters. This has been only visually inspected because testing for significance is not simple. AMOVA would not be the way to go because it only tells about the existence of population differentiation and the pattern we are really interested in it is local monophyly.
\end{abstract}

To address the reviewer's concern, we have applied Ripley's function (K) to our data (Dixon, 2002). This method determines spatial distribution of point patterns, i.e. OTUs, to check whether they appear to be dispersed, clustered or randomly distributed. For inferential purposes, the estimate of $\mathrm{K}$ is compared to the true value of $\mathrm{K}$ for a completely random (Poisson) point process. Deviations between the empirical and theoretical $\mathrm{K}$ curves may suggest spatial clustering or spatial regularity.

K function was tested using the package 'spatstat' in R (Baddeley \& Turner, 2005). Three corrections ( $\mathrm{K}_{\text {iso }}$ - isotropic, $\mathrm{K}_{\text {trans }}$ - translation correction, $\mathrm{K}_{\text {bord }}-$ border method) were applied to observed data while estimating $\mathrm{K}$ function and these were compared to what it would be expected if spatial distributions were completely random ( $\mathrm{K}_{\text {pois }}$ - completely random Poisson point process). For both Entomobrya and Lepidocyrtus, empirical K functions for overall OTUs deviate from the theoretical expected value.

These plots used overall distribution of all OTUs, we then checked patterns for individual OTUs of interest (localized and disjunctly distributed clusters) using the markfunction and then running Kdot (i-to-any) function. This is the analogue to $\mathrm{K}$ function but for individual marked OTUs (multitype data). To run Kdot, an i mark value (character string identifying the points in X from which distances are measured) was defined as each OTU of interest and 
analyses run separately [i.e. analyses ran between localities of occurrence of a defined monophyletic cluster (I) versus localities where all other OTUs were sampled (to-any)].

Plots for geographically localised monophyletic OTUs within Entomobrya and Lepidocyrtus show significant deviation from random distribution consistent with geographic clustering. Kdot function analysis was added to the text (lines 236-243 and 370-373) and we present plots for OTUs of interest (localized and disjunctly distributed) in the supplementary material (Appendix S3: Figs. S3.5 \& S3.6).

- $\quad$ Limited dispersal capability: Both in the introduction and in the discussion the authors support a very low dispersal capability for these genera of Collembola (lines 98-99 and 376-380), which is an important argument to discard a scenario of long-distance dispersal after ice-sheet retreat (lines 373-376). How is this compatible with the fact that there are almost identical haplotypes distributed in Canada, South Africa or Australia (lines 242-243 and 259)? Please explain this in the discussion.

This can be reconciled as follows (added to the text in lines 470-482):

Great Britain sequences that are genetically identical or nearly identical to sequences sampled in remote continents are most plausibly explained as human introductions. Passive dispersal of Collembola through human activity has been implicated in the sharing of lineages across distant continents, e.g. Cerathophysella denticulata lineage L3 found in Canada, South Africa, Australia and New Zealand (Porco et al., 2012), and such introductions are considered to have mainly been accidental (e.g. in soil used as ship ballast). Mitochondrial metagenomic data has also revealed island faunas to be highly represented by introduced Collembola (Cicconardi et al. 2017). Our data set presents similar examples, such as Entomobrya OTU 6, with sequences $100 \%$ similar sampled in Canada, and South Africa and sequences 100\% identical to Lepidocyrtus OTU 9 sampled in Australia and Tasmania. Genetic 
identity, or near identity across these vast geographic distances is consistent with passive transport through human activities

- $\quad$ Sampling effort: I think it would be helpful to provide some more details about the sampling (lines 119-121). The reader may wonder how did you ensure equivalent sampling in glaciated vs. non-glaciated areas and at each sampling site.

We added the following paragraph to Methods, lines 119-126

Samples were collected from 98 sites across Britain between 2011 and 2012 . The aim was an approximately equal sampling effort across the glaciated and unglaciated areas of England and Wales, collecting particularly from the geographical extremes of the mainland (Fig. 1, Appendix 1: Table S1.1). At each location, surface-active animals were collected by vacuum collection (two sets of 30 second collections, transferred directly to EtOH), and duplicate soil / litter samples were collected with minimal disturbance into plastic bags, for return to the laboratory and extraction in a Tullgren funnel system followed by manual removal of the relevant genera.

Some typos and small corrections/clarifications (not an exhaustive list):

- Line 180: "This estimated COI rate of was" remove "of"

Changed.

- $\quad$ Line 136 vs. Line 232: The difference in the fragment lengths is due to trimming the ends of the sequences? Please clarify

Yes, primers ColFol-for and ColFol-rev amplify a 658bp barcode region, however, when sequencing our DNA samples, the final ends of the sequences did not present clear peaks, so they have been trimmed. 


\section{- $\quad$ Line 402: change "these result suggests" to "these results suggest" Changed. \\ - Line 446: change "bases" to "based" \\ Changed.}

Referee: 2

Comments to the Author

The present study evaluates the predictions of the "southern richness, northern poverty" hypothesis in 2 genera of a group of terrestrial invertebrates with restricted dispersal abilities. The assessment of genetic diversity is done with the amplification of $\mathrm{COI}$ and morphospecies assignment based in matching the genetic data with a reference panel of identified specimens. Regardless the morphospecies assignation specimens are clustered in reciprocally monophyletic groups (OTUs) based on genetic distance with reference to a previous study of sympatric species from Panamá. The main result is the evidence of persistence of Collembola during Pleistocene glacial cycles in the northern areas of the British Isles. By showing pre-LGM OTUs clades, the authors provide attractive evidence of potential geothermal glacial refugia on areas previously covered by the ice. This idea is supported in recent evidence from Antartica.

The strengths of the study are: (1) ask a general question on an understudied group; (2) an extensive sampling in terms of geography and number of specimens; and (3) provide an alternative hypothesis (geothermal glacial refugia) to explain the presence of old lineages in glaciated areas. Points to be improved on the manuscript: (1) refer explicitly to situations that deviate from the general trend (i.e. (i) OTU 1 of Lepidocyrtus been more diverse in glaciated areas, or (ii) no explanation to the potential barriers that create disjunct distributions on Lepidocyrtus (see Fig 5a-b)); (2) presentation of phylogenies (see note on Fig. 3); (3) discussion of alternative mechanisms 
to explain genetic patterns observed (for example: (i) differential expansion speeds for both genera (Entomobrya faster than Lepidocurtys), or higher genetic diversity in the south for Lepidocyrtus due to historic man mediated introductions from other parts of the world); (4) justify some technical decisions on the data analysis (program to select models of molecular evolution, or application of OTUs specific models of molecular evolution in the estimation of MRCA); and (5) lack of discussion in terms of the implications for the tested hypothesis of the presence of 2 intra-OTU clades (NIVG1 and NVG2) on Entomobrya. Please, also refer to the Specific comments for more detailed suggestions.

I would recommend this article as: "Accepted with minor revisions".

Specific comments:

Line 175: Why did you choose those models? Why they are different on each gene?

Choosing the most appropriate model of DNA sequence evolution for a DNA sequence dataset is a crucial step for inferring trees and estimating times of divergence. We determined the most appropriate model for each dataset using maximum likelihood approach in MEGA software.

Substitution models are different on each gene because sequences obtained differ in many aspects (e.g. base frequencies, GC content, bias in transitions and tranversions, etc.), which affect the rate of change from one nucleotide to another. Models take these differences in account by incorporating different rate parameters, thus, different genes are likely to result in different substitution models.

Line 223: Are they "morphospecies" or properly identified species? The fact that they are assigned to scientific names makes it look like they were properly assigned to described species. Please, make this clear. 
The original aim of the work was to establish the extent to which morphospecies and genetic species agreed in these two genera of Collembola. It was important to minimise damage to the animals' bodies prior to DNA extraction, so standard additional checks such as squashing mouthparts had to be avoided. Inevitably this means that any names generated prior to sequencing must be morphospecies. Whether the referee regards these as "properly identified species" or not is almost a semantic question.

To expand on the process: the animals were allocated a species name based on the result of keying them out using primarily one source, Hopkin (2007). For Entomobrya this key relies almost exclusively on colour pattern, and is not fully adequate as in conflates E. nivalis and E. intermedia (Steve Hopkin was not convinced that $E$. intermedia was a good species while writing this key), so once an Entomobrya was keyed to E. nivalis its dorsal pattern (especially dark markings on abd 4-6) was further checked against South (1961). Lepidocyrtus were keyed out using the same key, but in this genus the key uses morphology as well as colour patterns. The shape of T2 and the distribution of scales on legs and antennae are especially important, although the scales are an unsatisfactory feature since they are hard to see in uncleared specimens, dislodge easily on handling, and can wash off the body to end up apparently associated with the legs.

\section{Line228: Same comment as with Entomobrya.}

Please, see answer above.

Line 243: How do you discard the presence of introduced haplotypes? That is suggested by the geographic origin of the sequenced haplotypes. 
Rather than discarding, we have acknowledged the presence of introduced haplotypes and provided a better discussion regarding human introductions, as explained above in answer to referee 1 (see lines 470-482)

Line 334: The Methods section does not indicate the program used to select this. Please, add.

Methods did indicate it, now in lines 222-223 (MEGA)

Line 340: The fact that 2 different models of molecular evolution were selected for 2 different OTUs implies that a different time-calibrated phylogeny was used to estimate the age of the OTU on each case, is it correct? If yes, what models were used for the other OTUs?

We have only estimated the age of OTUs that are geographically localized to a restrict area and this was done for each OTU separately. As it has been explained in lines 413-414, the K2 model was selected for both OTUs 3 and 8 , while T92 model was selected for three OTUs: 7, 12 and 18 (all models selected using MEGA with each dataset separately).

If my understanding is correct, I would say that the use of different models of molecular evolution to estimate time-calibrated phylogenies within the same group is an unusual practice. I am afraid that the use of different models might affect the possibility to compare the age estimations. I would use the same model for the whole phylogeny and then compare the estimated ages. However, I do see the benefit of using clade-specific models, as there could be variability between clades on the rate of evolution.

I think this section would benefit by an explicit explanation of the rational of this decision and if there is another paper where this approach is used, please include it as a reference.

We have not estimated ages for the whole phylogenetic tree. Our aim was not to provide a time-calibrated phylogeny for the group but rather provide a 
relative temporal framework for coalescent origins of geographically localized monophyletic clusters as they indicate endemic genetic variation evolving in situ and check whether they predate the LGM. We are not aware of another paper using a similar approach but we have expanded our explanation in lines 196-228.

Line 359: How do you interpret that only one OTU (OTU 4) has a non-random pattern? Why is it different?

This is only mentioned in the discussion and there is no further evaluation of its relevance for the main argument.

First, we mapped the geographic distribution of each sequence, and then we evaluated how OTUs or variation within OTUs (e.g. monophyletic clusters) are distributed across sampling sites (widespread, restricted to a few closely located sites or disjunctly distributed). When we closely examine the amount of sequence variation within each OTU and the geographic distribution of this variation, we can see that only OTU 4 has sequence variation restricted to a few proximate sites, i.e., they are geographically localized to a restricted area. While the other Entomobrya OTUs present distinct patterns, with sequences being more widely or disjunctly distributed.

Line 404: The presence of more than one morphospecies on the same mitochondrial lineage in Lepidocyrtus could be caused by introgression events. Have you considered this alternative? How this would affect your conclusions?

One of the controversies of DNA barcoding is that it can generate false negatives, i.e., identical barcodes in two different species, and this could be explained by mtDNA introgression, hybridization, and incomplete lineage sorting causing incongruences between gene trees and species trees (Funk \& Omland, 2003). To be able to evaluate introgression, we would need a 
combination of mitochondrial and nuclear genes to assess these incongruences but we have only sequenced the COI gene. However, no mtDNA introgression and rarely hybridization have been documented in Collembola (Burkhardt \& Filser, 2005). For example, field studies have shown a low probability of natural hybridasation between individuals derived from sympatric population which has been related to specific habitat preferences of these population resulting in spatial isolation, thus preventing hybridization (Skarżyński, 2004)

We believe a taxonomic issue is more likely to explain this pattern. Collembola studies are proving morphological species to be largely polyphyletic and constituted by several monophyletic clusters geographically distributed in restricted areas (e.g. Garrick et al., 2007, 2008, Cicconardi et al., 2010), and many studies are challenging the current taxonomic system which lacks enough morphological characters to reliable identify species and species boundaries (e.g. Cicconardi et al., 2013). Morphological stasis due to a possible buffering effect for the soil environment (selection does not act in visual cues) has also been used to explain some discrepancies between morphological and molecular data, such as a high level of cryptic species.

Furthermore, molecular studies indicate stable lineages evolving independently with no sharing between close located sites and long divergence time as main features for Collembola diversity (e.g. Cicconardi et al., 2010, 2017). Finally, if introgression more than taxonomic issues were to explain our data, it would not change our conclusions regarding number of OTUs and time of origins for endemic variation. It would rather tell us that some of our OTUs are a process of mixing between two putative species.

\section{Line 413: What if the most diverse areas, are more diverse because they have more introduced haplotypes? The south of the country has very important port cities.}

This is not likely to be the case because introduced haplotypes occur in both 


\section{North and South of the country.}

Line 415: Entomobrya presents the similar diversity in glaciated and unglaciated areas, while Lepidocyrtus is more diverse in unglaciated areas. What if this pattern is explained by differential re-colonization speeds between genera?

Been Entomobrya faster in recolonization and Lepidocyrtus slower. Then, given enough time, the diversity of Lepidocyrtus will be also the same between glaciated and unglaciated areas. In other words, if differential recolonization speed is taken into account, this data (both genera distributions) could still support the "northern poverty" hypothesis. Please, refer to this possibility.

This possibility has been acknowledged in the text (lines 506-508).

Table 1:

- $\quad$ Why range is not included? It is present on Table 2

\section{It has been included to Table 1 .}

- $\quad$ On the morphospecies with "-" on the last column mean that there is "no match". If yes, write that in order to have the same nomenclature as in Table 2.

This has been changed.

Table 2, Column OTU 1: It seems quite interesting that OTU 1 is more diverse on the glaciated area. It is true that corresponds to only one of 18 lineages, but it is one of the most widespread. I would suggest to, at least, referring to this situation explicitly in the discussion. Does it have an effect on the final 
interpretation of the data or it is just an isolated situation?

Table 2 refers to numbers of localities where OTU1 has been sampled in each area (glaciated $x$ unglaciated). There is no indication of haplotype diversity between these areas. This is actually referred to in Table 4. Number of haplotypes are greater in glaciated than unglaciated areas for both OTU 1 and 15 , however after rarefying the data and testing diversity differences using EcosIM, as explained in methods, we found intra-OTU richness to be equivalent in both areas for both OTUs.

Figure 3:

- $\quad$ Lineages $6 ; 9.1 ; 15.1 ; 15.3$ and 16 , could also be cartooned to save space.

This figure has been changed.

- $\quad$ Two different clades are lab

This comment is incomplete.

Referee: 3

Comments to the Author

The paper by Faria et al. has a number of positive attributes. For example, it broadly examines genetic diversity (i.e., at the genus, species and populationlevel) in organisms for which relatively little information about spatial structuring is available, and presents an interesting (and perhaps somewhat counter-intuitive) finding of pre-LGM persistence in glaciated areas, suggesting an important role cryptic northern for micro-refugia. Given this, I think that paper has potential to make a strong contribution to understanding of biogeographic patterns and processes in northern latitudes.

My main reservations relate to (1) the rather qualitative / descriptive nature of 
the inferences, (2) the use of potentially confusing / inconsistent terminology relating to units of biological organization, as well as (3) missed opportunities for extracting more information out of the data that were collected. These are expanded upon below.

1. Examination of intra-OTU spatial structure seems to have been done byeye, yet conclusions based on this a re phrased quite strongly. For Entomobrya spp. OTU 4, I think that there is sufficient sympatry between the two subgroups that the questions about the extent of substructure could be raised on the basis of differing personal opinion; for Lepidocyrtus spp. OTUs, readers cannot evaluate subgroup spatial structure for themselves, and so is an issue with transparency. In the latter case, the delineation of intra-OTU subgroups also not presented, and I don't recall seeing any explicit criteria laid out for identifying such subgroups a priori. For example, for Entomobrya spp. OTU 4, only a subset of the haplotypes in this this OTU are assigned to a subgroup, and basis of deciding which haplotypes are included or not needs clearer rationale. I would add that, if elements of the estimated phylogenetic tree topology and node support values are used for this (cf. simply using \% sequence divergence thresholds), then maximum likelihood and/or Bayesian inference would be preferable to the current $\mathrm{NJ}$ trees.

We have re-analysed our data through both Bayesian and Maximum Likelihood inferences and these trees were used as input to OTU delimitation analysis (GMYC and mPTP). Please, see the detailed explanation given in response to a similar issue raised by referee 1. Within Entomobrya OTU4, a widespread OTU, the subset of haplotypes indicated as in situ endemic variation was defined by node support $(\mathrm{BI}$ posterior $=1)$ and inspection of its distribution, e.g. monophyletic clusters occur only in closely located geographic sites as opposed to the wider distribution of other sequences belonging to this OTU. For Lepidocyrtus, none of the widespread OTUs presented this signature, but five OTUs (defined as explained above) were restrictedly distributed across sampling sites, indicating their localized 
occurrence. Thus, we are not referring to subgroups but rather to entire OTUs within Lepidocyrtus data and reader can verify support for their monophyly and their localized distribution by looking at Figure 3 and maps presented in Figure 5.

2. Throughout, there is awkward interchangeable use of the following: (a) intra-OTU = within species = among population; (b) OTU = species = lineage . I recognize that OTUs are defined as groups of haplotype with $<4 \%$ sequence divergence among them, that these are tentatively treated as species. However, some confusion arises when also using the term species to refer to morphologically identified named species, and in some cases (owing to a departure from the standard number system used for most OTUs) "lineage" is no longer synonymous with OTU. All of this is further complicated by use of the term "communities", which in some cases is clearly misapplied (i.e., when dealing with the intra-OTU level, it would effectively be referring to communities of haplotypes), and in other cases is questionable (e.g., when dealing with multiple OTUs, it implicitly suggests that the communities are comprised only of Collembola from up to two genera).

We have revised the use of these terms throughout the text. We noticed that the confusion mainly appeared in sections related to species diversity analysis in which we erroneously used the term species to designate OTUs and referred to communities of these species in glaciated and unglaciated areas without explaining the term. The word community is used to refer to the collection of sequences of each genus found in each area. It is a statistical term used in the analysis more than an ecological term. We have added an explanation of what we mean by community in lines 262-263 and Tables 3 \& 4 captions. Apart from that, we have used OTU or intra-OTU to talk about taxonomic units defined by molecular data and avoided the use of lineage or species terminologies. Also, morphologically identified named species have been always referred to as morphospecies. 
3. Both measures of richness (i.e., rarefied number of different OTUs at the species-level, and rarefied number of haplotypes at the intraspecific-level) treat all countable entities as being equally different from one another. However, the information embedded in the DNA sequence data that could be used to consider phylogenetic diversity at both levels. Also, I think that more could be done with the finding that Entomobrya comprised of 13 OTUs, but assigned to only 6 morphospecies, and Lepidocyrtus was comprised of 22 OTUs, but assigned to only 8 morphospecies (two unnamed). Does this suggest that the $4 \%$ sequence divergence cut-off for $\mathrm{COI}$ does capture biologically relevant partitions (i.e., it over-splits groups), or alternatively, that that morphological characters are insufficient (i.e., lead to under-splitting)? If one of these explanations is favored, on what basis is that other discounted? If the favored explanation necessarily the same for both genera, and why / why not?

Measuring phylogenetic diversity (PD) gives, among other important things, information about community structuring patterns (e.g. whether species in a community present phylogenetic clustering or overdispersion) which goes beyond our main focus. Taking referee's suggestion, we have calculated phylogenetic diversity (Faith's PD) for communities of Entomobrya and Lepidocyrtus from glaciated and unglaciated areas. Then compared, through standardized effect size (SES), observed Faith's PD patterns to those expected under various null models of community assembly and phylogeny structure (Swenson, 2014). SES was run with iterations $=1000$ and runs= 999 using 'picante' package in $\mathrm{R}$ (Kembel et al., 2010). For datasets using overall OTUs, Entomobrya PD in both glaciated and unglaciated communities were significantly lower than what it would be expected in null communities (Table 1). For Lepidocyrtus, $P D$ in glaciated community does not differ from the expected in null communities, while PD in unglaciated community is significantly lower than expected in null communities (Table 1). These patterns of lower PD indicate that closely related species occur more together than expected by chance, i.e. phylogenetic clustering, which suggests niche 
conservatism. Although we have understood how PD varies in our dataset, we rather not include this to our main text because it opens a new topic and discussion that is not essential for the patterns we are describing, plus it would compromise word limit. Additionally, we are uncomfortable extrapolating phylogenetic relationships from our single marker, and as mentioned above saturation issues compromise deeper phylogenetic inference.

Regarding correspondence between morphology and molecular analysis, as discussed above, we have re-done our delimitation analysis and results are similar to the $4 \%$ cut-off used previously. The discrepancy found between morphospecies and OTUs is a common feature in studies applying molecular methods to survey biological diversity. For details and potential explanations, please refer to our response to similar question raised by referee 2 . An insufficient morphological taxonomic system is likely to explain mismatches for both genera and this had been briefly discussed in lines 494-497.

Table 1 - Faith's Phylogenetic diversity (PD) measures for Entomobrya and Lepidocyrtus datasets from glaciated and unglaciated communities. Observed patterns were compared to those expected under null models of community assembly and phylogeny structure. ComGL = community in glaciated areas, comUnG = community in unglaciated areas, n.tx = number of taxa in community, pd.obs = observed Faith's PD in community, null = mean PD in null communities, sd = standard deviation of $\mathrm{PD}$ in null communities, rank = rank of observed PD vs. null communities, obs. $p=$ P-value of observed $P D$ vs. null communities

\begin{tabular}{lccccccc}
\hline & Genus & n.tx & pd.obs & null & sd & rank & obs.p \\
& & 9 & 1,46 & 1,97 & 0,24 & 12,00 & 0,012 \\
ComGL & Entomobrya & & & & & & \\
& & 12 & 1,92 & 2,31 & 0,21 & 53,50 & 0,054 \\
ComUnG & 11 & 10,52 & 13,35 & 1,96 & 82,00 & 0,082
\end{tabular}




\begin{abstract}
L34. insert word: "... [genetic] signatures..."

L40. what would the signatures of persistence (cf. recent recolonization) look like, given the focus on OTUs within genera?

Endemism and disjunct distribution have been used as basic arguments to support the glacial survival hypothesis (e.g. Brochmann et al, 2003), thus they are considered signatures of persistence. Populations surviving glacial cycles are expected to accumulate genetic variation, and they should contain related and locally endemic alleles, distinct from surrounding regions (Hewitt, 1999). As explained throughout the manuscript, signatures of persistence were found in monophyletic clusters of sequences occurring in geographically localized sampling sites, i.e., endemic variation that diversified in situ. For Entomobrya, this was found within a widespread OTU, while for Lepidocyrtus, entire OTUs presented this pattern. Also, clear patterns of OTUs disjunctly distributed were found within both genera. Remaining OTUs that are widespread but present no sign of localized endemic variation or are geographically localized but present no sequence variation are more likely to be a result of recent recolonization.
\end{abstract}


L44-45. in what way is "geographically disjunct genetic variation" different from "geographically localized diversification of lineages"?

These patterns are quite distinct in the way genetic variation is spatially distributed. In the first pattern, related sequences are distributed considerably apart from each other in the geographical space (e.g. an OTU found in East Anglia and South West). While in the second pattern, the range of distribution of genetic variation is rather geographically restricted to a few closely located sites.

L46, what is the basis for suggesting that pre-LGM diversification occurred in situ?

As explained above, populations surviving glacial cycles are expected to accumulate genetic variation, and they should contain related and locally endemic alleles distinct from surrounding regions (Hewitt, 1999). Our finding of monophyletic clusters of sequences that are geographically restricted to a few closely located sites whose coalescent origin predates LGM, indicate endemic variation evolving in situ. Following a comprehensive approach to estimate dates for the onset of these in situ diversification events, we found ages for these events that were older than the LGM, indicating population persistence that precedes LGM.

\section{INTRODUCTION}

L57. replace word: "significantly" often implies a statistical test has been performed.

Changed to "considerably".

L60. expand abbreviation (Ma.) on first mention. Also, lower bound (0.01 Ma) 
marks the beginning of the Holocene, which is generally thought to be been free of glaciation events... should this lower bound instead be $0.02 \mathrm{Ma}$ (i.e., at the height of the LGM)?

The lower bound for LGM is 11,7Ka (e.g. Tzdesaki et al. 2013), thus, we have changed it to $0.012 \mathrm{Ma}$.

L63. Delete word: [geographical] - seems redundant, given reference to latitudinal range.

Changed to geographic position

L64. Consider replacing "leaving a" with "resulting in a present-day" Replaced.

L68. The opening of this new paragraph refers back to "this view" given in the previous one. I think the new paragraph should stand alone, by re-stating / clarifying the major elements of the scenario given previously (e.g., "local extirpation followed by recolonization from southern refugia, coupled with loss of intraspecific diversity owing to founder effects" or similar).

Due to manuscript word limit, we rather do not re-state the scenario explained in the previous paragraph. We believe it is sufficient.

\footnotetext{
L76. "Environmental stability" seems vague. Does this really just mean climatic stability / thermal buffering... or is there more to it? Either way, I think this should be rephrased.
}

Changes in water properties such as temperature and chemistry are much slower in subterranean than in surface waters. Therefore, groundwaters are 
buffered from extreme changes in temperature and rapid hydrological variations, resulting in a more stable environment.

We have added a short explanation to line 78 .

L79 \& 82. Replace word: [presents / presented]

'Presents' changed to 'shows'

L82-84. Here and above the authors seem to be suggesting that if haplotypes are presently endemic to an area, then they must have arisen there / evolved in situ. While I agree that this may be likely and is certainly a parsimonious explanation, it does not preclude a scenario in which a distinct lineage evolved elsewhere, colonized a new area, and subsequently went extinct in the original location. I think that underlying assumptions should probably be rationalized for the reader.

Yes, we agree and have suggested this when discussing patterns for disjunctly distributed OTUs. These native OTUs probably presented a broader distribution in Great Britain but went extinct in most of their ranges in response to climate change (lines 458-464). We have now explicitly raised this scenario for localised clades explaining they could also have once been widespread (i.e. same and related alleles found elsewhere), but went extinct in most of their range in response to climate change, surviving in a few icefree localities (lines 464-468)

L87. expand abbreviation (ka) on first mention.

Changed. 
L91-92. rephrase, to past tense.

We do not think it is necessary.

L104, insert words: "...test for [the following] signatures..."

Changed.

L105, What aspect(s) of "intra-OTU genetic richness" are considered (e.g., based on entire haplotypes vs. just on segregating sites, or some other)?

We have considered haplotypes within OTUs and this is explained in Methods section.

L108, replace "origins" with "times" (or similar).

We do not think it is necessary.

L109, Is long-distance dispersal considered an implausible explanation a priori (e.g., given the statements about dispersal limitation of Collembola, given earlier in the Intro), or is this still considered to be biologically possible and therefore is somehow going to be tested against alternative explanations for disjunct distributions?

Yes, it is considered unlikely to be due to dispersal limitation, as explained in Intro and Discussion, lines 450-464.

L110. expand abbreviations (MtDNA, COI) on first mention.

Changed.

\section{METHODS}


L118, Here it says $\mathrm{N}=722$ samples of Entomobrya spp. and $\mathrm{N}=428$ of Lepidocyrtus spp., but Table $\mathrm{S} 1$ lists $\mathrm{N}=724$ and $\mathrm{N}=391$, respectively.

This has been verified and corrected. Table S1 has now N=722 Entomobrya and $\mathrm{N}=428$ Lepidocyrtus.

L120-121, Here it says $N=98$ sites, but Table S1 has site ID\#'s to go up to 102. If might be worth adding to the Table S1 caption that site ID\#'s are not consecutive numbers?

We have added to table header: "Sites 46, 70, 71 and 82 are absent from the list as no specimens were collected at these siles"

L124-125, rephrase "posterior identification checks"

We think it is OK.

L130-131, Of the "100 $\mu \mathrm{L}$ volume of proteinase $\mathrm{K}+$ Buffer ATL", how much of each component / mixed in what ratio?

90 ul of Buffer ATL + 10 ul of proteinase $\mathrm{K}$ (ratio 9:1 which is the same ratio found in the original protocol).

L138, expand "PCR" on first mention (earlier)

This is expanded in line 144 and we do not see the need to expand it before.

L150-152, This needs to be shifted down, since the basic of unit of analysis is an OTU, yet the information on how they were delineated comes later in the 
paragraph.

This has been completely revised.

L152-154, It is not immediately clear why a NJ phylogenetic analysis was necessary, given that all that's needed to define OTUs is pairwise p-distances. If the tree is needed for some other purpose, then that should be stated.

This has been completely revised.

L155-158, What is the relevance here of choices relating to the OTU delineation threshold that was previously made by Cicconardi et al. 2013? Is there something biologically meaningful about this $96 \%$ similarity cut-off (e.g., corresponds with a natural division between intra- vs. inter-specific variation seen for named species), or this used just for consistency across studies? Either way, rationale should be given, including why the use of different genes (COI here vs. COIl in Cicconardi et al. 2013) would not be problematic for this purpose (there are indications in the following paragraph that $\mathrm{COI}$ mutation rate is quite a bit faster, almost $2 x$, than that of COII).

We have extensively discussed these points in previous answers to Referee 1. Please, check responses to topics "OTU delineation" and "Evolutionary rate estimation" in Referee 1.

L158-161, this last sentence indicates what was done (i.e., a BOLD comparison), but does not clearly explain the purpose of doing it.

We explained that it was done "for evidence of identical or nearly identical sequences sampled outside the Great Britain". 
L165 \& 167, I presume these rates are per lineage (cf. between a pair of diverging lineages), but it would good to explicitly state that this is the case.

These are general rates proposed for different groups within arthropods.

L192, Following mapping, how were non-random patterns identified?

Patterns were identified visually. Please, refer to our answer to similar question to Referee number 1.

L197 \& 201, there is some redundancy here re: the partitioning into glaciated vs. unglaciated groupings.

In these lines, we have explained how sampling sites were classified into glaciated and unglaciated areas to test for richness differences between these areas. We did not see the redundancy indicated.

L208-210, meaning unclear: "...differences in sampling and abundances..." and "...as a threshold the abundance of the smaller...". Is "abundance" the essentially just the sample size (i.e., number of sequenced individuals), or something other?

Yes, abundance essentially means sample size $=$ number of sequences per sampling sites

L212-213, An a priori hypothesis is given for OTU-level richness but no hypothesis is given from intra-OTU richness, and so this seems incomplete.

This has been changed to include intra-OTU diversity.

L214-215, the reference to "communities" here (i.e., smaller, or rarefied) is confusing. I think it just intended to refer to glaciated vs. unglaciated groupings, but it doesn't give the same clarity (i.e., does not convey which of 
this two had the small sample size). Also, the idea that a community (in the ecological sense) would be comprised only of members of two Collembola genera seems to be a narrow view.

Yes, we just intend to refer to the groupings found in each area. The term community comes from the software used. We have added an explanation of what we mean by community in lines 262-263 and Tables 3 and 4.

\begin{abstract}
RESULTS
L221-231, some persistent issues with correspondence between sample sizes reported in the Methods, here in the Results, and in Table S1. Perhaps a clearer distinction needs to made between number of specimens collected and assessed on the basis of morphology vs. number of collected specimens use for molecular analyses.
\end{abstract}

Table S1 (now Table S1.1 in Appendix S1) has been amended and it now includes all specimens collected and assessed on basis of morphology. All specimens were sequenced, however, some of them failed to amplify or produce sequences of good quality traces (as explained in lines 285-286). This is why numbers differ.

L235, reword: "...clustering sequences [into] OTUs..."

We think [down to] is OK.

L241 (\& 258): What is the importance of OTUs that closely matched sequences in BOLD? It is not clear to me why these are being highlighted. 
Identical or near identical BOLD matches are highly indicative that the sequences belong to the same species, and their geographic origin can be informative about introduction (Cicconardi et al., 2017).

L252, typo [OUT]

Changed.

L267, replace "well" with "broadly" (or similar).

Replaced.

L269, delete word [more]

Deleted.

L269-275, The conclusion of non-random geographic structuring / clear structuring of genetic variation within OTU 4 is subjective, and perhaps questionable. From looking at Fig. 4, the two subgroups (NivG1 and NivG2) are broadly sympatric at 3 sampling sites and allopatric at 4 sites, so the presence / absence of substructure could be seen as somewhat equivocal.

Rather than subjectively, we based our conclusion on the high bootstrap support value found for these groups in the NJ analysis, which has been corroborated by the high posterior value found in the Bayesian inference and inspection of their geographic distribution. Both suggested the existence of these two monophyletic clusters within OTU4. The fact that they are broadly sympatric does not change conclusions. 
Also, from Figure 2, inclusion of haplotype C019 in the subgroup NIVG1 is odd, and is inconsistent with the reported node support value (bs=67\%) for the monophyly of this group.

This was an error when shading the subgroup, C019 does not belong to NIVG1

L278, rephrase "presented spread/widespread"

We believe it doesn't need to be rephrased.

L280, replace "unique" with "different" (or similar).

\section{Changed to single.}

L281-282, comment: the labelling structure of OTUs is confusing. For Entomobrya (and most Lepidocyrtus) spp., each different OTU (also sometimes referred to as "lineage") is identified by a different integer. However, in a few cases for Lepidocyrtus spp., different OTUs are identified by a decimal place (e.g., OTU 7.1 and OTU 7.2), and this also means that "lineage" is no longer synonymous with "OTU" (e.g., lineage 7 is a higher-level group). I think that the inconsistency introduced by the latter should be avoided. Also, the decision to pool different OTUs together (e.g., OTU $7.1+$ OTU 7.2 = OTU 7) causes the earlier definition of what an OTU is (i.e., the $4 \%$ rule) to now be inaccurate. It is also unclear if this treatment was applied prior to conducting richness analyses, or afterwards. I think it would be better to avoid this reclassification altogether.

This has been completely revised after phylogenetic inferences.

L287, replace "disjunctive" with "disjunct" 
Both terms are correct.

L295-297, as above, that conclusion that there is geographic structuring of genetic variation is subjective (e.g., no test was performed). In this case, there is also no opportunity for the reader to see for themself what the authors are refer to, given that subgroups within OTUs 3, 7, 8, 12 and 18 are not identified on Figure 3, and their spatial distributions are not mapped in Figure 5.

There are no subgroups within these OTUs, they are themselves whole OTUs that show geographic structuring of genetic variation and their distributions are indeed indicated in Figure 3 and mapped in Figure 5.

L311, "time" (rather than "times")

Times is correct because it does not refer to chronology but to the number of times a random sample is drawn from a community

L315, replace word: "communities" (within-OTU was previously described as equivalent to within-species).

The word "communities" has been clarified, as discussed above, and we have refrained from using species terminology.

L325, "lower" (rather than "smaller")

We rather stick to smaller because it clearly denotes something is reduced.

This fits better to the reduced diversity found than the word lower, which relates more to a position, e.g. subordinate, inferior, worse.

L327 \& 328, the level of organization here is among individuals / populations within species (not within communities). 
As explained above, the use of the term community has been explained in line 262-263.

L333, the best model applies to a set of sequences, not to groups

Changed to "for sequences in the two Entomobrya monophyletic groups"

L334-337, as noted above, inclusion of haplotype C019 in the subgroup NIVG1 is odd. Also, something that is not mentioned here or apparent from Figure 4 is which of the two subgroups (i.e., NivG1 or NivG2) has a geographic distribution spanning the glaciated and unglaciated areas.

CO19 has been incorrectly included in NIG1 and this has been amended in Figure 2. Their geographic distribution has been mentioned in lines 341-342 and we added how they span through glaciated and unglaciated areas in lines 342-343.

DISCUSSION

L350, "confined" (rather than "constrained")

Changed to "restricted".

L356, "characteristics" (rather than "arguments")

We are talking about arguments to support a hypothesis, thus, we believe the word is appropriate.

L359-364, From the shading of these subgroups on Figure 2, it appears that NivG1 includes 11 (not 10) individuals, and NivG2 includes 7 (not 6) individuals. 
We re-built the tree and redraw subgroups making sure the right individuals have been shaded.

L373, "long-distance" (rather than "long-term")

Changed.

L381, missing word: "...origin in [the] Great Basin..."

This article is not needed before the name of the country (Great Britain).

L385-385, The meaning of "leading front" are "pre-empt space" are both somewhat unclear.

The ideas conveyed by both terms are commonly discussed in the literature concerning genetic consequences of Pleistocene glaciations.

"Leading front" ( leading edge) is a common term used to explain patterns of colonization after ice sheets retreat [(e.g. "dispersal at the leading edge would likely be by long distance dispersants that set up colonies far ahead of the main population" in Hewitt (1999)]. "Pre-empt space" also relates to the dynamics of post-glacial recolonization where empty spaces resultant from ice sheet advance, are now filled by pioneering dispersants [( e.g. "these pioneers could expand rapidly to fill the area before significant numbers of other dispersants arrived' in Hewitt (1999)].

L394, Until here, it was not apparent that morphospecies diagnosis is based on color pattern characters. I think it is important to mention in the Methods (around L123), since this would probably affect readers' expectations about the extent to which such diagnosis would match DNA-based OTUs.

It has been added to Methods, lines 129-130.

L395, this should refer to Figure 2 (not Figure 1) 


\section{Changed.}

L339, replace "occupied" with "contained"

Changed to "featured in".

L402-403, this information on key taxonomic features should be mentioned in the Methods.

Done.

$L 409$, There is no reason to expect that whole genera would respond to environmental change as single cohesive unit.

We agree, and that is not what we are suggesting. We merely point of there is a difference between genera.

L410 (and elsewhere), replace "presented" with another word.

We believe this word is OK.

L413-415, First it is stated that the Lepidocyrtus OTU richness data do support the "above" hypothesis (the only one mentioned by name was the northern poverty hypothesis), but then it is state that the geographic distribution of Lepidocyrtus species richness adds further support for the longterm persistence. The switch between OTU and species is also a source of confusion, since the previous paragraph talks about species in the strict sense of morphospecies... it is not clear if that carries over to this paragraph.

The word species has been changed to OTU throughout the text as explained before. 
Sentence mentioning long-term persistence has been deleted to avoid confusion and this same discussion is presented in the following paragraph.

L419, "population persistence of [species]" seems a bit awkward.

Word order changed.

L420, delete word: [some]

We prefer to keep this word because it denotes that this is a relative rather than absolute age estimative.

L425-426, But note that the lower bound of the HPD surrounding the point estimate of 53 Kya does not predate the LGM.

As explained in a previous response to referee 1, this analysis has been revised by changing the tree prior used. Ages have changed and lower bounds of HPD do predate LGM for both NVG1 and NIVG2.

\section{TABLES}

Table 1: I think that L661 of the caption should be "number of [haplotypes] sampled from single localities", and that L662 would be clearer if it read as "observed [intra-OTU] p-distances". As noted above, I am not really sure what value the BOLD comparison adds. Also, there is no mention of what the asterisks in the "morphospecies" column indicate.

These are in fact number of sequences and not number of haplotypes sampled from single localities. 
The observed [intra-OTU] p-distances are reported as mean p-distances and we also think it is good to present maximum $p$-distances as it gives a more complete idea of their variation.

As explained in the caption, BOLD information refers to the localities from where identical or near identical sequences (>99\% similarity) were sampled which gives an idea of their restricted (no match to the database) or worldwide distribution (e.g. Canada, Australia, etc.)

We did mention what asterisks indicate just below the table but superscript alphabetic letters have substituted them.

Table 2: Unclear why the structure of this table differs from Table 1 by not listing "number of [haplotypes] sampled from single localities". In the BOLD column, what do "no match", "not provided" and "<99\% match" mean? None of these designations appear in Table 1.

Tables have now similar structure. "No match" means that when querying a sequence into Bold database, no hit was found, i.e., there is nothing like this sequence already published in the database. "Not provided" means, although, a similar sequence was found in the database, the geographic information of the place where this sequence was sampled from is missing. "<99\% match" means that, although, similar sequences were found, they are less than $99 \%$ identical which preclude us to consider it for taxonomic or geographic assignments.

Table 3: The word "community" on L694 does not apply broadly across this table and should be replaced. Also, it is unclear why many of the mean and $95 \% \mathrm{Cl}$ values are not given (instead reported as a dash).

We have added a sentence to the Table caption to explain what we mean by community. Mean and $\mathrm{Cl}$ can only be given for the community that is being 
rarefied for each comparison, this is why a dash sign appears in the other community.

Table 4: see comments on Table 3.

Changed.

Table 5: it is unclear why the corresponding intra-OTU MRCA \& Cl's for Entomobrya spp. are not included here.

This result is presented in the text, lines 409-11, because there were only 2 monophyletic clusters for Entomobrya, not enough to build a table. Table 5 only deals with Lepidocyrtus results.

Table S2: The purpose of summarizing data from a different gene (COII), from a different location (Panama data), from a different study (add citation) should be given here. Also abbreviated table headings need to be expanded / defined in the caption.

Information added.

\section{FIGURES}

Figure 1. An inset showing the broader geographic context would be a useful addition.

Our study focuses on the Great Britain geographic context, and differs from many other studies that look at a wider European context. Thus, we believe that the figure would not benefit from widening its view and we rather keep it to GB to avoid any confusion.

Figure 2. The inclusion of haplotype C019 in the subgroup NIVG1 within lineage 4 (= OTU 4 ) is odd. If poorly supported monophyly is not seen to be 
problematic when delimiting subgroups, then other sequences (i.e., nivalis $L ; 20,23$ etc) should also be included in NIVG1. Alternatively, if at least moderately supported monophyly is a requirement, then haplotype C019 should be exclude from subgroup NIVG1 (and its geographic distribution excluded from the ellipse in Fig. 4).

This was an error when shading the group. C019 is not part of NIVG1. This figure has been completely revised.

Figure 3. As noted above, the shading of OTUs 3, 7, 8, 12 and 18 (i.e., those in which there is reported to be geographic structuring of genetic variation) is not all that meaningful if subgroups within these OTUs are not identified and named, and also represented on Figure 5

There are no subgroups within these OTUs. The entire OTU itself is a monophyletic cluster. This differs from Entomobrya data in which we found monophyletic clusters within a widespread OTU (number 4). No monophyletic clusters were found within Lepidocyrtus widespread lineages. This difference is explained in the text, lines 361-364.

Figure 4. The caption / figure does not clarify which ellipse represents which subgroup in panel $B$.

Caption changed accordingly.

Figure 5: see comment on Figure 3.

Please, see response to comment on Figure 3.

References

Baddeley, A. \& Turner, R. (2005) spatstat: An R Package for Analyzing Spatial Point Patterns. Journal of Statistical Software, 12, 1-42. 
Brochmann, C., Gabrielsen, T.M., Nordal, I., Landvik, J.Y., \& Elven, R. (2003) Glacial survival or tabula rasa? The history of North Atlantic biota revisited. Taxon, 52, 417-450.

Burkhardt, U. \& Filser, J. (2005) Molecular evidence for a fourth species within the Isotoma viridis group (Insecta, Collembola). Zoologica Scripta, 34, 177-185.

Cicconardi, F., Borges, P.A.V., Strasberg, D., Oromí, P., López, H., PérezDelgado, A.J., Casquet, J., Caujapé-Castells, J., Fernández-Palacios, J.M., Thébaud, C., \& Emerson, B.C. (2017) MtDNA metagenomics reveals large-scale invasion of belowground arthropod communities by introduced species. Molecular Ecology, 26, 3104-3115.

Cicconardi, F., Fanciulli, P.P., \& Emerson, B.C. (2013) Collembola, the biological species concept and the underestimation of global species richness. Molecular Ecology, 22, 5382-5396.

Cicconardi, F., Nardi, F., Emerson, B.C., Frati, F., \& Fanciulli, P.P. (2010) Deep phylogeographic divisions and long-term persistence of forest invertebrates (Hexapoda: Collembola) in the North-Western Mediterranean basin. Molecular Ecology, 19, 386-400.

Dixon, P.M. (2002) Ripley's K function. Encyclopedia of environmetrics (ed. by A.H. El-Shaarawi and W.W. Piegorsch), Wiley, Chichester New York.

Ezard, T., Fujisawa, T., \& Barraclough, T.G. (2009) SPLITS: SPecies' LImits by Threshold Statistics: R package version 1.0-18/r45.

Funk, D.J. \& Omland, K.E. (2003) Species-level paraphyly and polyphyly: Frequency, causes, and consequences, with insights from animal mitochondrial DNA. Annual Review of Ecology Evolution and Systematics, 34, 397-423.

Garrick, R.C., Rowell, D.M., Simmons, C.S., Hillis, D.M., \& Sunnucks, P. (2008) Fine-scale phylogeographic congruence despite demographic 
incongruence in two low-mobility saproxylic springtails. Evolution, 62, 1103-1118.

Garrick, R.C., Sands, C.J., Rowell, D.M., Hillis, D.M., \& Sunnucks, P. (2007) Catchments catch all: long-term population history of a giant springtail from the southeast Australian highlands - a multigene approach. Molecular Ecology, 16, 1865-1882.

Hewitt, G.M. (1999) Post-glacial re-colonization of European biota. Biological Journal of the Linnean Society, 68, 87-112.

Hopkin, S.P. (2007) A Key to the Collembola (springtails) of Britain and Ireland. Field Studies Council (FSC) AIDGAP, Shrewsbury, UK.

Kapli, P., Lutteropp, S., Zhang, J., Kobert, K., Pavlidis, P., Stamatakis, A., \& Flouri, T. (2017) Multi-rate Poisson Tree Processes for single-locus species delimitation under Maximum Likelihood and Markov Chain Monte Carlo. Bioinformatics, 33, 1630-1638.

Kembel, S.W., Cowan, P.D., Helmus, M.R., Cornwell, W.K., Morlon, H., Ackerly, D.D., Blomberg, S.P., \& Webb, C.O. (2010) Picante: R tools for integrating phylogenies and ecology. Bioinformatics, 26, 14631464.

Porco, D., Bedos, A., Greenslade, P., Janion, C., Skarżyński, D., Stevens, M.I., Jansen van Vuuren, B., \& Deharveng, L. (2012) Challenging species delimitation in Collembola: cryptic diversity among common springtails unveiled by DNA barcoding. Invertebrate Systematics, 26, 470-477.

Skarzyński, D. (2004). Taxonomic status of Schaefferia emucronata Absolon, 1900 and Schaefferia willemi (Bonet. 1930) (Collembola, Hypogastruridae) in the light of hybridisation studies. Deutsche Entomologische Zeitschrift, 51, 81-86. 
South, A. (1961) The taxonomy of the British species of Entomobrya (Collembola). Transactions of the Royal Entomological Society of London, 113, 387-416.

Stamatakis, A., Hoover, P., \& Rougemont, J. (2008) A rapid bootstrap algorithm for the RAxML Web servers. Systematic Biology, 57, 758771.

Swenson, N. (2014) Functional and Phylogenetic Ecology in R. SpringerVerlag, New York.

Tzedakis, P.C., Emerson, B.C., \& Hewitt, G.M. (2013) Cryptic or mystic? Glacial tree refugia in northern Europe. Trends in Ecology \& Evolution, 28, 696-704.

Zhang, J., Kapli, P., Pavlidis, P., \& Stamatakis, A. (2013) A general species delimitation method with applications to phylogenetic placements. Bioinformatics, 29, 2869-2876. 
3 Christiana M. A. Faria ${ }^{1 *}$, Peter Shaw $^{2}$, Brent C. Emerson ${ }^{3}$

4

${ }^{1}$ Centre for Ecology, Evolution and Conservation, School of Biological Sciences, University of East Anglia, Norwich Research Park, Norwich, NR4 7TJ, Great Britain

${ }^{2}$ Centre for Research in Ecology, Whitelands College, Holybourne Ave., London SW15 4JD, Great Britain

${ }^{3}$ Island Ecology and Evolution Research Group, Instituto de Productos Naturales y Agrobiología (IPNA-CSIC), c/Astrofísico Francisco Sánchez 3, La Laguna, Tenerife, Canary Islands, 38206, Spain

*Current address: Laboratory of Ecology and Evolution of Insects, Department of Biology, Centre for Sciences, Building 906, Federal University of Ceará, 60455970, Fortaleza, CE, Brazil

Corresponding author: Christiana M. A. Faria, Address: Laboratory of Ecology and

Evolution of Insects, Department of Biology, Centre for Sciences, Building 906, Federal

University of Ceará, 60455-970, Fortaleza, CE, Brazil, Fax number: +558533669806 ,

21 Email address: fariacma@gmail.com

22

23 Running title: Pleistocene persistence of Collembola in Great Britain 


\section{ABSTRACT}

32

33 Aim Using two genera of Collembola, Lepidocyrtus and Entomobrya, we test for 34 genetic signatures of Pleistocene persistence of soil arthropods in Great Britain.

35 Location Great Britain.

36 Methods A region of the mitochondrial COI gene was sequenced for 1150 Collembola 37 specimens from the genera Lepidocyrtus and Entomobrya across Great Britain. 38 Individuals were clustered into OTUs, and both OTU richness and geographic patterns 39 of genetic variation within OTUs were compared between glaciated and unglaciated 40 areas to identify signatures of OTU persistence through Pleistocene glacial events.

41 Results Our analyses identified 12 Entomobrya and 18 Lepidocyrtus OTUs in Great 42 Britain. Lepidocyrtus OTU richness was significantly lower in glaciated than 43 unglaciated areas, whereas there was no difference for Entomobrya OTU richness. 44 However, both genera presented clear patterns of geographically disjunct genetic 45 variation and geographically localized diversification of OTUs. Estimated dates for the 46 onset of in situ diversification events indicate population persistence that precede the 47 last glacial maximum.

48 Main conclusions. Patterns of genetic diversity within Collembola OTUs in Great 49 Britain add to a growing body of evidence that elements of the invertebrate fauna have 50 persisted in situ through Pleistocene glacial cycles. Genetic signatures of population 51 persistence in more northern glaciated areas of Great Britain support a hypothesis of 52 geothermal glacial refugia that call for further investigation with other soil mesofaunal 53 taxa.

54 Key-words: Collembola, mesofauna, Pleistocene, refugia, soil, western Europe 


\section{INTRODUCTION}

56

57 Climate oscillations have considerably affected the distributions of many species 58 globally, resulting in the north-south structuring of genetic diversity within many 59 species as a result of past range contraction and expansion (e.g. Hewitt, 2004). In the 60 northern hemisphere, during the Pleistocene glaciations (from 2.59-0.012 Million years 61 ago, Ma), as high latitudes were covered by ice and permafrost, northern populations 62 either went extinct or their distributions retreated southward, surviving in refugia 63 (Tzedakis et al., 2013). Due to their geographic position and latitudinal range, large 64 areas of the British Isles were repeatedly glaciated throughout this period (Chiverrell \& 65 Thomas, 2010), resulting in a present-day biota of reduced diversity, limited endemism, 66 and typically recent origin from continental Europe (Yalden, 1982; Searle, 2008; 67 Montgomery et al., 2014).

While clearly an appropriate model for many species, the generality of this view has

70 been challenged by evidence that several species persisted through glaciations in some 71 areas of Ireland (Teacher et al., 2009), the English Channel (Coyer et al., 2003; Provan et al., 2005; Hoarau et al., 2007) and more recently in the Great Britain (McInerney et

73 al., 2014). Evidence has recently been presented for an ancient groundwater fauna 74 endemic to Britain and Ireland, of which two amphipod species from the genus 75 Niphargus were estimated to have persisted for at least 19.5 million years (McInerney et 76 al., 2014). The survival of groundwater dwelling organisms was probably facilitated by 77 the environmental stability of hypogean environments compared to the surface water 78 (i.e. water temperature and chemistry buffering) (Gibert et al., 1994; McInerney et al., 
79 2014). With regard to the terrestrial environment, the only convincing evidence for

80 Pleistocene persistence comes from the frog, Rana temporaria, which shows several 81 related mitochondrial DNA cytochrome $b$ haplotypes that are endemic to Ireland 82 (Teacher et al. 2009).

83

84 Signatures of population persistence, such as that presented by Rana temporaria, can 85 provide compelling evidence for Pleistocene persistence in the form of in situ patterns 86 of allelic divergence and/or gradients of haplotype richness between unglaciated and 87 glaciated areas. However, these signatures may be obscured for more dispersive species 88 by subsequent gene flow. During the Last Glacial Maximum (LGM), the British ice 89 sheet achieved its maximum extent at different times (between 27 and 15 thousand 90 years ago, ka) in different sectors (Clark et al., 2012, see Fig. 15), but left ice-free 91 habitats, particularly in the south, that could have acted as refugia. Under this model, the 92 persistence of southern British refugial populations would facilitate local accumulation 93 of diversity and subsequent "southern richness northern poverty" of genetic variation as 94 temperatures rise, ice sheets retract, and populations expand northwards, as proposed for 95 pan-European species (Hewitt, 2000).

97 Sedentary soil invertebrates like Collembola have been shown to survive harsh 98 conditions, such as Pleistocene climate change, and retain genetic signatures in the form 99 of geographically structured genetic variation (e.g. Stevens et al., 2007, Garrick et al., 100 2008, Cicconardi et al., 2010). Their wingless morphology limits dispersal ability (and 101 hence gene flow), while their high local abundance (10,000 $\mathrm{m}^{-2}$ upwards, Hopkin, 1997) 102 increases survival probability relative to other less abundant arthropod groups (Emerson 
103 et al., 2011).

104

105 Here we sample two widespread Collembola genera across Great Britain, Entomobrya 106 and Lepidocyrtus, to test for the following signatures of population persistence prior to 107 the last glaciation: (i) lower OTU richness and lower intra-OTU genetic richness in 108 northern glaciated areas compared to southern unglaciated areas; (ii) local monophyly 109 (e.g. geographically restricted monophyletic groups of sequences); (iii) coalescent 110 origins for endemic genetic variation that predates the LGM; (iv) disjunct distributions 111 of OTUs that cannot be explained by long distance dispersal. We evaluate evidence for 112 these expectations by sampling the mitochondrial DNA COI gene for 722 specimens of 113 Entomobrya and 428 specimens of Lepidocyrtus sampled from across Great Britain.

\section{MATERIAL AND METHODS}

\section{Sampling and laboratory work}

119 Samples were collected from 98 sites across Britain between 2011 and 2012, aiming for 120 approximately equal sampling effort across the glaciated and unglaciated areas of 121 England and Wales, collecting particularly from the geographical extremes of the region 122 (Fig. 1, see Supporting Information Appendix S1: Table S1.1 for details). At each 123 location, surface-active animals were collected by vacuum collection (two sets of 30 124 second collections, transferred directly to EtOH), and duplicate soil / litter samples were 125 collected with minimal disturbance into plastic bags for subsequent extraction with a 126 Tullgren funnel system followed by manual removal of the relevant genera. Upon 
127 sorting, samples were individually placed in 96-well PCR plates and stored into

128 absolute ethanol at $4^{\circ} \mathrm{C}$ prior to identification by PS. Identification to morphospecies 129 followed Hopkin (2007) and body scales and colour-pattern were mainly used as key 130 characters for diagnosis. At least 1 photo per morphospecies per site was taken to help 131 posterior identification checks.

132

133 DNA was isolated using the DNeasy 96 well Blood and Tissue Extraction Kit 134 (QIAGEN, West Sussex, Great Britain), following manufacturer's instructions with the 135 following modifications to preserve the exoskeleton of each animal. After removing 136 ethanol from the PCR collection plates, digestion was performed by adding a $100 \mu \mathrm{L}$ 137 volume of proteinase $\mathrm{K}+$ Buffer ATL working solution (1:9 ratio). Samples were 138 incubated for just one hour at $56^{\circ} \mathrm{C}$ to avoid skeleton lysis. After incubation, lysates 139 were transferred to the DNeasy 96 collection tubes and the plates with remaining 140 exoskeletons were refilled with absolute ethanol and stored as vouchers at $4^{\circ} \mathrm{C}$.

142 DNA extracts were amplified for the 658 bp barcode region with the primers ColFol-for 143 (modified from primer LCO1490) and ColFol-rev (modified from primer HCO2198) 144 (Ramirez-González et al., 2013). Polymerase chain reactions (PCR) contained NH4 145 buffer (1x), $3.0 \mathrm{mM} \mathrm{MgCl}_{2}, 2.5 \mathrm{mM}$ of each dNTP, $0.4 \mu \mathrm{M}$ of each primer and $0.5 \mathrm{U}$ of 146 Taq polymerase (Bioline) in $25 \mu \mathrm{L}$ final volume and used the following thermal profile: $14795^{\circ} \mathrm{C}$ for $2 \mathrm{~min}, 40$ cycles of $95^{\circ} \mathrm{C}$ for $1 \mathrm{~min} ; 52^{\circ} \mathrm{C}$ for $45 \mathrm{~s}, 72^{\circ} \mathrm{C}$ for $1 \mathrm{~min}$; and finally $14872^{\circ} \mathrm{C}$ for $5 \mathrm{~min}$. PCR products were cleaned with ExoSap, then normalized to a 149 concentration of $5 \mathrm{ng} / \mu \mathrm{L}$ and sent to Eurofins for sequencing with the reverse primer. 


\section{Sequence alignment and molecular OTU delineation}

152

153 MtDNA COI sequences were processed with manual assessment of ambiguous base

154 calls with GENEIOUS PRO v.5.6.4 (Kearse et al., 2012) and then aligned using MAFFT

155 v.6.814 (Katoh et al., 2002). In order to define molecular OTUs, we explored Bayesian

156 Inference (BI) and Maximum likelihood (ML) approaches to build trees and different

157 delimitation methods (GMYC, mPTP and a clustering threshold of 96\% similarity) to

158 account for their inherent limitations (Luo et al., 2018). First, all sequences for each

159 genus were used to infer ML trees using maximum likelihood in RAxML (Stamatakis et

160 al., 2008) with a GTR+G substitution model (4 categories). We used the webserver

161 (https://raxml-ng.vital-it.ch/\#/) and ran full analyses with automatic bootstrap replicates.

162 After removing the outgroup sequence, inferred ML trees were then used for OTU

163 delimitation by mPTP analysis (Kapli et al., 2017) using the webserver (https://mptp.h-

164 its.org/\#/tree) with the multi rate poisson tree processes method. Second, we obtained

165 ultrametric BI trees running BEAST v.1.8.4 (Drummond et al., 2012) using all sequences

166 for each genus, a GTR $+\mathrm{G}$ (4 categories) model, and a combination of Tree and Clock

167 priors using the Cipres Science gateway (https://www.phylo.org/portal2). However,

168 BEAST output estimates were problematic due to convergence issues, particularly for

169 clock.rate. This is likely due to the nature of the dataset, which has both speciation and

170 coalescent signals that cannot be fully resolved with either a Yule or Coalescent tree

171 prior. Thus we refrained from using BI trees representing all haplotypes for GMYC

172 approach (Generalized Mixed Yule Coalescent, Fujisawa \& Barraclough, 2013). As an

173 alternative, we used DNAcollapser (Villesen, 2007) to reduce dataset to unique

174 haplotypes and re-ran BEAST with GTR $+\mathrm{G}$ (4 categories), Yule tree prior and clock 
175 prior fixed to 1 . Two independent runs of $5 \times 10^{7}$ Markov Chain Monte Carlo (MCMC)

176 generations were sampled every 1000 generations. Runs were well converged and ESS

177 values were $>200$. BI ultrametric trees were then used as input files for GMYC in $\mathrm{R}$

178 using 'splits' package (Ezard et al., 2009). ML trees were also inferred with the unique

179 haplotypes dataset and mPTP analysis repeated for both ML haplotype and BI haplotype

180 trees. Third, we used a 96\% similarity threshold for clustering of Entomobrya and

181 Lepidocyrtus sequences into OTUs. This threshold is based on prior data available from

182 several studies that have extensively investigated the temporal-spatial aspects and

183 biological significance of cryptic molecular lineage diversity in the genus Lepidocyrtus,

184 one of the two genera investigated in the present study (Cicconardi et al., 2010, 2013).

185 Following phylogenetic reconstruction, GMYC analysis, Hardy-Weinberg equilibrium

186 and linkage-disequilibrium tests, 19 molecular lineages occurring sympatrically were

187 found to be congruent with biological species (Cicconardi et al. 2013). We then used

188 sequences from these 19 molecular lineages to calculate their pairwise distances and

189 selected the minimum p-distance found between them (0.04) to define our clustering

190 threshold (96\%) (Appendix S1: Table S1.2). Once OTUs were defined, one sequence

191 per OTU was submitted to the BOLD Identification System for evidence of identical or

192 nearly identical sequences sampled outside the Great Britain.

193

194 Evolutionary rate estimation and dating analysis

195

196 General mutation rates ranging from $0.006-0.0248 \%$ substitutions/site/Ma (million

197 years ago) have been proposed for the mtDNA genome of insects, mostly for the COI

198 gene (e.g. DeSalle et al. 1987, Brower 1994, Wares 2001, Ho \& Lo 2010, and 
199 references therein). Following an extensive comparative rate analysis using COII 200 sequences from across Pancrustacean major lineages, a conservative (fast) COII mean 201 rate of 0.0245 substitutions/site/Ma has been estimated for the genus Lepidocyrtus 202 (Cicconardi et al. 2010). However, currently there is no COI external rate available for 203 Collembola. Thus, we applied this fast COII evolutionary rate to a set of corresponding 204 sequences to estimate a COI rate as follows. First, the root height of eight Lepidocyrtus 205 COII sequences sampled sympatrically in Panama (Cicconardi et al., 2013) was 206 obtained running BEAST using the COII rate of Cicconardi et al. (2010). This root height 207 was then applied to a corresponding COI sequence dataset for the same specimens 208 (Emerson et al., 2011) to estimate the rate of substitution of the COI gene. Tamura-Nei 209 plus Gamma (TN93+G) and Tamura 3-parameter plus Gamma (T92+G) were selected 210 as the best model for the COI and COII data respectively in MEGA v.6.06 (Tamura et al., 211 2013). The BEAST estimated root age of the eight Panamian Lepidocyrtus COII 212 sequences was $35 \mathrm{Ma}(\mathrm{HPD}=12.8-60 \mathrm{Ma})$. When this root age was applied to the 213 corresponding COI sequences, an estimated substitution rate of 0.0421 214 substitutions/site/Ma for the COI gene was obtained with BEAST. As the COII rate 215 estimate of Cicconardi et al. (2010) is considered an overestimate of the true rate, 216 yielding probable underestimates of divergence times (Cicconardi et al. 2010), 217 divergence times derived from our COI rate are also likely to be underestimates.

219 The COI rate was then used in BEAST to estimate the time of the most recent common 220 ancestor (MRCA) for nodes of interest within the Entomobrya and Lepidocyrtus BI 221 trees, i.e. dating analysis applied separately only for the geographic localized 222 monophyletic clusters. The most appropriate substitution model for each dataset was 
223 estimated using MEGA. An uncorrelated lognormal relaxed clock (with clock.rate priors

224 set to ucdl.mean $=0.042$, ucdl.std $=0.11$ ) and a coalescent tree prior (intraspecific 225 dataset) were applied to two independent runs of $10^{7}$ Markov Chain Monte Carlo 226 (MCMC) generations, sampled every 1000 generations. Convergence among the 227 independent runs, and expected sampling sizes (ESS) for the posterior distribution and 228 parameters were evaluated using TRACER v.1.6 (Rambaut et al., 2014).

\section{Geographical distribution and OTU diversity analysis}

232 Haplotype distributions were mapped using QGIS (QGIS Development Team, 2014), to

233 evaluate non-random patterns of (i) geographically localised monophyletic clades of 234 mtDNA sequences, consistent with population persistence over an evolutionary 235 (mutational) timescale, and (ii) geographically disjunct distributions of OTUs, 236 consistent with range fragmentation. While these distributions were visually inspected, 237 we also used Ripley's K function (Kdot function) to test whether these clades are 238 dispersed, clustered or randomly distributed throughout the study area (Dixon, 2002). 239 Kdot was tested for each OTU of interest (marked point pattern) in R using package 240 'spatstat' (Baddeley \& Turner, 2005). Three corrections $\left(\mathrm{K}_{\mathrm{iso}}-\right.$ isotropic, $\mathrm{K}_{\text {trans }}-$ 241 translation correction, $\mathrm{K}_{\mathrm{bord}}$ - border method) were applied to observed data while 242 estimating $\mathrm{K}$ function and these were compared to what it would be expected if spatial 243 distributions were random ( $\mathrm{K}_{\text {pois }}$ - completely random Poisson point process).

245 To test for diversity richness differences between glaciated and unglaciated areas, we 246 used permutation analyses in ECOSIM v.7.72 (Gotelli \& Entsminger, 2000) to analyse 
247 matrices of (i) OTU richness within sampling sites and (ii) intra-OTU haplotype 248 richness within sampling sites. Sampling sites were classified into two biogeographical 249 areas: glaciated and unglaciated, defined according to the maximum limits of the 250 British-Irish ice sheet (BIIS) during the last Pleistocene glacial period (Clark et al., 251 2012). This represents the most up-to-date reconstruction of the extent and times of 252 BIIS, derived from evidence such as moraines, lateral meltwater channels, and 253 subglacial melt. Although it reached its maximum extent at different times in different 254 sectors (see Fig. 15 in Clark et al., 2012), its overall extent, independent of the time, was 255 used to classify each locality as either glaciated or unglaciated (Fig. 1). We used the 256 rarefaction algorithm implemented in ECOSIM to control for differences in sampling and 257 abundances when testing for OTU and intra-OTU richness differences, using as a 258 threshold the abundance of the smaller community being compared. The random 259 number seed was set to 10 and simulations were run for 1000 iterations. Richness 260 curves and their 95\% confidence intervals were generated and hypotheses of equivalent 261 OTU richness and intra-OTU haplotype richness for glaciated and unglaciated 262 communities (i.e. collection of Entomobrya haplotypes and Lepidocyrtus haplotypes 263 found in each area) was evaluated by assessing whether the observed richness of the 264 smaller community fell within the $95 \%$ confidence interval of the simulations for the 265 rarefied community.

267 RESULTS 
271 We collected a total of 1150 individuals, 722 from the genus Entomobrya and 428 from

272 the genus Lepidocyrtus. Entomobrya specimens were sampled across 90 sampling sites

273 (Appendix S1), and were assigned to 6 morphospecies (Entomobrya albocincta, E.

274 intermedia, E. marginata, E. multifasciata, E. nicoleti and E. nivalis). Two Katianna sp.

275 sequences were sampled as outgroups. Five morphospecies were sampled from many

276 locations while the scarcer E. marginata was found in only a few sites (Table 1).

277 Lepidocyrtus specimens were sampled from 68 sampling sites (Appendix S1: Table

278 S1.1), and were assigned to 4 common morphospecies (Lepidocyrtus curvicollis, L.

279 cyaneus, L. lanuginosus, L. lignorum), two unknown morphospecies (Lepidocyrtus sp. 1

280 cf. orange, Lepidocyrtus sp. 2) plus one specimen of each of L. ruber and L. violaceus

281 (Table 2).

282

283 MtDNA COI sequences were obtained from 659 Entomobrya and 391 Lepidocyrtus

284 specimens, with alignment lengths of 561 and 556 bp respectively, and with 173 and 92

285 unique haplotypes respectively. The remaining specimens of each genus either failed to

286 amplify or produce sequences of good quality. Inferred ML trees using all haplotype

287 datasets for both genera are presented in Appendix S2. Using the multi coalescent rate,

288 mPTP delimited 12 Entomobrya OTUs and 17 Lepidocyrtus OTUs. Inferred ultrametric

289 BI trees using the unique haplotypes dataset for both genera are presented in Figures 2

290 and 3. A comparison of monophyletic clusters recovered by each tree inference methods

291 and their respective branch support is provided in Appendix S2. GMYC analysis using

292 BI ultrametric trees defined 22 ML entities for Entomobrya and 18 ML entities for

293 Lepidocyrtus. mPTP using BI haplotype trees delimited 13 Entomobrya and 22

294 Lepidocyrtus OTUs while for ML haplotype trees, mPTP delimited 12 Entomobrya and 
29518 Lepidocyrtus OTUs. Finally, using the $96 \%$ similarity threshold for clustering 296 sequences down to OTUs (see methods for details), a total of 13 Entomobrya OTUs and 29722 Lepidocyrtus OTUs were identified. OTU numbers and splits defined by each 298 method used are presented in Appendix S2.

299

300 Due to the slightly different number of OTUs defined by different methods, we have 301 taken a conservative approach of considering OTUs those that are recovered in the 302 majority of approaches employed and avoiding intra splits (Appendix S2: Table S2.2). 303 Thus, final numbers of OTUs are 12 for Entomobrya and 18 for Lepidocyrtus. 304

305 Of the 12 Entomobrya OTUs, two were singletons (Fig. 2). Overall mean divergence 306 among OTUs was 16\% (Table 1). Sequences assigned to the morphospecies $E$. 307 albocincta corresponded to a single OTU, while the other five morphospecies were 308 composed of two or three OTUs (Table 1, Fig. 2). The number of haplotypes per OTU 309 ranged from 1 to 44 (Table 1). The geographic distribution of the nine OTUs with $>99 \%$ 310 similarity to BOLD sequences revealed the presence of related sequences mainly in 311 Canada and France, but also in Moldova, Norway and South Africa (Table 1).

313 For Lepidocyrtus, five out of the 18 OTUs were singletons (Fig 3). Overall mean 314 divergence among OTUs was $14 \%$ (Table 2). Sequences assigned to the morphospecies 315 L. cyaneus and L. lanuginosus corresponded to nine and six OTUs respectively, while 316 those assigned to L. lignorum and L. curvicollis corresponded to three and two OTUs 317 respectively (Table 2, Fig. 3). Seven out of the 18 OTUs are comprised of individuals 318 representing more than one morphospecies, but are mainly represented by one (e.g. 
319 most individuals of OTU 3 are L. lignorum), apart from OTU 1 which is the most

320 abundant ( $\mathrm{n}=189$ specimens) and widespread ( $\mathrm{n}=41$ sites) OTU with very low sequence

321 variation (only 9 haplotypes), equally represented by two morphospecies, $L$.

322 lanuginosus and L. lignorum ( $\mathrm{n}=83$ and $\mathrm{n}=82$ respectively), plus a few specimens

323 assigned to L. curvicollis, L. cyaneus and L. ruber. The number of haplotypes per OTU

324 ranged from 1 to 14 (Table 2). The 13 OTUs with $>99 \%$ similarity to BOLD sequences

325 revealed the presence of related sequences mainly in Canada and France, but also in 326 Germany, Italy, Poland, USA, Australia and Tasmania (Table 2).

328 Geographical distributions

330 Entomobrya OTUs 1, 4, 11 and 12 are the most abundant (n individuals $>90$ ) and 331 widespread (n sampling sites $>30$ ) with ranges extending across the geographic extent 332 of sampling sites (Fig. 4 \& Appendix S3: Fig. S3.1). OTUs 5 and 6 have medium 333 abundances (n individuals $>50$ ) and are also relatively broadly distributed (Appendix 334 S3: Fig. S3.2). The remaining OTUs are represented by a very small number of 335 individuals and occur in a single or very few sampling sites (Appendix S3: Fig. S3.3). 336 Among the six abundant OTUs, only OTU 4 presented a pattern of non-random 337 geographic structuring of genetic variation (Fig. 2). Within this OTU, clear structuring 338 of genetic variation was found in two monophyletic clusters of DNA sequences, NivG1 339 and NivG2, comprised of ten individuals (7 haplotypes) and 6 individuals (3 340 haplotypes), respectively (posterior probability: NivG1 = 1; NivG2 =1) (Figs. $2 \& 4$ ). 341 Both clades are found in only a few geographically proximate sites in the North, 342 Northwest and Yorkshire regions. NIVG1 spans both glaciated and unglaciated areas, 
343 while NIVG2 occurs within glaciated areas only. The following disjunctive distributions 344 were found: North East, North West, Wales and East Anglia (OTU2), North East and 345 South West (OTU3), East Anglia and Wales (OTU10) (Appendix S3: Fig. S3.3).

347 Among Lepidocyrtus OTUs, three main spatial distribution patterns were identified: 348 OTUs either presented broad ranges ( $n=3$ OTUs), disjunct distributions $(n=3)$, or were 349 geographically restricted to a few closely located sites $(n=7)$ (Table 2$)$. The remaining 350 five OTUs were found in single sampling sites, frequently being represented by a single 351 individual (Fig. 3). The ranges of the widespread OTUs 1 and 15 extended across the 352 geographic extent of sampling sites, while OTU 9 was not sampled from more northerly 353 areas, being largely distributed from West Yorkshire down to eastern, western and 354 southern regions (Appendix S3: Fig. S3.4). The following disjunctive distributions were 355 found: North, Wales and South (OTU 11), East Anglia and South West (OTU 17), 356 North, Humberside, South, South East and South West (OTU 13) (Fig. 5a,b). Within 357 OTUs with geographically localised ranges, OTU 3 and 16 are only found in 358 unglaciated sites, while the remaining OTUs $(6,7,8,12$ and 18) are found in both 359 glaciated and unglaciated sites (Fig. 5c,d). Geographically widespread OTUs were also 360 the most abundant ( $\mathrm{n}>40$ individuals), while localised or geographically disjunct OTUs 361 presented mid to low abundances (Table 2). Unlike Entomobrya, no pattern of 362 geographic structuring of genetic variation was found within widespread OTUs. 363 However, geographical structuring of genetic variation was found in five out of the 364 seven geographically localised OTUs (OTUs 3, 7, 8, 12 and 18). These OTUs 365 (comprised of 7 to 17 individuals) presented some level of haplotype variation (from 366 four to eight haplotypes per OTU), which were found only in a few closely located sites 
367 (from two to five sites per OTU, Table 2). OTUs 6 and 16 were also geographically

368 restricted, but did not present sequence variation.

$370 \mathrm{~K}$ function plots for each geographically localised and disjunctly distributed OTU, for

371 both Entomobrya and Lepidocyrtus, revealed deviations from random distribution of 372 genetic variation, with empirical values with corrections greater than that expected for a 373 random distribution (Appendix S3: Figs. S3.5 \& S3.6).

374

375 OTU diversity analysis

376

377 For Entomobrya, a total of 43 sampling sites were located in areas covered by the ice 378 sheet, while 56 were located in areas that have not been covered by ice during 379 glaciations. The investigation of geographical variation in Entomobrya genetic diversity 380 revealed that overall OTU richness is equivalent in glaciated and unglaciated areas 381 (Table 3). After rarefying the unglaciated community data down to the abundance level 382 of the glaciated community, it was found that for 1000 random samples of 264 383 individuals, there was an average of 11 OTUs with a variance of 0.9 (Table 3 ). The 384 confidence interval indicates that $95 \%$ of the times that a random sample of 264 385 individuals is drawn from the unglaciated community, it is expected to find between 938612 different OTUs. The observed diversity of the glaciated community ( $n=9$ OTUs) is 387 inside this confidence interval, indicating these communities are equivalent in diversity. 388 Looking within OTUs, observed haplotype richness of the smaller communities fell 389 within the simulated 95\% confidence interval for all but one OTU (OTU 11 haplotype 390 richness was greater in glaciated than unglaciated areas) (Table 3). 
391

392 For Lepidocyrtus, a total of 34 sites were sampled within each of the glaciated and 393 unglaciated areas of the Great Britain (Fig. 1). Contrary to Entomobrya data, the 394 analysis revealed that Lepidocyrtus OTU richness is significantly different between 395 glaciated and unglaciated communities with greater richness found in southern areas 396 (Table 4). The observed diversity of the glaciated community ( $\mathrm{n}=11$ OTUs) is outside 397 the confidence interval (15-17 OTUs) and is below the simulated average, indicating 398 that OTU richness is smaller than expected in the glaciated areas. Looking within the 399 three widespread OTUs (1,9 and 15), observed haplotype richness of the less abundant 400 community fell within the simulated $95 \%$ confidence interval for all OTUs, indicating 401 that haplotype richness does not differ between glaciated and unglaciated communities 402 for these OTUs (Table 4).

403

404 MtDNA COI rate estimation and long-term persistence analysis 405

406 The best model of molecular evolution for sequences in the two Entomobrya 407 monophyletic groups detected within E. nivalis OTU 4 (NivG1 and NivG2) was T92. 408 Adopting the closest model in BEAST (TN93) and the estimated COI substitution rate 409 (0.0421 substitutions/site/myr), the age of the MRCA for NivG1 is 77,100 years ago $410(\mathrm{HPD}=21,700-142,300)$ and 42,700 years ago for NivG2 $(\mathrm{HPD}=38,350-94,700)$ 411 (Appendix S4).

412

413 The best model for Lepidocyrtus OTUs 3 and 8 was Kimura 2-parameter (K2), while for 414 OTUs 7, 12 and 18 it was T92. Adopting the closest models in BEAST (HKY- 
415 Hasegawa-Kishino-Yano for K2 and TN93 for T92), the mean age of the MRCA for

416 these OTUs ranged from 160,000 years ago $(\mathrm{HPD}=77,200-256,200)$ to 533,900 years

417 ago (HPD=355,600-720,700) (Table 5, Figs. S11 to S15). These deep divergence times

418 are more likely to be underestimates than overestimates (i.e. a fast mtDNA COI

419 substitution rate was used), and they substantially predate the LGM.

420

\section{DISCUSSION}

422

423 Glacial events greatly altered distributional ranges of European species as ice or

424 permafrost conditions pushed organisms southward or gradually restricted them to 425 various refuges (e.g. Taberlet et al., 1998; Hewitt, 2004). As a consequence, high levels 426 of endemic genetic variation are typically found in refugial areas (evidence of long-term 427 in situ evolution) (e.g. Provan \& Bennett, 2008; Tzedakis et al., 2013), and disjunct 428 modern distributions are observed in some taxa (e.g Stehlik et al., 2002; Willis \& van 429 Andel, 2004; Hedderson \& Nowell, 2006; Schmitt et al., 2006). These two basic 430 arguments - endemism and disjunction, have been used to support the glacial survival 431 hypothesis (e.g Brochmann et al., 2003) and they were investigated within the 432 Entomobrya and Lepidocyrtus genera in order to detect OTUs with signatures of long433 term persistence.

434

435 Within the broadly distributed Entomobrya OTU 4, a pattern of non-random geographic 436 structuring of genetic variation was observed (Fig. 2) in two monophyletic clusters of 437 DNA sequences. NivG1 and NivG2, comprised of ten individuals (7 haplotypes) and 6 438 individuals (3 haplotypes), respectively, presented clear structuring of genetic variation 
439 (Figs. 2 \& 4), occurring in only a few geographically proximate sites in the North, 440 Northwest and Yorkshire regions. Genetic signatures in Lepidocyrtus presented stronger 441 support for the glacial survival hypothesis. Five mtDNA COI Lepidocyrtus OTUs 442 contained geographically restricted sequence variation (Figs. $3 \& 5$ c,d) and these OTUs 443 represented 29 haplotypes distributed across 18 sampling sites (Fig. 5c,d). These sites 444 are mostly in unglaciated terrains, but include some glaciated areas (largely located near 445 the border of the ice sheet, except for OTU 7 for which sequence variation extends well 446 into the northern glaciated area).

448 Furthermore, three Entomobrya and three Lepidocyrtus OTUs presented disjunct 449 geographical distributions mainly between North-South and East-West regions (Fig. 450 5a,b \& Appendix S3: Fig. S3.3). While this disjunctive pattern could be explained by 451 long distance dispersal after ice sheet retreat, as noted for other taxa previously studied 452 in different contexts (e.g. Brochmann et al., 2003; Beatty \& Provan, 2013; Tzedakis et 453 al., 2013), it seems unlikely for Entomobrya and Lepidocyrtus (and Collembola in 454 general) due to their limited dispersal capability. This low dispersive ability has been 455 well demonstrated by the strong geographic structuring across very short distances 456 found for Lepidocyrtus lineages in previous molecular studies in the Mediterranean 457 Basin and Panama (Cicconardi et al., 2010, 2013), and other genera elsewhere (e.g. 458 Garrick et al., 2007, 2008; McGaughran et al., 2008). A more likely scenario is that 459 these represent native OTUs whose origin in Great Britain predates the last glaciation, 460 for which previously broader distributions were reduced by glacial onset, with 461 persistence in a limited number of ice-free areas in otherwise unfavourable habitat. 462 After glaciation, as the ice receded, deglaciated regions recolonised by a few leading 
463 front OTUs (Hewitt 1999) would effectively pre-empt space, reducing the opportunity

464 for other surviving OTUs to re-expand their populations. A similar scenario could also

465 explain the existence of endemic variation. Localised clades could have once been

466 widespread (i.e. same and related alleles found elsewhere), but went extinct in most of

467 their range in response to climate change. After ice retreat, they remained isolated or

468 colonized a few closely located sites.

469

470 Great Britain sequences that are genetically identical or nearly identical to sequences

471 sampled in remote continents are most plausibly explained as human introductions.

472 Passive dispersal of Collembola through human activity has been implicated in the

473 sharing of lineages across distant continents, e.g. Cerathophysella denticulata lineage

474 L3 found in Canada, South Africa, Australia and New Zealand (Porco et al., 2012), and

475 such introductions are considered to have mainly been accidental (e.g. in soil used as

476 ship ballast). Mitochondrial metagenomic data has also revealed island faunas to be

477 highly represented by introduced Collembola (Cicconardi et al. 2017). Our data set

478 presents similar examples, such as Entomobrya OTU 6, with sequences $100 \%$ similar

479 sampled in Canada, and South Africa and sequences $100 \%$ identical

480 to Lepidocyrtus OTU 9 sampled in Australia and Tasmania. Genetic identity, or near

481 identity across these vast geographic distances is consistent with passive transport

482 through human activities.

483

484 OTU diversity analysis

485 
486 Our results illustrate the extent to which reliance on morphospecies can misrepresent 487 diversity. Within Entomobrya, colour-pattern morphospecies were generally 488 monophyletic but concealing two or more mtDNA OTUs (Fig. 2). Katz et al. (2015) 489 also found colour pattern species of Entomobrya were generally (but not universally) 490 supported by mtDNA in the USA. The correspondence between morphospecies and 491 OTU was less clear for Lepidocyrtus. In particular, animals assigned to the 492 morphospecies Lepidocyrtus cyaneus featured in seven OTUs, and the dominant OTU 493 contained a mix of morphospecies (mainly L. lignorum and L. lanuginosus). 494 Unexpectedly high cryptic species diversity has been noted previously in this genus 495 (Ciccornadi et al., 2010, Cicconardi et al. 2013), and together these results suggest that 496 key taxonomic features (mainly distribution of scales, ground colour) may be unreliable 497 indicators of species boundaries.

499 If soil-dwelling mesofauna survived within southern regions that remained ice free, they 500 would be expected to exhibit higher species richness and higher genetic diversity within 501 species, compared to subsequently recolonised glaciated areas. Our analysis of genetic 502 diversity revealed different responses between the genera. Entomobrya presented 503 equivalent OTU richness between glaciated and unglaciated areas, providing no support 504 for the northern poverty hypothesis. In contrast, Lepidocyrtus OTU richness did differ 505 between glaciated and unglaciated communities, with fewer OTUs in the glaciated 506 areas, supporting the above hypothesis. Entomobrya and Lepidocyrtus may have 507 different rates of dispersal, which could potentially explain their distinct re-colonization 508 patterns. 
Long-term persistence of Entomobrya and Lepidocyrtus in the Great Britain

511

512 Our data reveal evidence for population persistence of Entomobrya OTUs within Great

513 Britain, estimated to extend back at least some 77,000 years. Six geographically

514 proximate sampling sites are represented exclusively by 9 haplotypes that comprise two

515 monophyletic groups, not sampled within any other site. These six sites (Fig. 4) span

516 both glaciated and unglaciated terrains of Great Britain, with most sequence variation

517 concentrated within the sampling sites extending well into the glaciated northern areas.

518 The estimated age of 77,000 years for the onset of genetic differentiation within the

519 oldest of these two clades indicates persistence through the last Pleistocene glaciation.

520

521 Similarly, with divergences dating back at least to the Ionian stage of the Pleistocene 522 ( $\sim 500-160$ Kya), Lepidocyrtus OTU differentiation is inferred to predate the LGM. The 523 implication is that the present distribution of the five geographically structured OTUs 524 has been influenced by relatively old paleogeographic events. These OTUs survived 525 through extreme climatic and ecological changes that characterized Great Britain and 526 have remained isolated from neighbouring OTUs.

527

528 The Collembola genera Lepidocyrtus and Entomobrya add to the growing evidence for 529 an ancient invertebrate fauna in Great Britain (Nieberding et al., 2005; McInerney et al., 530 2014, see above). Our results are consistent with other studies demonstrating the 531 persistence of Collembola lineages through extreme climatic disturbances, such as 532 glaciations in the Antarctic (Stevens et al., 2007), the Messinian Salinity Crisis in the 533 Mediterranean basin (Cicconardi et al., 2010) and periglacial forest contraction in 
534 southeastern Australia (Garrick et al., 2007). Together, these studies demonstrate the

535 marked resilience of the Collembola fauna to historical climate cycles, and their 536 suitability to investigate its consequences.

538 The unglaciated southern regions of the British Isles have been previously suggested to 539 act as northern cryptic refugia based upon evidence from fossil remains (Vincent, 1990), 540 fish (Bernatchez, 2001; Hänfling et al., 2002), and groundwater amphipods (McInerney 541 et al., 2014). Results presented here extend inference beyond the long-term persistence 542 for Collembola OTUs in unglaciated areas. Patterns of genetic variation within 543 Collembola also reveal evidence for population persistence within regions inferred to 544 have been glaciated, suggesting the existence of small ice-free northern areas that 545 allowed survival. One possible mechanism for the existence of northern ice-free areas in 546 Great Britain comes from recent work suggesting they may result from geothermal 547 activity, i.e. they are located close to geothermal heated ground and water (e.g. heated 548 ground and ponds, steam fields). These areas would have provided thermal gradients 549 with less extreme temperatures (between very high temperatures in the core of 550 geothermal sites, and very low temperatures below the ice-sheets), thus, possibly 551 creating a buffer zone where life could survive - the "geothermal glacial refugia" 552 hypothesis (Convey \& Lewis Smith, 2006). Such geothermal activity usually persists 553 over extended time scales that could encompass glacial cycles, providing refugia for life 554 (Fraser et al., 2014). Using Antarctica as a natural model system and a comprehensive 555 terrestrial database associated with landscape, climate and geothermal data, Fraser et al. 556 (2014) provided strong support for the role of geothermal heated terrain in structuring 557 broad-scale contemporary patterns in Antarctic diversity by providing glacial refugia. 
558 They further suggest that for many cases where the existence of small refugia within the

559 glaciated regions of northern Europe and northern North America has been inferred

560 from phylogeographic data, but their causes and precise locations have not been

561 identified, there is a great possibility that they may have been geothermal. A

562 consideration of the geothermal map of Great Britain (Busby et al., 2011) suggests that

563 some sampling sites where Entomobrya and Lepidocyrtus OTUs present signatures of in

564 situ diversification coincide with areas around geothermal terrains. For example, the

565 oldest E. nivalis OTUs (NivG1 and NivG2) were sampled from sampling sites 76, 77,

56678 and 79 , which broadly coincide with terrains that receive heat flow from the

567 Northern England Granites. Sites for L. cyaneus OTUs 7 (loc: 10, 101) and 12 (loc: 24,

568 28) coincide with areas near thermal springs (e.g. Taffs Well) and close to heat flow

569 from the Cheshire Basin. While these data suggest that long-term persistence of

570 Collembola in glaciated areas of the Great Britain may have been facilitated by

571 geothermal glacial refugia, this hypothesis remains to be tested.

572

573 ACKNOWLEDGEMENTS

574

575 This study was supported by SYNTAX grant awarded to P.S. C.M.A.F. was funded by a

576 Brazilian Council for Scientific and Technological Development PhD studentship 577 (CNPQ -200439/2010-3).

578

579 REFERENCES

580 Baddeley, A. \& Turner, R. (2005) spatstat: An R package for analyzing spatial point 581 patterns. Journal of Statistical Software, 12, 1-42. 
582 Beatty, G.E. \& Provan, J. (2013) Post-glacial dispersal, rather than in situ glacial

583

584

585

586

587

588

589

590

591

592

593

594

595

596

597

598

599

600

601

602

603

604

605

606

607

608

609

610

611

612

613

614

615

616

617

618

619

620 survival, best explains the disjunct distribution of the Lusitanian plant species Daboecia cantabrica (Ericaceae). Journal of Biogeography, 40, 335-344.

Bernatchez, L. (2001) The evolutionary history of brown trout (Salmo trutta L.) inferred from phylogeographic, nested clade, and mismatch analyses of mitochondrial DNA variation. Evolution, 55, 351-379.

Brochmann, C., Gabrielsen, T.M., Nordal, I., Landvik, J.Y., \& Elven, R. (2003) Glacial survival or tabula rasa? The history of North Atlantic biota revisited. Taxon, 52, 417-450.

Brower, A.V. (1994) Rapid morphological radiation and convergence among races of the butterfly Heliconius erato inferred from patterns of mitochondrial DNA evolution. Proceedings of the National Academy of Sciences, 91, 6491-6495.

Busby, J., Kingdon, A., \& Williams, J. (2011) The measured shallow temperature field in Britain. Quarterly Journal of Engineering Geology and Hydrogeology, 44, 373-387.

Chiverrell, R.C. \& Thomas, G.S.P. (2010) Extent and timing of the Last Glacial Maximum (LGM) in Britain and Ireland: a review. Journal of Quaternary Science, 25, 535-549.

Cicconardi, F., Borges, P.A.V., Strasberg, D., Oromí, P., López, H., Pérez-Delgado, A.J., Casquet, J., Caujapé-Castells, J., Fernández-Palacios, J.M., Thébaud, C., \& Emerson, B.C. (2017) MtDNA metagenomics reveals large-scale invasion of belowground arthropod communities by introduced species. Molecular Ecology, 26, 3104-3115.

Cicconardi, F., Fanciulli, P.P., \& Emerson, B.C. (2013) Collembola, the biological species concept and the underestimation of global species richness. Molecular Ecology, 22, 5382-5396.

Cicconardi, F., Nardi, F., Emerson, B.C., Frati, F., \& Fanciulli, P.P. (2010) Deep phylogeographic divisions and long-term persistence of forest invertebrates (Hexapoda: Collembola) in the North-Western Mediterranean basin. Molecular Ecology, 19, 386-400.

Clark, C.D., Hughes, A.L.C., Greenwood, S.L., Jordan, C., \& Sejrup, H.P. (2012) Pattern and timing of retreat of the last British-Irish Ice Sheet. Quaternary Science Reviews, 44, 112-146.

Convey, P. \& Lewis Smith, R. i. (2006) Geothermal bryophyte habitats in the South Sandwich Islands, maritime Antarctic. Journal of Vegetation Science, 17, 529538.

Coyer, J.A., Peters, A.F., Stam, W.T., \& Olsen, J.L. (2003) Post-ice age recolonization and differentiation of Fucus serratus L. (Phaeophyceae; Fucaceae) populations in Northern Europe. Molecular Ecology, 12, 1817-1829. 
DeSalle, R., Freedman, T., Prager, E.M., \& Wilson, A.C. (1987) Tempo and mode of sequence evolution in mitochondrial DNA of Hawaiian Drosophila. Journal of Molecular Evolution, 26, 157-164.

Dixon, P.M. (2002) Ripley's K function. Encyclopedia of environmetrics (ed. by A.H. El-Shaarawi and W.W. Piegorsch), Wiley, Chichester New York.

Drummond, A.J., Suchard, M.A., Xie, D., \& Rambaut, A. (2012) Bayesian Phylogenetics with BEAUti and the BEAST 1.7. Molecular Biology and Evolution, 29, 1969-1973.

Emerson, B.C., Cicconardi, F., Fanciulli, P.P., \& Shaw, P.J.A. (2011) Phylogeny, phylogeography, phylobetadiversity and the molecular analysis of biological communities. Philosophical Transactions of the Royal Society B: Biological Sciences, 366, 2391-2402.

Ezard, T., Fujisawa, T., \& Barraclough, T.G. (2009) SPLITS: SPecies' LImits by Threshold Statistics: R package version 1.0-18/r45.

Fraser, C.I., Terauds, A., Smellie, J., Convey, P., \& Chown, S.L. (2014) Geothermal activity helps life survive glacial cycles. Proceedings of the National Academy of Sciences of the United States of America, 111, 5634-5639.

Fujisawa, T. \& Barraclough, T.G. (2013) Delimiting species using single-locus data and the Generalized Mixed Yule Coalescent approach: a revised method and evaluation on simulated data sets. Systematic Biology, 62, 707-724.

Garrick, R.C., Rowell, D.M., Simmons, C.S., Hillis, D.M., \& Sunnucks, P. (2008) Finescale phylogeographic congruence despite demographic incongruence in two low-mobility saproxylic springtails. Evolution, 62, 1103-1118.

Garrick, R.C., Sands, C.J., Rowell, D.M., Hillis, D.M., \& Sunnucks, P. (2007) Catchments catch all: long-term population history of a giant springtail from the southeast Australian highlands - a multigene approach. Molecular Ecology, 16, $1865-1882$.

Gibert, J., Danielopol, D., \& Stanford, J.A. (1994) Groundwater Ecology. Academic Press, San Diego, California.

Gotelli, N.J. \& Entsminger, G.L. (2000) EcoSim: Null models software for ecology. Version 5.0. Acquired Intelligence Inc. \& Kesey-Bear,

Hänfling, B., Hellemans, B., Volckaert, F. a. M., \& Carvalho, G.R. (2002) Late glacial history of the cold-adapted freshwater fish Cottus gobio, revealed by microsatellites. Molecular Ecology, 11, 1717-1729.

Hedderson, T.A. \& Nowell, T.L. (2006) Phylogeography of Homalothecium sericeum (Hedw.) Br. Eur.; toward a reconstruction of glacial survival and postglacial migration. Journal of Bryology, 28, 283-292. 
658 Hewitt, G. (2000) The genetic legacy of the Quaternary ice ages. Nature, 405, 907-913.

659 Hewitt, G.M. (1999) Post-glacial re-colonization of European biota. Biological Journal 660 of the Linnean Society, 68, 87-112.

661 Hewitt, G.M. (2004) Genetic consequences of climatic oscillations in the Quaternary. 662 Philosophical Transactions of the Royal Society of London. Series B: Biological $663 \quad$ Sciences, 359, 183-195.

664 Ho, S.Y.W. \& Lo, N. (2013) The insect molecular clock. Australian Journal of 665 Entomology, 52, 101-105.

666 Hoarau, G., Coyer, J.A., Veldsink, J.H., Stam, W.T., \& Olsen, J.L. (2007) Glacial 667 refugia and recolonization pathways in the brown seaweed Fucus serratus. 668 Molecular Ecology, 16, 3606-3616.

669 Hopkin, S.P. (1997) Biology of the Springtails: (Insecta: Collembola). Oxford 670

Hopkin, S.P. (2007) A Key to the Collembola (springtails) of Britain and Ireland. Field

673

674

675

676

677 678 679

680 681 682 683

684 685 686 687 688

689

690 691

692

693 694

Kapli, P., Lutteropp, S., Zhang, J., Kobert, K., Pavlidis, P., Stamatakis, A., \& Flouri, T. (2017) Multi-rate Poisson Tree Processes for single-locus species delimitation under Maximum Likelihood and Markov Chain Monte Carlo. Bioinformatics, 33, 1630-1638.

Katoh, K., Misawa, K., Kuma, K., \& Miyata, T. (2002) MAFFT: a novel method for rapid multiple sequence alignment based on fast Fourier transform. Nucleic Acids Research, 30, 3059-3066.

Katz, A.D., Giordano, R., \& Soto-Adames, F.N. (2015) Operational criteria for cryptic species delimitation when evidence is limited, as exemplified by North American Entomobrya (Collembola: Entomobryidae). Zoological Journal of the Linnean Society, 173, 818-840.

Kearse, M., Moir, R., Wilson, A., Stones-Havas, S., Cheung, M., Sturrock, S., Buxton, S., Cooper, A., Markowitz, S., Duran, C., Thierer, T., Ashton, B., Meintjes, P., \& Drummond, A. (2012) Geneious Basic: An integrated and extendable desktop software platform for the organization and analysis of sequence data. Bioinformatics, 28, 1647-1649.

Luo, A., Ling, C., Ho, S.Y.W., Zhu, C.-D., \& Mueller, R. (2018) Comparison of methods for nolecular species delimitation across a range of speciation scenarios. Systematic Biology, 67, 830-846.

McGaughran, A., Hogg, I.D., \& Stevens, M.I. (2008) Patterns of population genetic structure for springtails and mites in southern Victoria Land, Antarctica. Molecular Phylogenetics and Evolution, 46, 606-618. 
706

707

708

709

710

711

712

713

714

715

716

717

718

719

720

721

722

723

724

725

726

727

728

729

730

731

McInerney, C.E., Maurice, L., Robertson, A.L., Knight, L.R.F.D., Arnscheidt, J., Venditti, C., Dooley, J.S.G., Mathers, T., Matthijs, S., Eriksson, K., Proudlove, G.S., \& Hänfling, B. (2014) The ancient Britons: groundwater fauna survived extreme climate change over tens of millions of years across NW Europe. Molecular Ecology, 23, 1153-1166.

Montgomery, W.I., Provan, J., McCabe, A.M., \& Yalden, D.W. (2014) Origin of British and Irish mammals: disparate post-glacial colonisation and species introductions. Quaternary Science Reviews, 98, 144-165.

Nieberding, C., Libois, R., Douady, C.J., Morand, S., \& Michaux, J.R. (2005) Phylogeography of a nematode (Heligmosomoides polygyrus) in the western Palearctic region: persistence of northern cryptic populations during ice ages? Molecular Ecology, 14, 765-779.

Porco, D., Bedos, A., Greenslade, P., Janion, C., Skarżyński, D., Stevens, M.I., Jansen van Vuuren, B., \& Deharveng, L. (2012) Challenging species delimitation in Collembola: cryptic diversity among common springtails unveiled by DNA barcoding. Invertebrate Systematics, 26, 470-477.

Provan, J. \& Bennett, K.D. (2008) Phylogeographic insights into cryptic glacial refugia. Trends in Ecology \& Evolution, 23, 564-571.

Provan, J., Wattier, R.A., \& Maggs, C.A. (2005) Phylogeographic analysis of the red seaweed Palmaria palmata reveals a Pleistocene marine glacial refugium in the English Channel. Molecular Ecology, 14, 793-803.

QGIS Development Team (2014) QGIS Geographic Information System. Open Source Geospatial Foundation Project,

Rambaut, A., Suchard, M.A., Xie, D., \& Drummond, A.J. (2014) Tracer v1.6.

Ramirez-Gonzalez, R., Yu, D.W., Bruce, C., Heavens, D., Caccamo, M., \& Emerson, B.C. (2013) PyroClean: denoising pyrosequences from protein-coding amplicons for the recovery of interspecific and intraspecific genetic variation. PLOS ONE, 8, 1-11.

Schmitt, T., Hewitt, G.M., \& Müller, P. (2006) Disjunct distributions during glacial and interglacial periods in mountain butterflies: Erebia epiphron as an example. Journal of Evolutionary Biology, 19, 108-113.

Searle, J.B. (2008) The colonization of Ireland by mammals. The Irish Naturalists' Journal, 29, 109-115.

South, A. (1961) The Taxonomy of the British Species of Entomobrya (collembola). Transactions of the Royal Entomological Society of London, 113, 387-416.

Stamatakis, A., Hoover, P., \& Rougemont, J. (2008) A rapid bootstrap algorithm for the RAxML Web servers. Systematic Biology, 57, 758-771. 
732

733

734

735

736

737

738

739

740

741

742

743

744

745

746

747

748

749

750

751

752

753

754

755

756

757

758

759

760

761

762

763

764

765

766

Stehlik, I., Blattner, F.R., Holderegger, R., \& Bachmann, K. (2002) Nunatak survival of the high Alpine plant Eritrichium nanum (L.) Gaudin in the central Alps during the ice ages. Molecular Ecology, 11, 2027-2036.

Stevens, M.I., Frati, F., McGaughran, A., Spinsanti, G., \& Hogg, I.D. (2007) Phylogeographic structure suggests multiple glacial refugia in northern Victoria Land for the endemic Antarctic springtail Desoria klovstadi (Collembola, Isotomidae). Zoologica Scripta, 36, 201-212.

Taberlet, P., Fumagalli, L., Wust-Saucy, A.-G., \& Cosson, J.-F. (1998) Comparative phylogeography and postglacial colonization routes in Europe. Molecular Ecology, 7, 453-464.

Tamura, K., Stecher, G., Peterson, D., Filipski, A., \& Kumar, S. (2013) MEGA6: Molecular Evolutionary Genetics Analysis Version 6.0. Molecular Biology and Evolution, 30, 2725-2729.

Teacher, A.G.F., Garner, T.W.J., \& Nichols, R.A. (2009) European phylogeography of the common frog (Rana temporaria): routes of postglacial colonization into the British Isles, and evidence for an Irish glacial refugium. Heredity, 102, 490-496.

Tzedakis, P.C., Emerson, B.C., \& Hewitt, G.M. (2013) Cryptic or mystic? Glacial tree refugia in northern Europe. Trends in Ecology \& Evolution, 28, 696-704.

Villesen, P. (2007) FaBox: an online toolbox for fasta sequences. Molecular Ecology Notes, 7, 965-968.

Vincent, P. (1990) The Biogeography of the British Isles: An Introduction. Routledge, London; New York.

Wares, J.P. (2001) Patterns of Speciation Inferred from Mitochondrial DNA in North American Chthamalus (Cirripedia: Balanomorpha: Chthamaloidea). Molecular Phylogenetics and Evolution, 18, 104-116.

Willis, K.J. \& van Andel, T.H. (2004) Trees or no trees? The environments of central and eastern Europe during the Last Glaciation. Quaternary Science Reviews, 23, $2369-2387$.

Yalden, D.W. (1982) When did the mammal fauna of the British Isles arrive? Mammal Review, 12, 1-57.

\section{DATA ACCESSIBILITY}

DNA sequences were deposited in BoldSystem (http://www.boldsystems.org/) under accession numbers XXX 


\section{BIOSKETCH}

768 Christiana M. A. Faria is currently a postdoctoral researcher at the laboratory of

769 Ecology and Evolution of Social Insects at the University of Ceará, Brazil. She is

770 interested in the application of molecular methods to the study of ecology, behaviour,

771 biogeography, species adaptation and diversification processes.

772

\section{AUTHOR CONTRIBUTIONS}

774 C.M.A.F and B.C.E. designed the research, P.S. collected the samples, C.M.A.F.

775 conducted the laboratory work and analysed the data. C.M.A.F and B.C.E wrote the 776 paper, with input from P.S.

777

778 SUPPORTING INFORMATION

779 Appendix S1 Supplementary information on the materials used in this study

780 Appendix S2 Supplementary information for methods and results used in this study

781 Appendix S3 Spatial distribution patterns for Entomobrya and Lepidocyrtus OTUs

782 Appendix S4 Dated trees for Entomobrya and Lepidocyrtus geographically localized

783 monophyletic clusters

784

785 
786 Table 1. Summary data for each Entomobrya OTU sampled in Great Britain, reporting the number of individuals (n ind) and of mtDNA COI 787 haplotypes (n hap) found, the number of sites each OTU was sampled from (n sites), the number of sequences sampled from single localities ( $\mathrm{n}$ 788 single), the number of localities sampled in glaciated ( $\mathrm{n}$ glac) and unglaciated ( $\mathrm{n}$ unglac) areas, the mean and maximum observed intraspecific $\mathrm{p}$ 789 distances, and BOLD information on the locality of occurrence of the identical or near identical sequence found in this database ( $>99 \%$ 790 similarity). 'No match' a query sequence has no hit in Bold database. 791

\begin{tabular}{|c|c|c|c|c|c|c|c|c|c|c|}
\hline OTU & morphospecies & $\mathrm{n}$ ind & $\mathrm{n}$ hap & $\mathrm{n}$ sites & $\mathrm{n}$ single & $\mathrm{n}$ glac & $\mathrm{n}$ unglac & Range & $\begin{array}{c}\text { Mean/max } \\
\text { intra-OTU p-dist }\end{array}$ & $\begin{array}{l}\text { BOLD most frequent } \\
\text { sequence }\end{array}$ \\
\hline 1 & intermedia $^{a}$ & 165 & 22 & 47 & 11 & 21 & 26 & widespread & $2.1 / 4$ & Canada \\
\hline 2 & intermedia & 5 & 2 & 5 & 1 & 3 & 2 & disjunct & $0.1 / 0.2$ & Canada \\
\hline 3 & nivalis/marginata & 2 & 2 & 2 & 2 & 1 & 1 & disjunct & $0.2 / 0.2$ & no match \\
\hline 4 & nivalis $^{b}$ & 94 & 38 & 33 & 29 & 12 & 21 & widespread & $1.3 / 3.2$ & France, Canada \\
\hline 5 & nivalis $^{c}$ & 54 & 16 & 24 & 12 & 6 & 18 & spread & $0.4 / 1.6$ & Norway \\
\hline 6 & mutifasciata $^{d}$ & 52 & 7 & 14 & 4 & 8 & 6 & spread & $0.1 / 0.5$ & France, Canada, SAfrica \\
\hline 7 & nivalis & 1 & 1 & 1 & 1 & 0 & 1 & - & - & no match \\
\hline
\end{tabular}




$\begin{array}{llccccccccc}8 & \text { nicoleti } & 2 & 2 & 1 & 0 & 1 & 0 & - & 1.4 / 1.4 & \text { no match } \\ 9 & \text { marginata } & 3 & 2 & 2 & 2 & 0 & 2 & - & 2.4 / 3.6 & \text { no match } \\ 10 & \begin{array}{l}\text { multifasciata } \\ \text { nicoleti }\end{array} & 2 & 2 & 2 & 2 & 0 & 21 & \text { disjunct } & - & \text { France, Canada } \\ 11 & 106 & 44 & 33 & 37 & 16 & 17 & \text { widespread } & 0.3 / 1.6 & \text { no match } \\ 12 & \text { albocinctaf } & 172 & 37 & 55 & 27 & 15 & 40 & \text { widespread } & 1.2 / 2.9 & \text { France, Canada, Moldova }\end{array}$

OTUs are mostly composed of one morphospecies but individuals from different morphospecies are also found within these OTUs:

aintermedia $(\mathrm{n}=160)$, nivalis $(\mathrm{n}=3)$, albocincta $(\mathrm{n}=1)$, multifasciata $(\mathrm{n}=1),{ }^{\mathrm{b}}$ ivivalis $(\mathrm{n}=86)$, intermedia $(\mathrm{n}=2)$, multifasciata $(\mathrm{n}=6),{ }^{\circ}$ nivalis $(\mathrm{n}=49)$, intermedia $(\mathrm{n}=4)$, marginata $(\mathrm{n}=1),{ }_{\text {multifasciata }}(\mathrm{n}=50)$, intermedia $(\mathrm{n}=1)$, nicoleti $(\mathrm{n}=1),{ }_{\text {enicoleti }}(\mathrm{n}=92)$, intermedia $(\mathrm{n}=4)$, multifasciata $(\mathrm{n}=7)$, nivalis $(\mathrm{n}=3)$, falbocincta $(\mathrm{n}=171)$, intermedia $(\mathrm{n}=1)$ 
804 Table 2. Summary data for each Lepidocyrtus OTU sampled in Great Britain, reporting: the number of individuals (n ind), number of mtDNA 805 COI haplotypes (n hap) found, number of sites each OTU was sampled from (n sites), number of localities sampled in glaciated (n glac) and 806 unglaciated (n unglac) areas, the mean and maximum observed intraspecific p-distances, and BOLD information on the locality of occurrence of 807 the identical or near identical sequence found in this database ( $>99 \%$ similarity). 'No match' a query sequence has no hit in Bold database, 'not 808 provided' the geographic information of similar sequence found in the database is missing, ' $<99 \%$ match' a similar sequence was found in the 809 database but it is less than $99 \%$ identical thus inhibiting taxonomic or geographic assignments.

\begin{tabular}{|c|c|c|c|c|c|c|c|c|c|}
\hline OTU & Morphospecies & $\mathrm{n}$ ind & $\mathrm{n}$ hap & $\mathrm{n}$ sites & n glac & n unglac & Range & $\begin{array}{l}\text { Mean/max } \\
\text { intra-OTU } \\
\text { p-distances }\end{array}$ & $\begin{array}{l}\text { BOLD most frequent } \\
\text { sequence }\end{array}$ \\
\hline 1 & lanuginosus/lignorum $^{a}$ & 189 & 9 & 41 & 25 & 16 & widespread & $0.02 / 0.5$ & Canada \\
\hline 2 & lignorum & 2 & 1 & 1 & 0 & 1 & & - & France \\
\hline 3 & lignorum/curvicollis $^{\mathrm{b}}$ & 9 & 7 & 4 & 0 & 4 & & $0.86 / 1.65$ & not provided \\
\hline 4 & albocincta & 1 & 1 & 1 & 0 & 1 & - & - & $<99 \%$ match \\
\hline 5 & lanuginosus & 1 & 1 & 1 & 1 & 0 & - & - & $<99 \%$ match \\
\hline 6 & lanuginosus & 13 & 2 & 4 & 3 & 1 & localised & $0.07 / 0.18$ & France \\
\hline 7 & cyaneus & 17 & 8 & 5 & 4 & 1 & - & $1.76 / 4.14$ & Canada \\
\hline
\end{tabular}




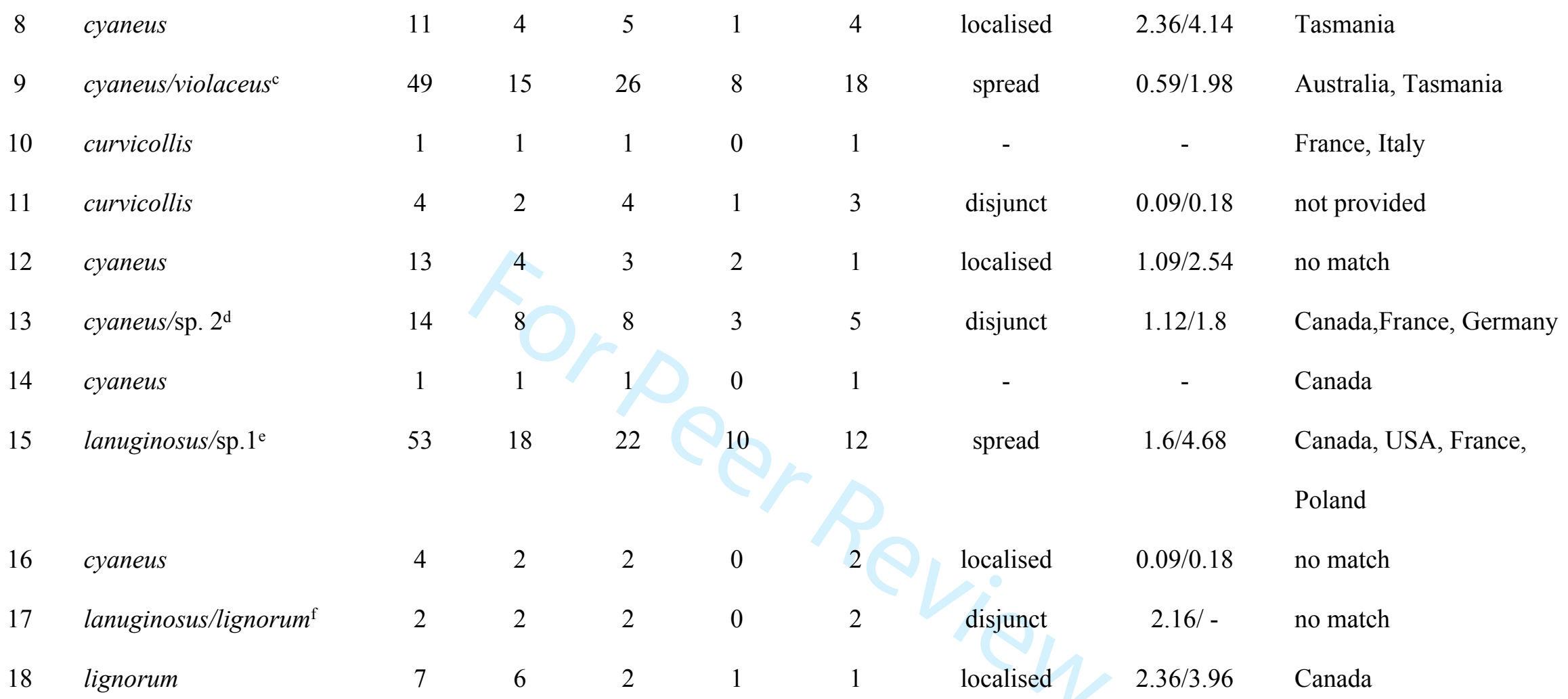

811 OTUs composed of individuals from more than one morphospecies: a lanuginosus $(\mathrm{n}=83)$, lignorum $(\mathrm{n}=82)$, curvicollis $(\mathrm{n}=13)$, cyaneus $(\mathrm{n}=9)$, ruber $(\mathrm{n}=1)$; 812 lignorum $(\mathrm{n}=5)$, curvicollis $(\mathrm{n}=3)$, lanuginosus $(\mathrm{n}=1) ;{ }^{\mathrm{c}}$ cyaneus $(\mathrm{n}=35)$, violaceus $(\mathrm{n}=1) ;{ }^{\mathrm{d}}$ cyaneus $(\mathrm{n}=13)$, Lepidocyrtus $\mathrm{sp} .2$ ( $\left.\mathrm{n}=1\right) ;{ }^{\mathrm{e}}$ lanuginosus $(\mathrm{n}=33)$,

813 Lepidocyrtus $\mathrm{sp} .1(\mathrm{n}=9)$, lignorum $(\mathrm{n}=5)$, cyaneus $(\mathrm{n}=2)$, curvicollis $(\mathrm{n}=1) ;{ }^{\mathrm{f}}$ lanuginosus $(\mathrm{n}=1)$, lignorum $(\mathrm{n}=1)$. 
815 Table 3. Summary of Entomobrya ECOSIM data entry and diversity results (mean and 95\% confidence interval - CI) for the overall OTU richness 816 and for each of the most abundant OTUs. For all simulated pairs, the larger community was rarefied down to the abundance level of the smaller 817 community being compared and the observed diversity (n OTU/hap) of the smaller community was tested to see if it fell within the estimated $81895 \%$ confidence interval of the larger community. $\mathrm{N}$ ind = number of individuals, $\mathrm{N}$ otu/hap = observed number of OTUs or of haplotypes within 819 OTUs, glac $=$ community in the glaciated area, unglac $=$ community in the unglaciated area. *Community is used in a statistical sense (not 820 ecological sense) meaning the collection of Entomobrya OTUs or collection of haplotypes within OTUs found in each area within Great Britain. 821

\begin{tabular}{|c|c|c|c|c|c|c|c|c|c|c|c|c|c|c|}
\hline & \multicolumn{2}{|c|}{ All OTUs } & \multicolumn{2}{|c|}{ OTU 1} & \multicolumn{2}{|c|}{ OTU 4} & \multicolumn{2}{|c|}{ OTU 5} & \multicolumn{2}{|c|}{ OTU 6} & \multicolumn{2}{|c|}{ OTU 11} & \multicolumn{2}{|c|}{ OTU 12} \\
\hline & glac & unglac & glac & unglac & glac & unglac & glac & unglac & glac & unglac & glac & unglac & glac & unglac \\
\hline $\mathrm{N}$ ind & 264 & 394 & 75 & 87 & 30 & 63 & 14 & 40 & 20 & 32 & 58 & 50 & 31 & 141 \\
\hline $\mathrm{N}$ otu/hap & 9 & 12 & 14 & 16 & 15 & 26 & 6 & 12 & 3 & 6 & 27 & 20 & 6 & 33 \\
\hline Mean & - & 11 & - & 14.7 & - & 16 & - & 6.3 & - & 4.4 & 23.7 & - & - & 10 \\
\hline $95 \%$ CI & - & 9 to 12 & - & 13 to 16 & - & 13 to 20 & - & 4 to 9 & - & 2 to 6 & 21 to 26 & - & - & 6 to 14 \\
\hline
\end{tabular}


824 Table 4. Summary of Lepidocyrtus ECOSIM data entry and diversity results (mean and $82595 \%$ confidence interval - CI) for overall OTU richness and for each of the most 826 abundant OTUs. For all simulated pairs, the larger community was rarefied down to the 827 abundance level of the smaller community being compared and the observed diversity 828 ( $n$ hap) of the smaller community was tested to see if it felt within the estimated 95\% 829 confidence interval of the larger community. $\mathrm{n}$ ind $=$ number of individuals, $\mathrm{n}$ otu/hap $=$ 830 number of OTUs or of haplotypes within OTUs, glac = community in the glaciated area, 831 unglac $=$ community in the unglaciated area. * Community is used in a statistical sense 832 (not ecological sense) meaning the collection of Lepidocyrtus OTUs or collection of 833 haplotypes within OTUs found in each area within Great Britain.

\begin{tabular}{lcccccccc}
\hline & \multicolumn{2}{c}{ All OTUs } & \multicolumn{2}{c}{ OTU 1 } & \multicolumn{2}{c}{ OTU 9 } & \multicolumn{2}{c}{ OTU 15 } \\
& glac & unglac & glac & unglac & glac & unglac & glac & unglac \\
\hline n ind & 184 & 205 & 117 & 72 & 15 & 34 & 19 & 34 \\
n otu/hap & 11 & 17 & 8 & 3 & 6 & 13 & 9 & 13 \\
Mean & - & 16.5 & 6 & - & - & 7 & - & 9 \\
$95 \%$ CI & - & 15 to 17 & 3 to 8 & - & - & 4 to 9 & - & 7 to 11
\end{tabular}

834

835

836

837

838

839

840

841 
842 Table 5. Estimated times, in thousands of years, of the MRCA of geographically 843 localized Lepidocyrtus mitochondrial OTUs (in situ endemic variation) sampled across 844 Great Britain expressed as mean values with 95\% highest posterior density (HPD) 845 intervals.

846

\begin{tabular}{lll}
\hline OTU & Mean value & $\mathbf{9 5 \% ~ H P D ~}$ \\
\hline OTU 3 & 160,000 & $77,200-256,200$ \\
OTU 7 & 533,390 & $355,600-720,700$ \\
OTU 8 & 424,200 & $257,720-610,100$ \\
OTU 12 & 236,800 & $129,400-359,600$ \\
OTU 18 & 451,400 & $280,000-655,100$
\end{tabular}

847

848

849

850

851

852

853

854

855

856

857

858

859 
FIGURE LEGENDS

861

862 Figure 1. Distribution of sampling sites in Great Britain; a complete list of geographic

863 coordinates and number of individuals sampled per locality can be found in Appendix

864 S1. Black triangles indicate the maximum extent of the British-Irish ice sheet during the

865 last Pleistocene glacial period, according to Clark et al. (2012)

866

867 Figure 2. Bayesian tree of Entomobrya mtCOI Sanger sequences $(\mathrm{n}=174$ unique

868 haplotypes) derived from 90 sampling sites across Great Britain (see Fig. 1, Appendix

869 S1). Katianna sp. was sampled as an outgroup. Numbers immediately to the right of 870 morphospecies names correspond to sampling sites. Two monophyletic clusters of DNA 871 sequences, NivG1 and NivG2, are grey highlighted which represents a clear structuring 872 of genetic variation found within OTU 4. Clades were collapsed to reduce space and the 873 sizes of the triangles indicate the amount of sequence variation within the clade. Further 874 OTU details can be found in Table 1.

875

876 Figure 3. Bayesian tree of Lepidocyrtus mtCOI Sanger sequences $(\mathrm{n}=98$ unique 877 haplotypes) derived from 68 sampling sites across Great Britain (see Fig. 1, Appendix 878 S1). Katianna sp. was sampled as an outgroup. Numbers immediately to the right of 879 morphospecies names correspond to sampling sites. Geographically localized OTUs are 880 grey highlighted, except OTUs 6 and 16 because they did not present sequence 881 variation. Most abundant OTUs are collapsed to reduce space and the sizes of the 882 triangles indicate the amount of sequence variation within the clade. Further OTU 883 details can be found in Table 2. 
884

885 Figure 4. Geographical distributions of Entomobrya a) OTU 1 - grey squares and b) 886 OTU 4 - grey dots, according to their sampling sites in Great Britain. Ellipses in b) 887 indicate geographically localized ranges of two monophyletic groups (NIVG1 - big 888 ellipse, and NIVG2 - small ellipse restricted to the glaciated area) found in OTU 4 (see 889 text for details). Black small dots indicate remaining sampling sites. Black triangles 890 indicate the maximum extent of the British-Irish ice sheet during the last Pleistocene 891 glacial period, according to Clark et al. (2012).

892

893 Figure 5. Geographical distributions of Lepidocyrtus OTUs with disjunctive 894 distributions in Great Britain: a) OTU 11 - black circles, OTU 17 = black squares, b) 895 OTU 13 - black circles and Lepidocyrtus OTUs with geographically localized 896 distributions c) OTU 3 - black squares, OTU 6 = black circles, OTU 7 = white stars, 897 OTU $18=$ black triangles, d) OTU $8=$ grey circles, OTU $12=$ black squares, OTU $16=$ 898 white stars. Overlapping shapes share same sampling sites. Small light grey dots 899 indicate remaining sampling sites. Light grey triangles indicate the maximum extent of 900 the British-Irish ice sheet during the last Pleistocene glacial period, according to Clark 901 et al. (2012). 


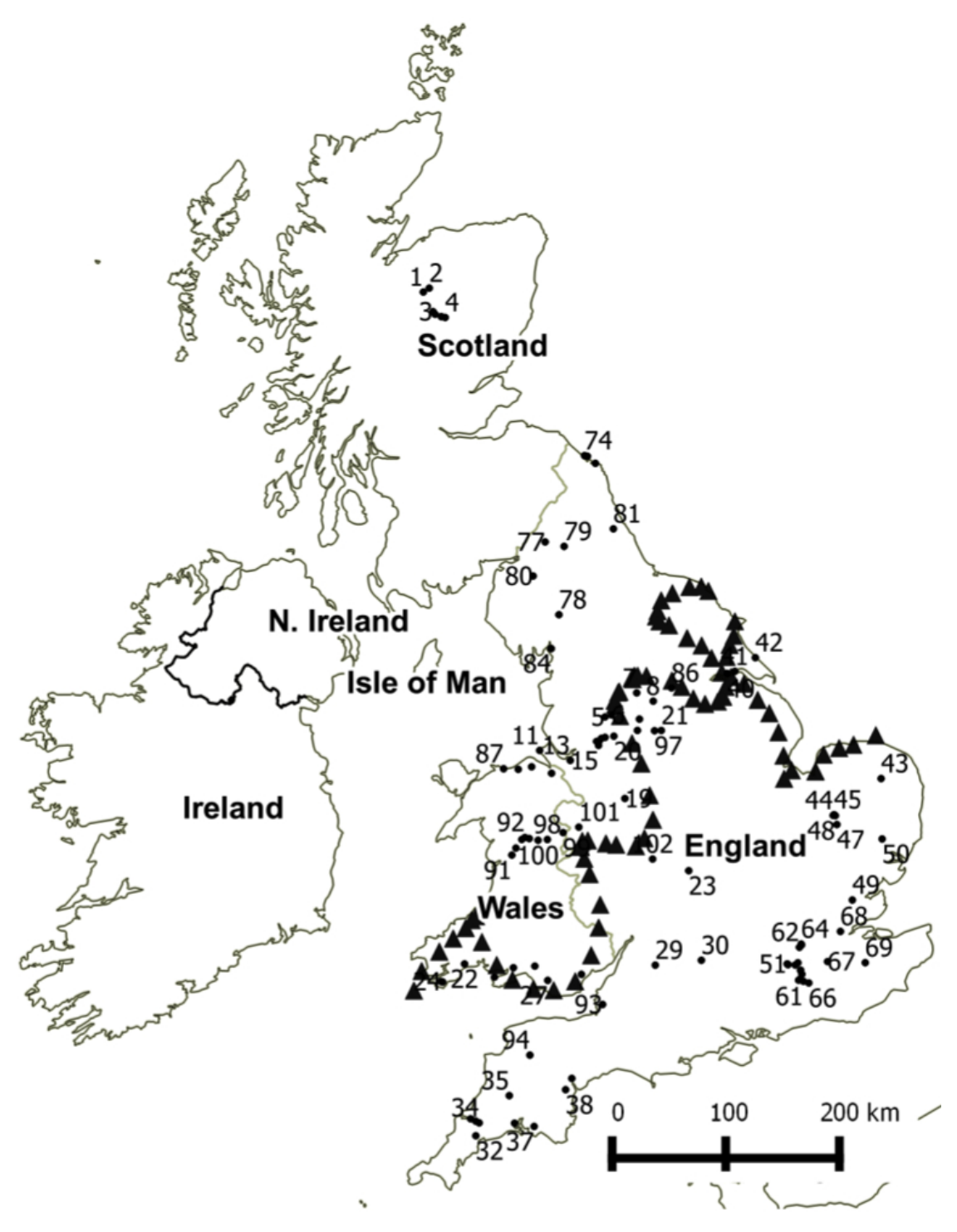

Figure 1 


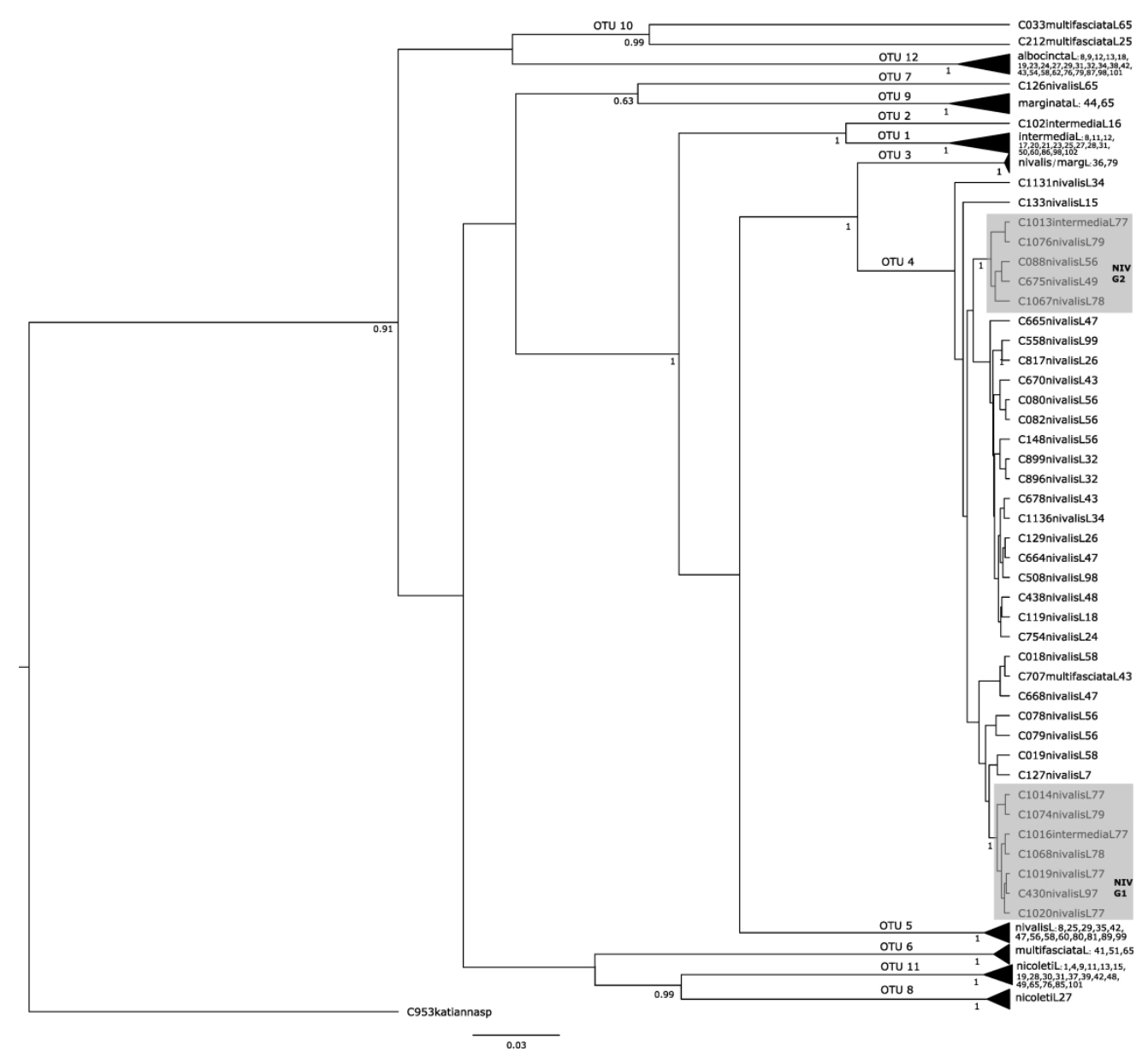

Figure 2 


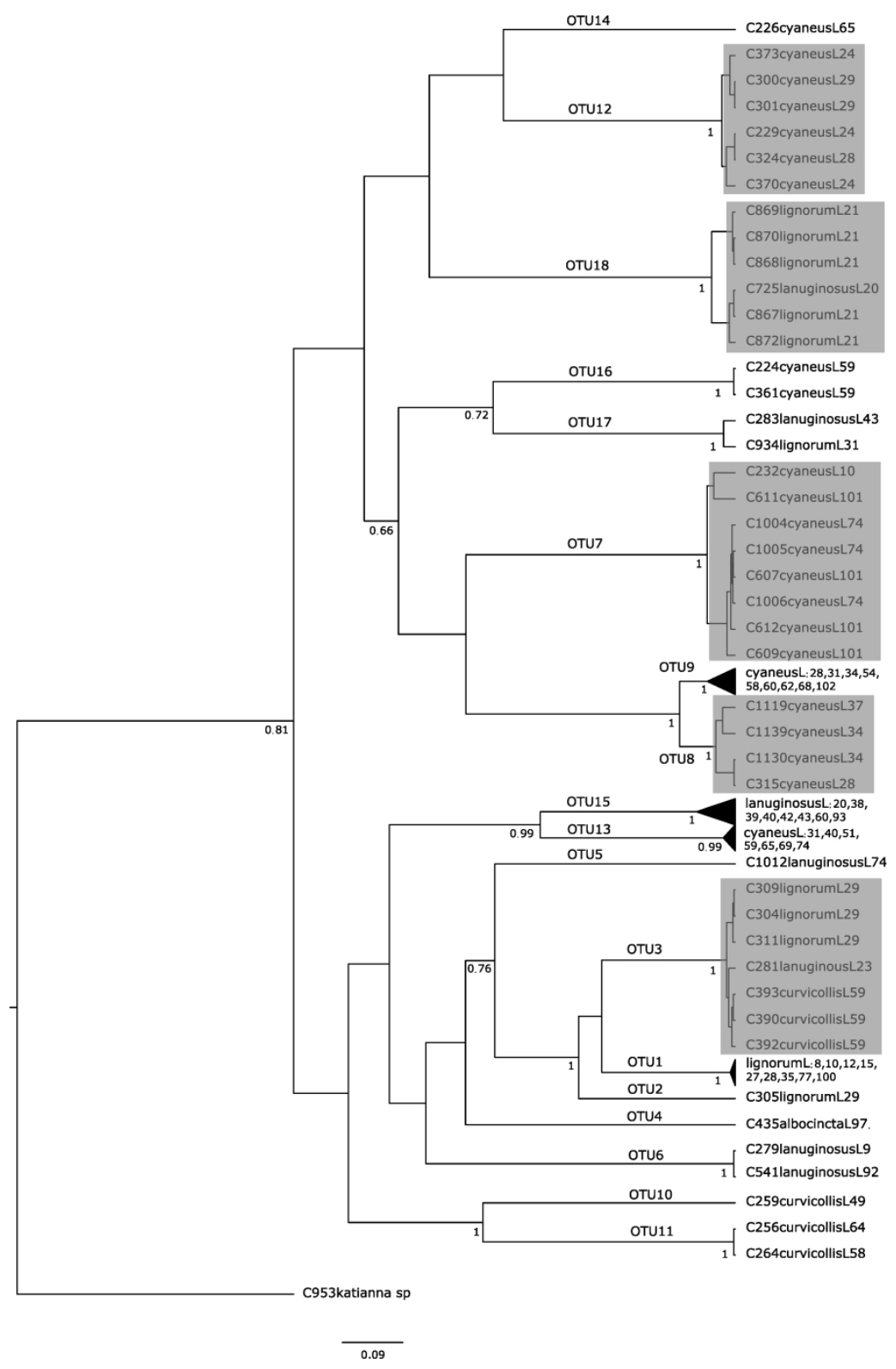

Figure 3 
(a)

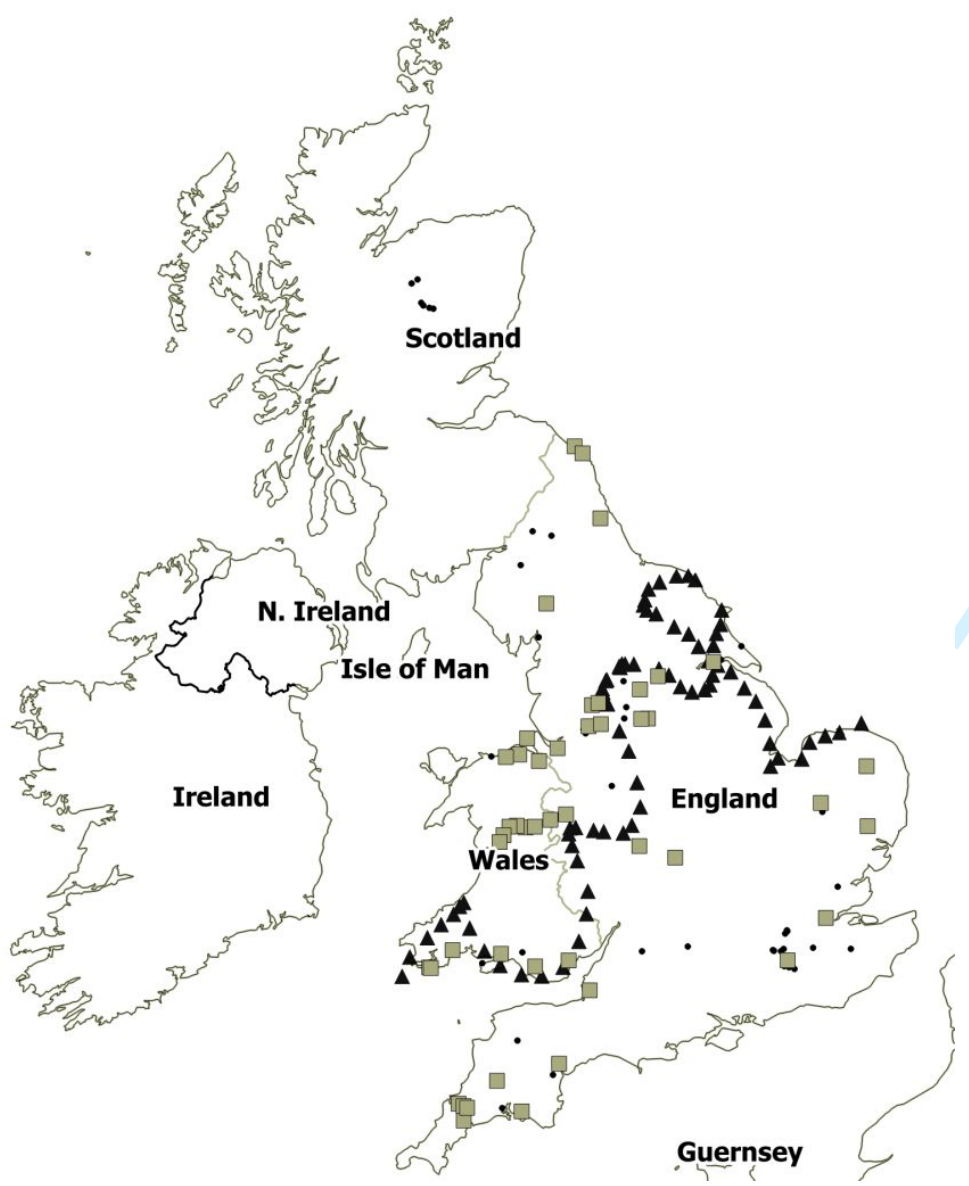

(b)

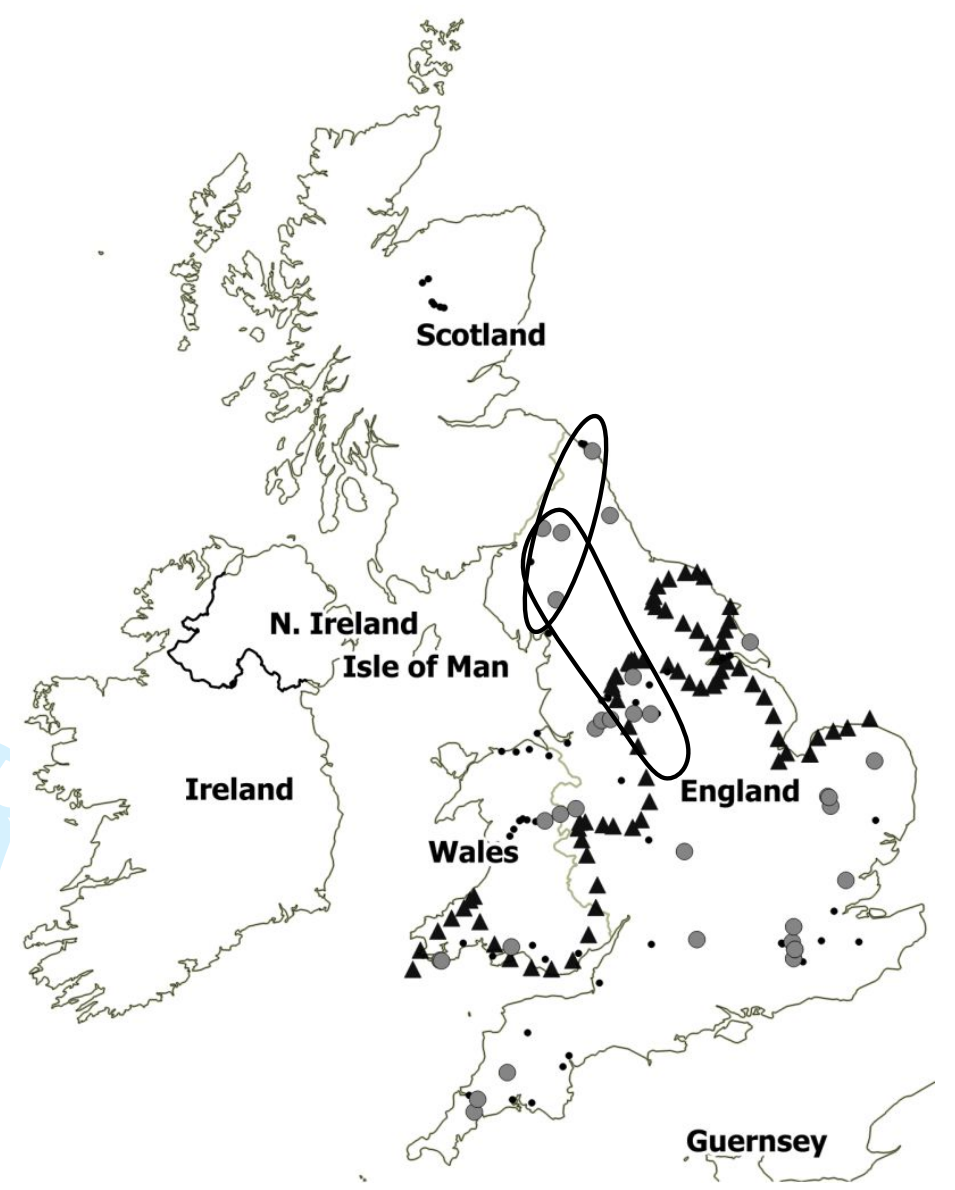

Figure 4 


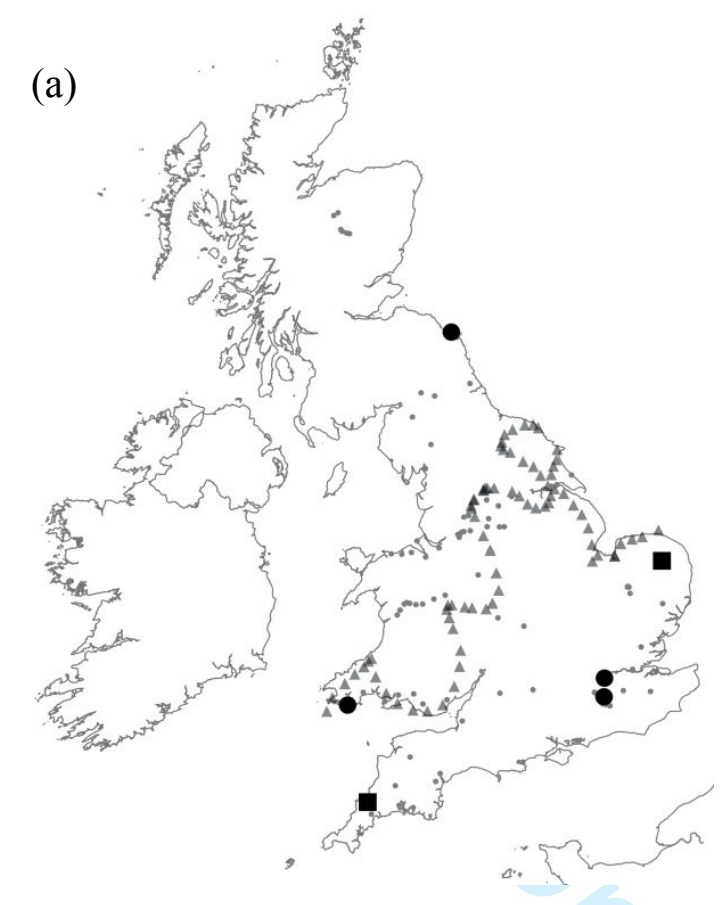

(b)
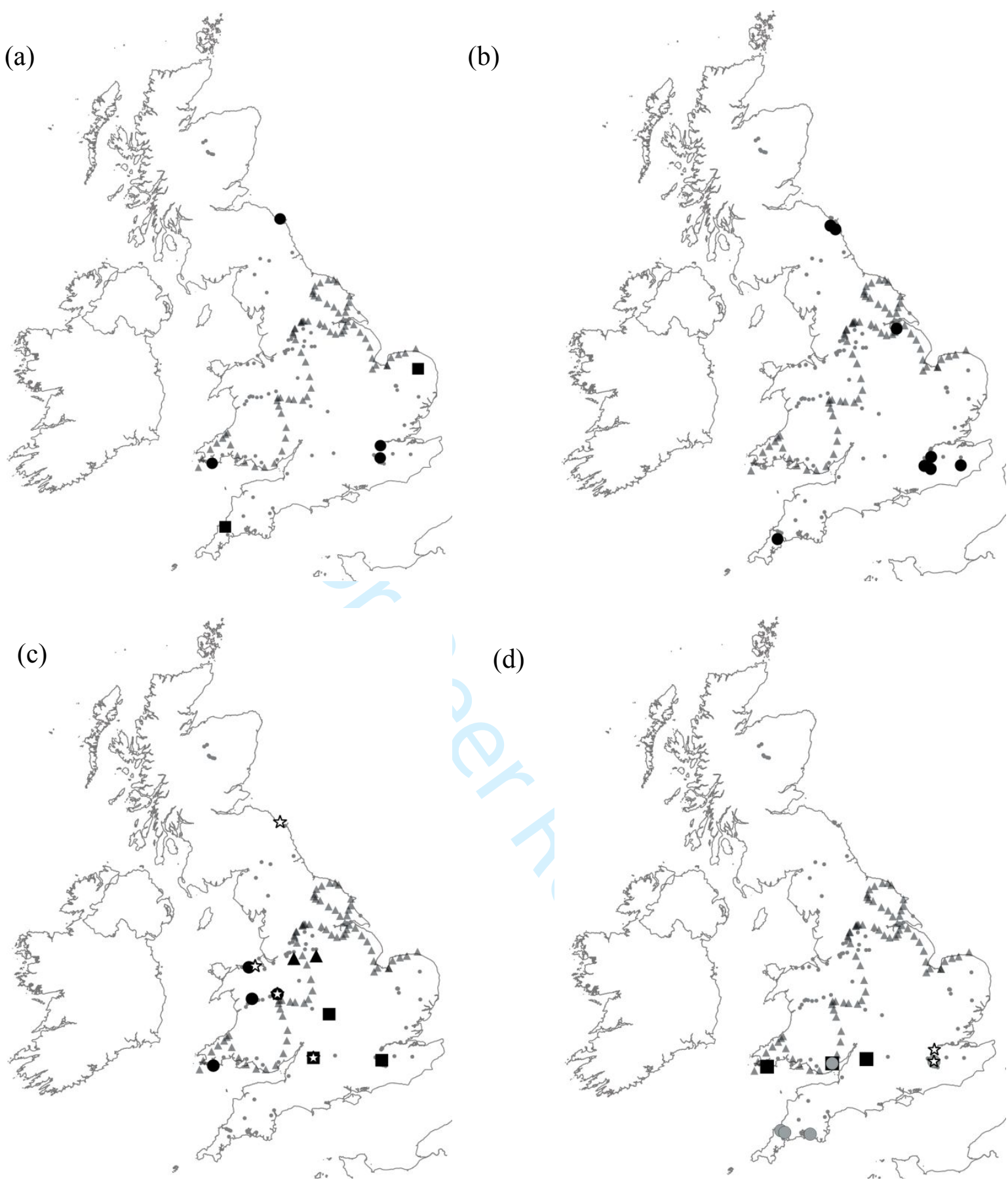

(d)

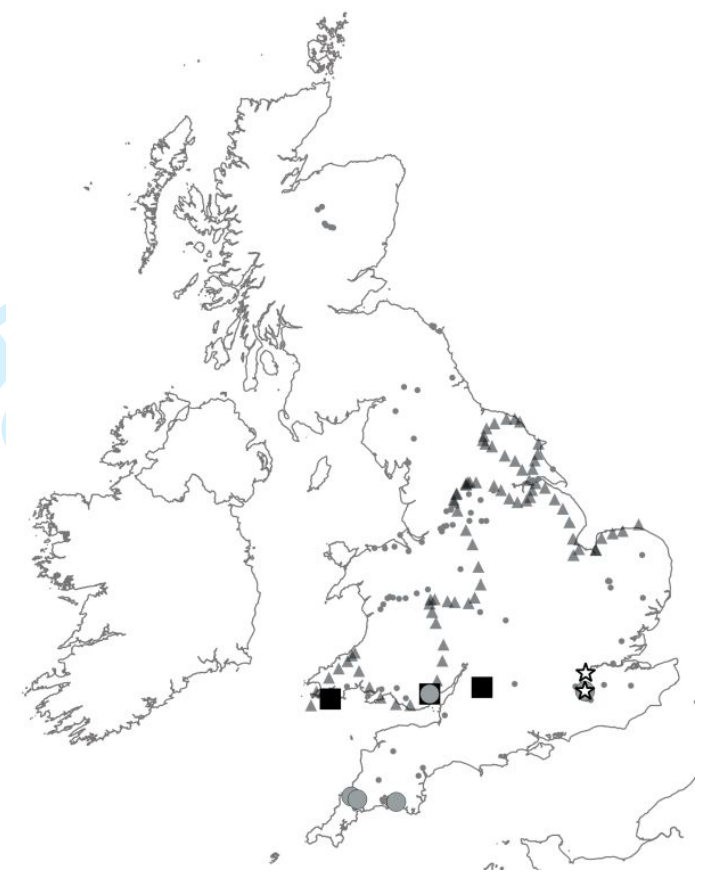

Figure 5 
Journal of Biogeography

\section{SUPPORTING INFORMATION}

\section{Evidence for the Pleistocene persistence of Collembola in Great Britain}

Christiana M. A. Faria, Peter Shaw, Brent C. Emerson

To complement the information given in the main manuscript, the following sections provide Supplementary information for material (Appendix S1), methods (Appendix S2) and results (Appendix S2-S4) used in the study

Appendix S1 Supplementary information on the material used in this study

Table S1.1. Sampling sites within the island of Great Britain, reporting the geographic coordinates and number of Entomobrya $(\mathrm{n}=722)$ and Lepidocyrtus $(\mathrm{n}=428)$ individuals collected per locality. Locations coded according to Fig 1. Sites 46, 70, 71 and 82 are absent from the list as no specimens from these genera were collected at these sites.

\begin{tabular}{ccccc}
\hline Locality & Latitude & Longitude & Entomobrya & Lepidocyrtus \\
\hline 1 & 57.22694 & -3.744723 & 3 & 2 \\
2 & 57.250828 & -3.646657 & 1 & 2 \\
3 & 57.056782 & -3.650115 & 2 & 0 \\
4 & 56.992771 & -3.484993 & 3 & 6
\end{tabular}




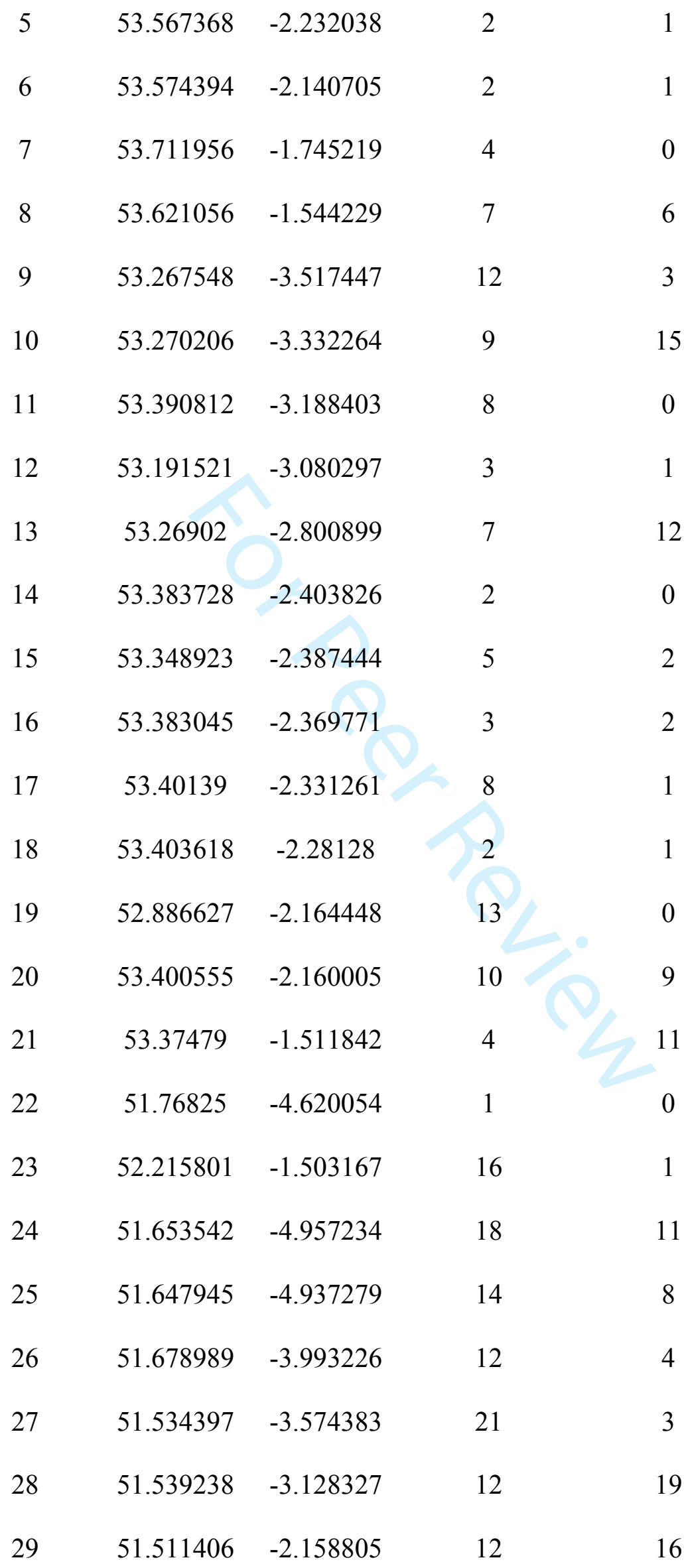




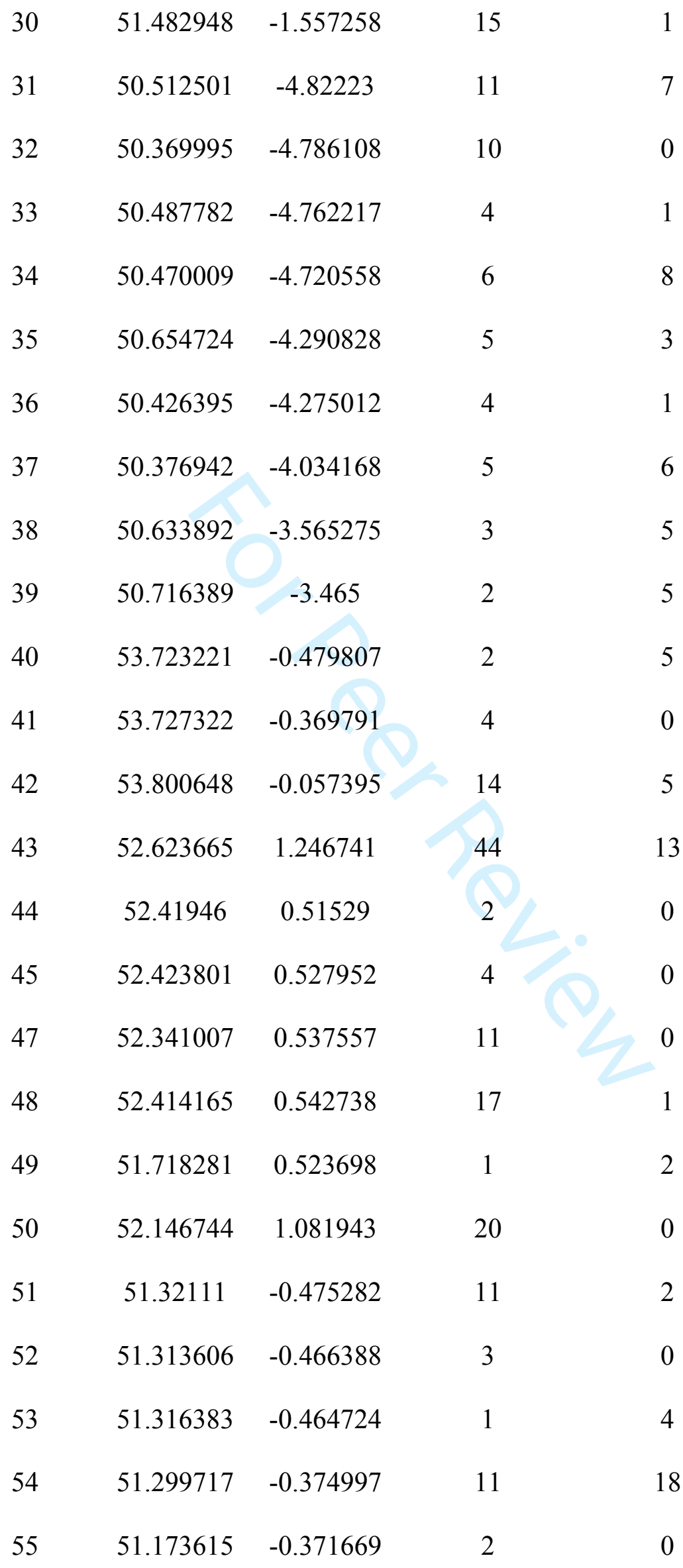




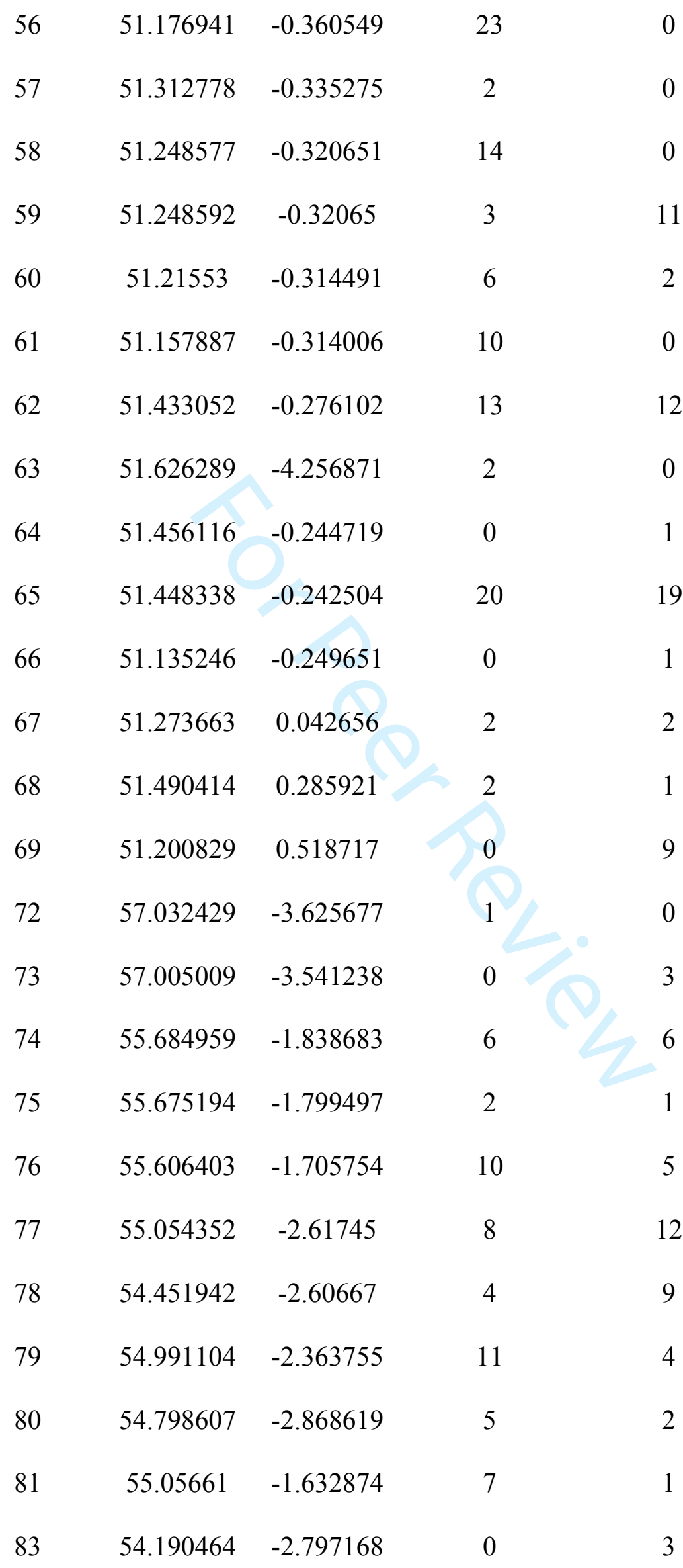




\begin{tabular}{|c|c|c|c|c|}
\hline 84 & 54.191376 & -2.795045 & 0 & 1 \\
\hline 85 & 53.498901 & -1.774891 & 6 & 4 \\
\hline 86 & 53.6982 & -1.264826 & 6 & 2 \\
\hline 87 & 53.291092 & -3.712894 & 8 & 0 \\
\hline 88 & 52.697304 & -3.519311 & 4 & 0 \\
\hline 89 & 52.699707 & -3.621308 & 3 & 10 \\
\hline 90 & 52.638248 & -3.716803 & 11 & 0 \\
\hline 91 & 52.58733 & -3.784479 & 5 & 9 \\
\hline 92 & 52.711727 & -3.575345 & 0 & 8 \\
\hline 93 & 51.269722 & -2.91999 & 1 & 4 \\
\hline 94 & 50.955917 & -3.952013 & 1 & 0 \\
\hline 95 & 51.665211 & -3.712372 & 0 & 1 \\
\hline 96 & 53.411362 & -1.830386 & 1 & 8 \\
\hline 97 & 53.383255 & -1.602855 & 12 & 7 \\
\hline 98 & 52.666122 & -3.282838 & 11 & 1 \\
\hline 99 & 52.70047 & -3.060518 & 18 & 0 \\
\hline 100 & 52.67226 & -3.407084 & 3 & \\
\hline 101 & 52.722786 & -2.839915 & 40 & 20 \\
\hline 102 & 52.362823 & -1.94556 & 13 & \\
\hline
\end{tabular}


Table S1.2. Minimum (Min), maximum (Max) and overall mean uncorrected pdistances (p-dis) found among Lepidocyrtus COII lineages collected sympatrically from six localities in Panama (sequence data from Cicconardi et al., 2013). This data was used to define the minimum pairwise distance threshold to define OTUs (see text for details).

\begin{tabular}{lcccc}
\hline Localities & N sympatric lineages & Min p-dis & Max p-dis & Overall p-dist \\
\hline L.b1b-SanFelix & 3 & 0.15 & 0.17 & 0.11 \\
L.b2b-ElValle & 3 & 0.05 & 0.17 & 0.08 \\
L.ve-Fortuna & 3 & 0.21 & 0.19 & 0.13 \\
L.ve-P.I.L.A. & 3 & 0.04 & 0.11 & 0.07 \\
L.ve-Darien & 4 & 0.04 & 0.20 & 0.17 \\
L.ve-SanFelix & 3 & 0.12 & 0.22 & 0.15 \\
\end{tabular}


Appendix S2 Supplementary material for methods and results used in this study

Table S2.1. Entomobrya and Lepidocyrtus monophyletic clusters recovered using the following approaches: NJ full - Neighbour-Joining with all haplotypes dataset, BI hap - Bayesian inference with unique haplotypes dataset, ML hap - Maximum Likelihood with unique haplotypes dataset, ML full - Maximum Likelihood with all haplotypes dataset (see text for details). Posterior (max. 1) and bootstrap (max. 100) support values are presented for BI and NJ/ML trees respectively. Symbol $(\checkmark)$ denotes a cluster has been recovered. Singleton indicates clusters defined by a single haplotype sequence, no support value associated. Variations in cluster recovering (e.g. split or association with other clusters) are indicated. For example, within Entomobrya cluster 4 two monophyletic clusters NIV1 and NIV2 can be found.

\begin{tabular}{|c|c|c|c|c|c|c|c|c|}
\hline \multicolumn{9}{|c|}{ ENTOMOBRYA DATASET } \\
\hline Cluster & NJ full tree & bootstrap & BI hap tree & posterior & ML hap tree & bootstrap & ML full tree & bootstrap \\
\hline 1 & $\checkmark$ & 99 & $\checkmark$ & 1 & $\checkmark$ & 83 & $\checkmark$ & 80 \\
\hline 2 & $\checkmark$ & 99 & $\checkmark$ & singleton & $\checkmark$ & singleton & $\checkmark$ & 97 \\
\hline 3 & $\checkmark$ & 99 & $\checkmark$ & 1 & $\checkmark$ & 100 & $\checkmark$ & 98 \\
\hline 4 & $\begin{array}{c}\checkmark \text { with } \\
\text { Niv1, Niv2 }\end{array}$ & 99 & $\begin{array}{c}\checkmark \text { with } \\
\text { Niv1, Niv2 }\end{array}$ & 1 & $\begin{array}{c}\checkmark \text { with Niv1 but } \\
\text { not Niv2 }\end{array}$ & 51 & $\begin{array}{c}\checkmark \text { with Niv1 but } \\
\text { not Niv2 }\end{array}$ & 67 \\
\hline 5 & $\checkmark$ & 99 & $\checkmark$ & 1 & $\checkmark$ & 100 & $\checkmark$ & 97 \\
\hline 6 & $\checkmark$ & 99 & $\checkmark$ & 1 & $\checkmark$ & 100 & $\checkmark$ & 98 \\
\hline 7 & $\checkmark$ & singleton & $\checkmark$ & singleton & $\checkmark$ & singleton & $\checkmark$ & singleton \\
\hline 8 & $\checkmark$ & 99 & $\checkmark$ & 1 & $\checkmark$ & 94 & $\checkmark$ & 97 \\
\hline 9 & $\checkmark$ & 99 & $\checkmark$ & 1 & $\checkmark$ & 97 & $\checkmark$ & 96 \\
\hline 10 & $\checkmark$ & singleton & $\checkmark$ & singleton & $\checkmark$ & singleton & $\checkmark$ & singleton \\
\hline 11 & $\checkmark$ & singleton & $\checkmark$ & singleton & $\checkmark$ & singleton & $\checkmark$ & singleton \\
\hline 12 & $\checkmark$ & 99 & $\checkmark$ & 1 & $\checkmark$ & 92 & $\checkmark$ & 89 \\
\hline 13 & $\checkmark$ & 99 & $\checkmark$ & 1 & $\checkmark$ & 91 & $\checkmark$ & 93 \\
\hline \multicolumn{9}{|c|}{ LEPIDOCYRTUS DATASET } \\
\hline
\end{tabular}




\begin{tabular}{|c|c|c|c|c|c|c|c|c|}
\hline Cluster & NJ full tree & bootstrap & BI tree hap & posterior & ML tree hap & bootstrap & ML full tree & bootstrap \\
\hline 1 & $\checkmark$ & 100 & $\checkmark$ & 1 & $\checkmark$ & 97 & $\checkmark$ & 67 \\
\hline 2 & $\checkmark$ & 100 & $\checkmark$ & singleton & $\checkmark$ & singleton & $\checkmark$ & 98 \\
\hline 3 & $\checkmark$ & 100 & $\checkmark$ & 1 & $\checkmark$ & 100 & $\checkmark$ & 95 \\
\hline 4 & $\checkmark$ & singleton & $\checkmark$ & singleton & $\checkmark$ & singleton & $\checkmark$ & singleton \\
\hline 5 & $\checkmark$ & singleton & $\checkmark$ & singleton & $\checkmark$ & singleton & $\checkmark$ & singleton \\
\hline 6 & $\checkmark$ & 100 & $\checkmark$ & 1 & $\checkmark$ & 100 & $\checkmark$ & 91 \\
\hline 7 & split in 2 & 100 & $\checkmark$ & 1 & $\checkmark$ & 100 & $\checkmark$ & 90 \\
\hline 8 & $\checkmark$ & 100 & $\checkmark$ & 1 & no & within 9 & no & within 9 \\
\hline 9 & split in 2 & 99 & $\checkmark$ & 1 & no split, plus 8 & 97 & no split, plus 8 & 94 \\
\hline 10 & $\checkmark$ & singleton & $\checkmark$ & singleton & $\checkmark$ & singleton & $\checkmark$ & singleton \\
\hline 11 & $\checkmark$ & 100 & $\checkmark$ & 1 & $\checkmark$ & 99 & $\checkmark$ & 91 \\
\hline 12 & $\checkmark$ & 100 & $\checkmark$ & 1 & $\checkmark$ & 98 & $\checkmark$ & 92 \\
\hline 13 & $\checkmark$ & 100 & $\checkmark$ & 0.99 & $\checkmark$ & 99 & $\checkmark$ & 94 \\
\hline 14 & $\checkmark$ & singleton & $\checkmark$ & singleton & $\checkmark$ & singleton & $\checkmark$ & singleton \\
\hline 15 & split in 2 & 100 & $\checkmark$ & 1 & $\checkmark$ & 98 & $\checkmark$ & 44 \\
\hline 16 & $\checkmark$ & 100 & $\checkmark$ & 1 & $\checkmark$ & 1 & $\checkmark$ & 96 \\
\hline 17 & $\checkmark$ & 100 & $\checkmark$ & 1 & $\checkmark$ & 1 & $\checkmark$ & 89 \\
\hline 18 & $\checkmark$ & 100 & $\checkmark$ & 1 & $\checkmark$ & 87 & $\checkmark$ & 85 \\
\hline
\end{tabular}


Table S2.2 Delimitation of Entomobrya and Lepidocyrtus OTUs using the following approaches: 96\% threshold analysis, GMYC, mPTP using BI unique haplotypes tree (BI hap), mPTP using ML unique haplotypes tree (ML hap) and MPTP using ML all haplotypes tree (ML full) (see text for details). Symbol $(\checkmark)$ denotes that OTU has been defined by that method. Variations in delimited OTUs (such as splits or combinations) are also indicated. For example, GMYC splits Entomobrya OTU1 in four different entities. N recovered counts the number of times an OTU (without variations) has been delimited considering these five approaches. Final numbers of OTUs for each genus consider the most frequent "form" of OTU while conservatively avoiding over splitting. For example, Entomobrya OTU1 without splits is defined in 4 out of 5 approaches, thus it is considered a single OTU. Entomobrya OTU10 is combined with OTU11 in 3 out of 5 approaches, thus both are considered a single OTU. Lepidocyrtus OTU9 is trickier because it has been split in 2 approaches and combined with OTU8 in other 2 approaches. We kept it as a single OTU because OTU8 is defined in the majority of approaches ( 3 out of 5), then, when looking at only OTU9, it appears without splits in 3 out of 5 approaches, thus being considered a single OTU.

\begin{tabular}{ccccccc}
\hline \multicolumn{7}{c}{ ENTOMOBRY DATASET } \\
\hline \multirow{2}{*}{ OTU } & $\begin{array}{c}\mathbf{9 6 \%} \\
\text { threshold }\end{array}$ & $\begin{array}{c}\text { GMYC } \\
\text { (BI hap) }\end{array}$ & $\begin{array}{c}\text { mPTP } \\
\text { (BI hap) }\end{array}$ & $\begin{array}{c}\text { mPTP } \\
\text { (ML hap) }\end{array}$ & $\begin{array}{c}\text { mPTP } \\
\text { (ML full) }\end{array}$ & N recovered \\
\hline 1 & $\checkmark$ & split in 4 & $\checkmark$ & $\checkmark$ & $\checkmark$ & 4 \\
2 & $\checkmark$ & $\checkmark$ & $\checkmark$ & $\checkmark$ & $\checkmark$ & 5 \\
3 & $\checkmark$ & $\checkmark$ & $\checkmark$ & $\checkmark$ & $\checkmark$ & 5 \\
4 & $\checkmark$ & split in 5 & split in 2 & $\checkmark$ & $\checkmark$ & 3 \\
5 & $\checkmark$ & $\checkmark$ & $\checkmark$ & $\checkmark$ & $\checkmark$ & 5 \\
6 & $\checkmark$ & $\checkmark$ & $\checkmark$ & $\checkmark$ & $\checkmark$ & 5 \\
7 & $\checkmark$ & $\checkmark$ & plus 9 & $\checkmark$ & $\checkmark$ & 4 \\
8 & $\checkmark$ & $\checkmark$ & $\checkmark$ & $\checkmark$ & $\checkmark$ & 5 \\
9 & $\checkmark$ & split in 2 & plus 7 & $\checkmark$ & $\checkmark$ & 3 \\
10 & $\checkmark$ & $\checkmark$ & plus 11 & plus 11 & plus 11 & 2 \\
11 & $\checkmark$ & $\checkmark$ & plus 10 & plus 10 & plus 10 & 2 \\
12 & $\checkmark$ & $\checkmark$ & $\checkmark$ & $\checkmark$ & $\checkmark$ & 5 \\
13 & $\checkmark$ & split in 2 & split in 2 & $\checkmark$ & $\checkmark$ & 3 \\
Total & 13 & 22 & 13 & 12 & 12 & $\mathbf{1 2}$
\end{tabular}




\begin{tabular}{|c|c|c|c|c|c|c|}
\hline \multicolumn{7}{|c|}{ LEPIDOCYRTUS DATASET } \\
\hline OTU & $\begin{array}{c}96 \% \\
\text { threshold }\end{array}$ & $\begin{array}{c}\text { GMYC } \\
\text { (BI hap) }\end{array}$ & $\begin{array}{c}\text { mPTP } \\
\text { (BI hap) }\end{array}$ & $\begin{array}{c}\text { mPTP } \\
\text { (ML hap) }\end{array}$ & $\begin{array}{c}\text { mPTP } \\
\text { (ML full) }\end{array}$ & $\mathbf{N}$ recovered \\
\hline 1 & $\checkmark$ & $\checkmark$ & $\checkmark$ & $\checkmark$ & $\checkmark$ & 5 \\
\hline 2 & $\checkmark$ & $\checkmark$ & $\checkmark$ & $\checkmark$ & $\checkmark$ & 5 \\
\hline 3 & $\checkmark$ & $\checkmark$ & $\checkmark$ & $\checkmark$ & $\checkmark$ & 5 \\
\hline 4 & $\checkmark$ & $\checkmark$ & $\checkmark$ & $\checkmark$ & $\checkmark$ & 5 \\
\hline 5 & $\checkmark$ & $\checkmark$ & $\checkmark$ & $\checkmark$ & $\checkmark$ & 5 \\
\hline 6 & $\checkmark$ & $\checkmark$ & $\checkmark$ & $\checkmark$ & $\checkmark$ & 5 \\
\hline 7 & split in 2 & $\checkmark$ & split in 2 & $\checkmark$ & $\checkmark$ & 3 \\
\hline 8 & $\checkmark$ & $\checkmark$ & $\checkmark$ & within 9 & within 9 & 3 \\
\hline 9 & split in 2 & $\checkmark$ & split in 2 & $\checkmark$ plus 8 & $\checkmark$ plus 8 & 1 \\
\hline 10 & $\checkmark$ & $\checkmark$ & $\checkmark$ & $\checkmark$ & $\checkmark$ & 5 \\
\hline 11 & $\checkmark$ & $\checkmark$ & $\checkmark$ & $\checkmark$ & $\checkmark$ & 5 \\
\hline 12 & $\checkmark$ & $\checkmark$ & $\checkmark$ & $\checkmark$ & $\checkmark$ & 5 \\
\hline 13 & $\checkmark$ & $\checkmark$ & $\checkmark$ & $\checkmark$ & $\checkmark$ & 5 \\
\hline 14 & $\checkmark$ & $\checkmark$ & $\checkmark$ & $\checkmark$ & $\checkmark$ & 5 \\
\hline 15 & split in 3 & $\checkmark$ & split in 2 & $\checkmark$ & split in 2 & 2 \\
\hline 16 & $\checkmark$ & $\checkmark$ & $\checkmark$ & $\checkmark$ & $\checkmark$ & 5 \\
\hline 17 & $\checkmark$ & $\checkmark$ & $\checkmark$ & $\checkmark$ & $\checkmark$ & 5 \\
\hline 18 & $\checkmark$ & $\checkmark$ & split in 2 & $\checkmark$ & $\checkmark$ & 4 \\
\hline Total & 22 & 18 & 22 & 17 & 18 & 18 \\
\hline
\end{tabular}


Figure S2.1. Maximum likelihood tree of Entomobrya mtCOI Sanger sequences (using all haplotypes) derived from 90 sampling sites across Great Britain (see Fig. 1, Appendix S1: Table S1.1). Katianna sp. was sampled as an outgroup. Numbers immediately to the right of morphospecies names correspond to sampling sites. A monophyletic cluster of DNA sequences, NivG1, is grey highlighted, which represents a clear structuring of genetic variation, found within OTU 4. Further OTU details can be found in Table 1 . 
Figure S2.2. Maximum likelihood tree of Lepidocyrtus mtCOI Sanger sequences (using all haplotypes) derived from 68 sampling sites across Great Britain (see Fig. 1, Appendix S1: Table S1.1). Katianna sp. was sampled as an outgroup. Numbers immediately to the right of morphospecies names correspond to sampling sites. Geographically localized OTUs are grey highlighted, except OTUs 6 and 16 because they did not present sequence variation. Further OTU details can be found in Table 1. 
Appendix S3 Spatial distribution patterns for Entomobrya and Lepidocyrtus OTUs

(a)

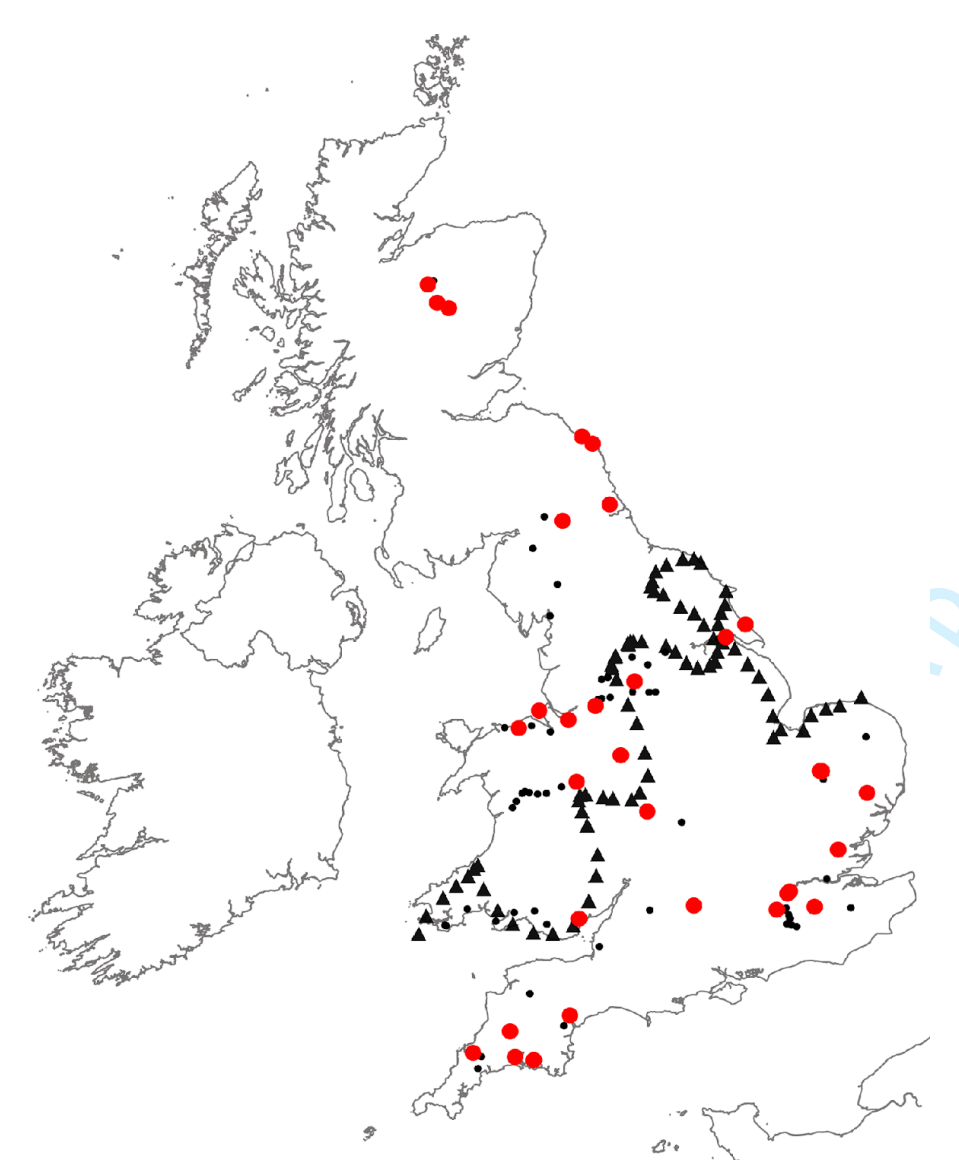

(b)

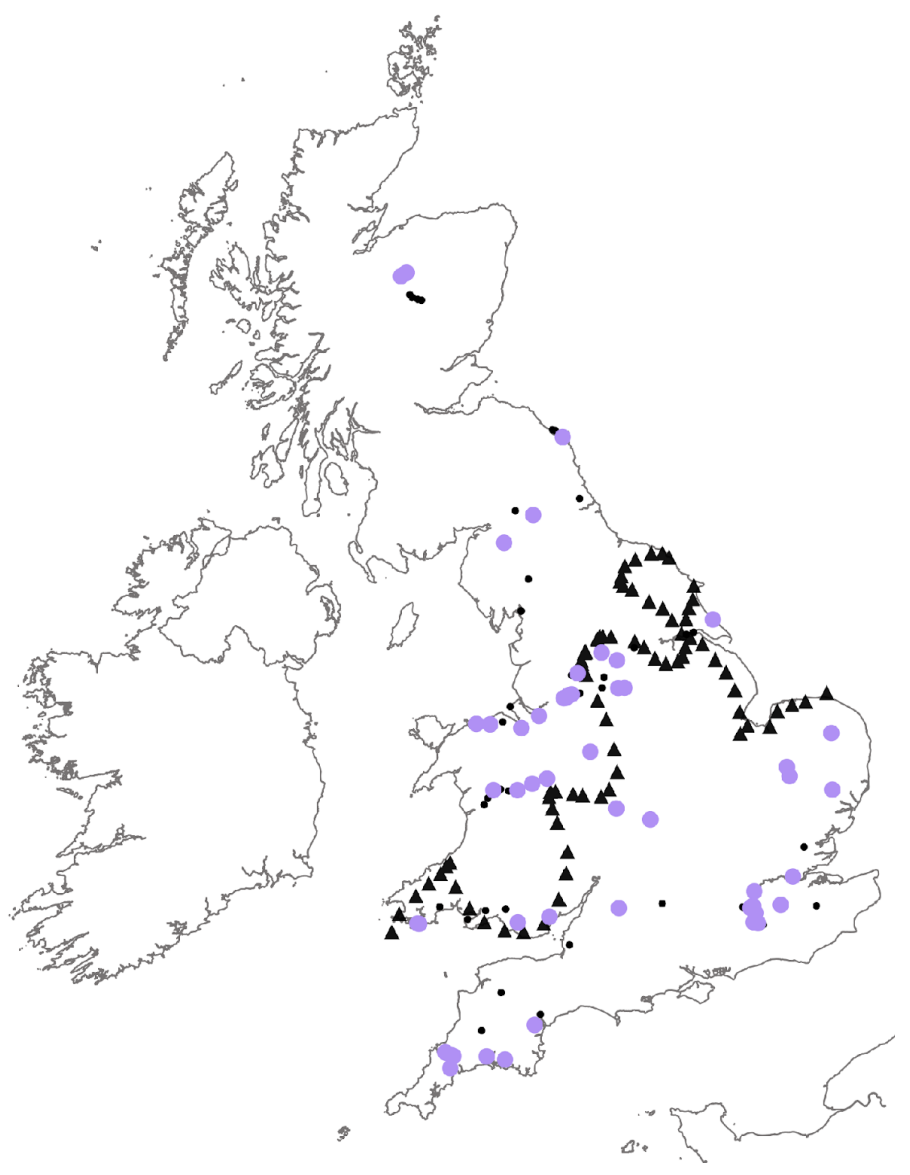

Figure S3.1. Geographical distributions of Entomobrya a) OTU 11 - red dots, and b) OTU 12 - purple dots, according to their sampling localities in Great Britian. Small black dots indicate remaining sampling sites. Black triangles indicate the maximum extent of the British-Irish ice sheet during the last Pleistocene glacial period. 
(a)

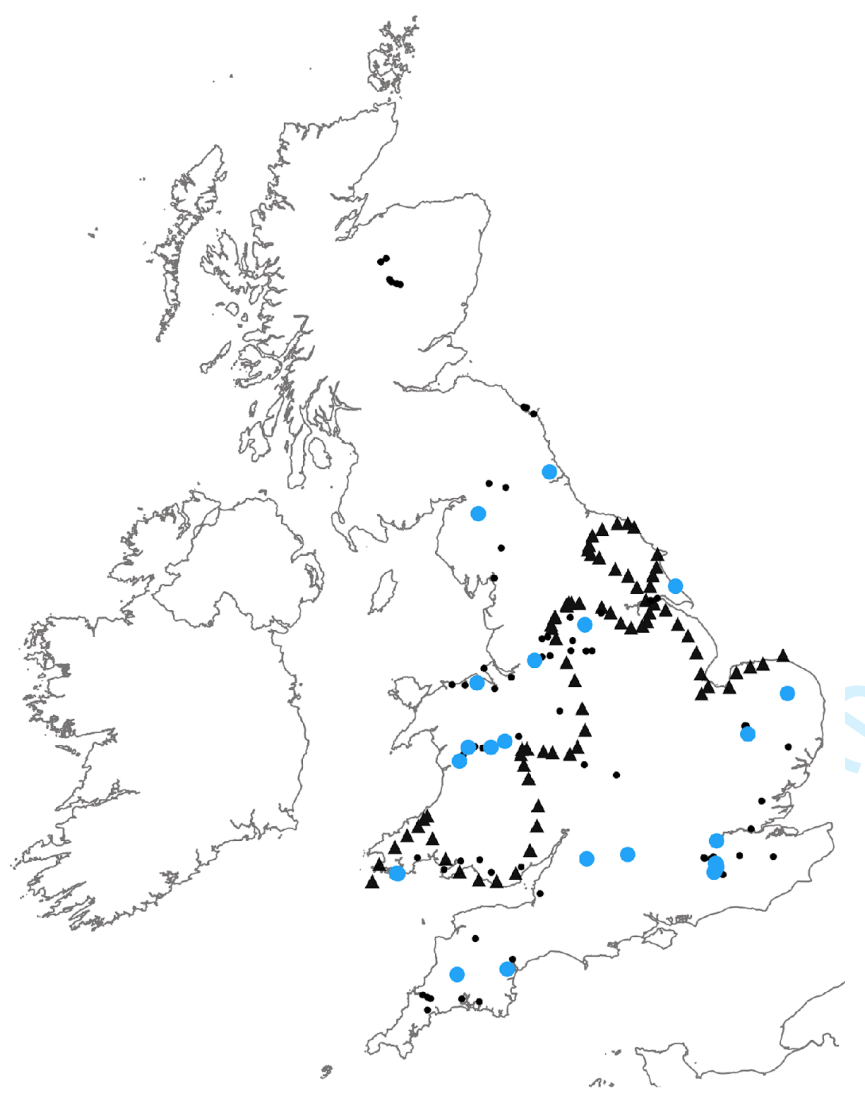

(b)

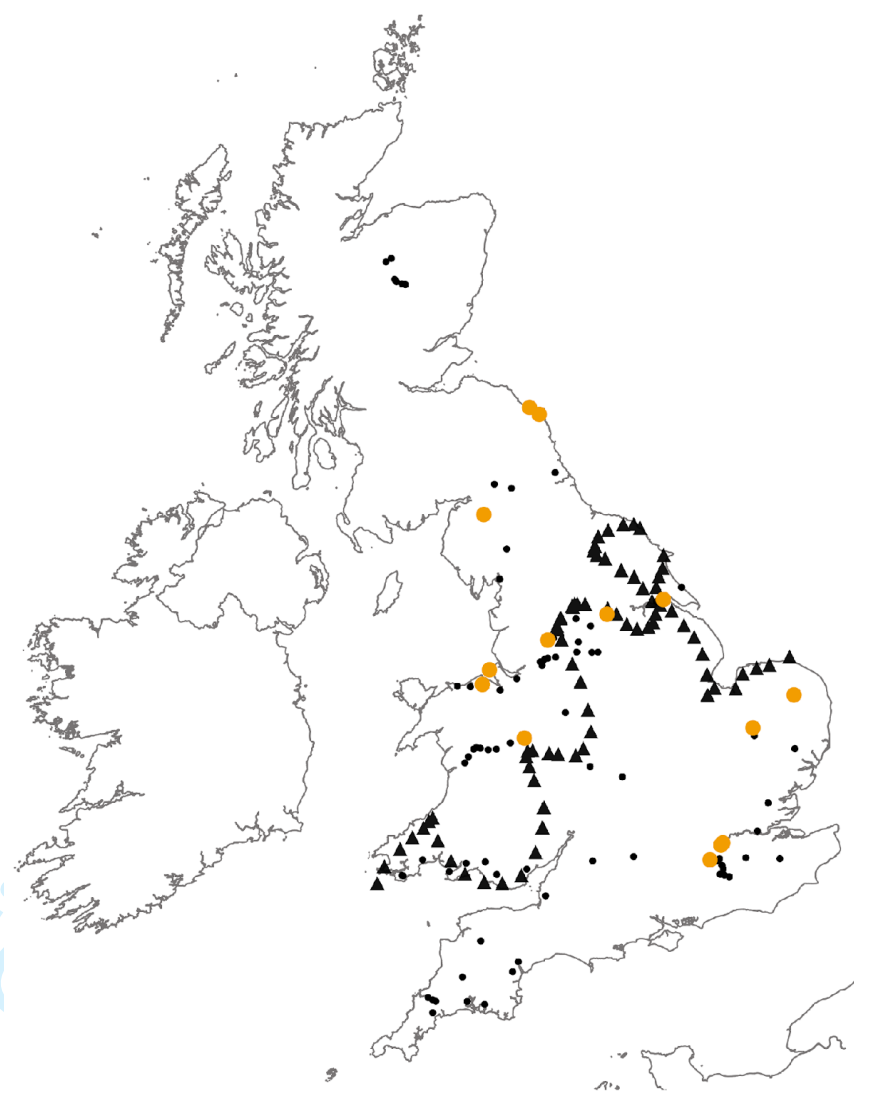

Figure S3.2. Geographical distributions of Entomobrya a) OTU 5 - blue dots, and b) OTU 6 - orange dots, according to their sampling localities in Great Britain. Small black dots indicate remaining sampling sites. Black triangles indicate the maximum extent of the British-Irish ice sheet during the last Pleistocene glacial period. 


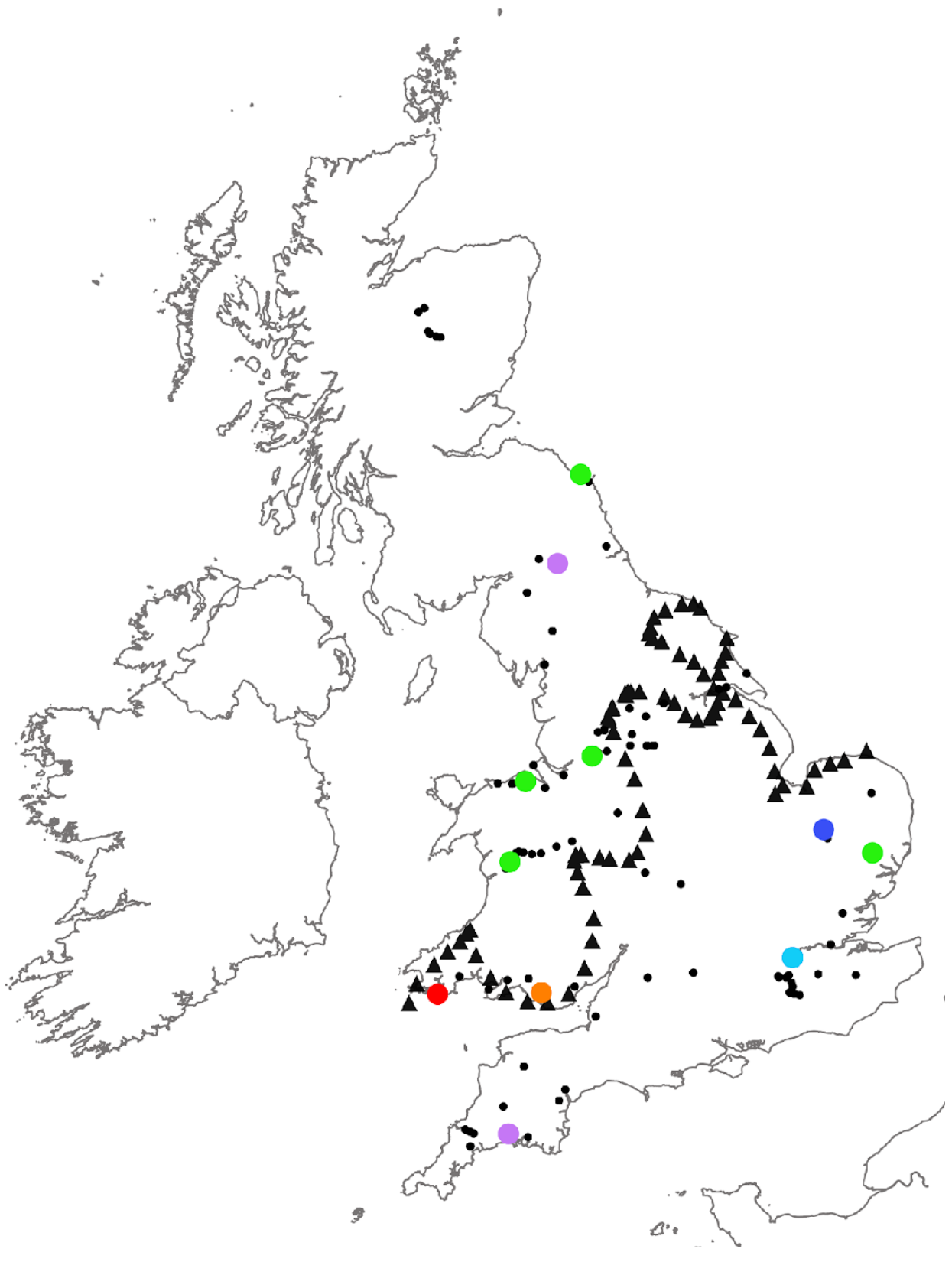

Figure S3.3. Geographical distributions of Entomobrya mtDNA COI OTUs according to their sampling localities in Great Britain. OTU 2 - green dots, OTU 3 - purple dots, OTU 7 - light blue dot, OTU 8 - orange dot, OTU 9 - dark and light blue dots, OTU 10 - light blue and red dots. Black dots indicate remaining sampling sites. Black triangles indicate the maximum extent of the British-Irish ice sheet during the last Pleistocene glacial period. 
(a)

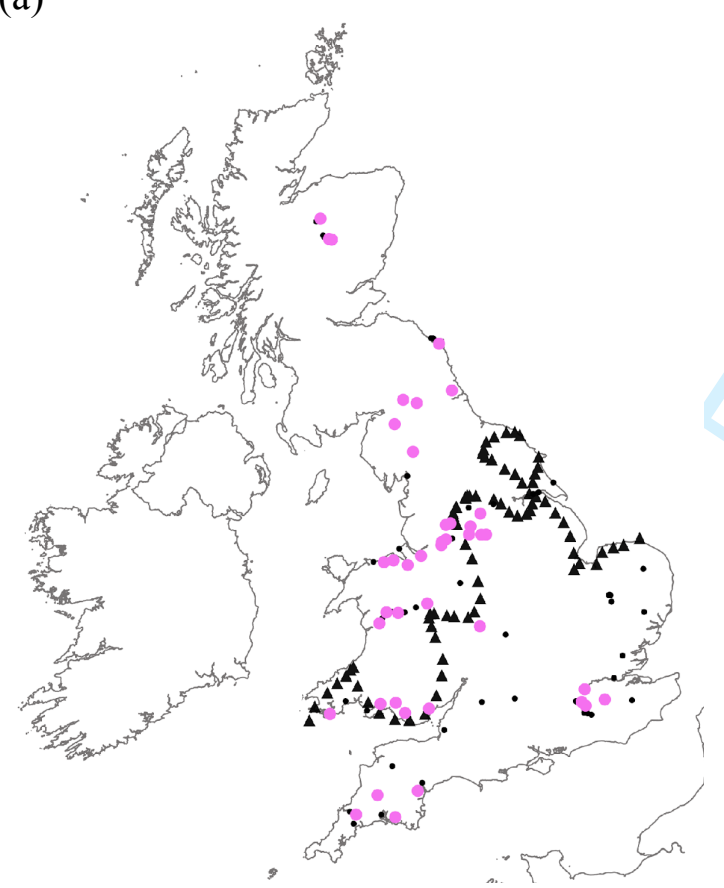

(b)

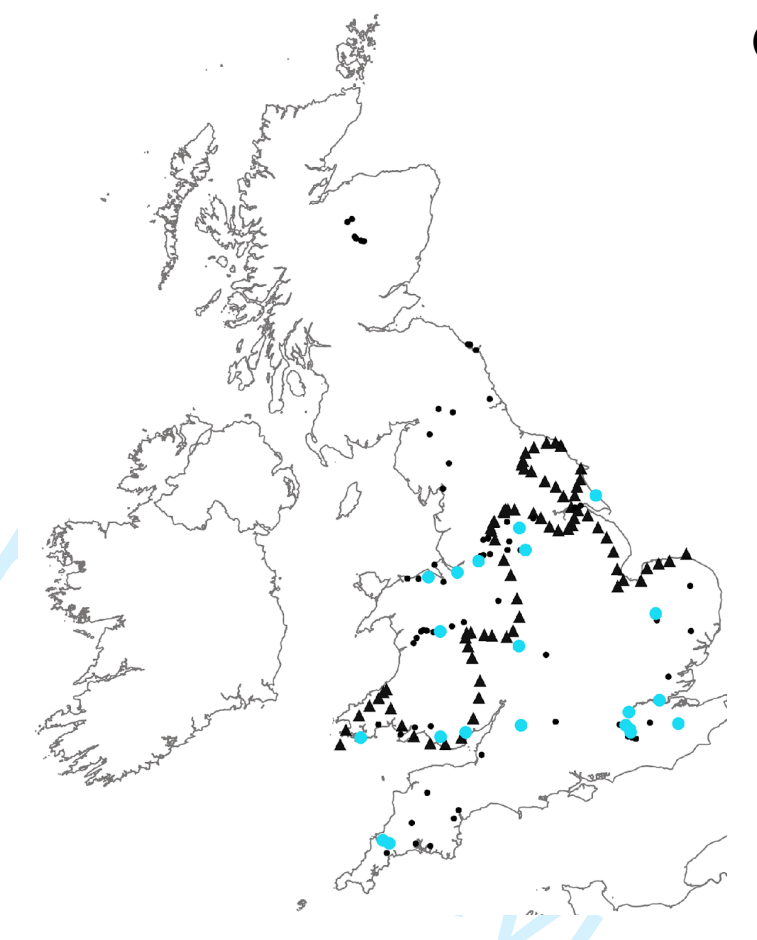

(c)

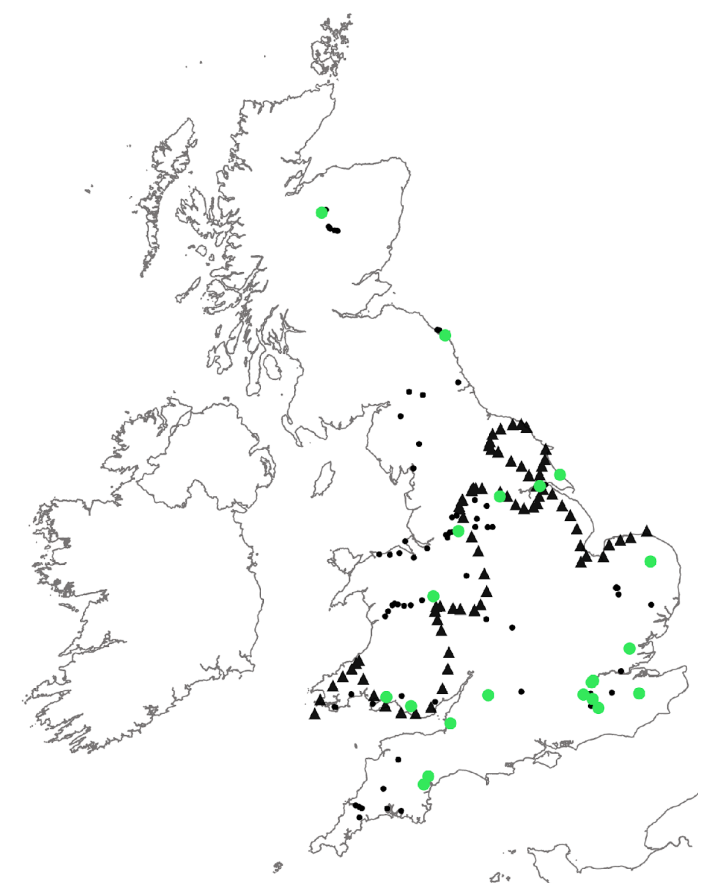

Figure S3.4. Geographical distributions of widespread Lepidocyrtus in Great Britain a) OTU 1 - pink dots, b) OTU 9 - blue dots, c) OTU 15 - green dots. Small black dots indicate remaining sampling sites. Black triangles indicate the maximum extent of the British-Irish ice sheet during the last Pleistocene glacial period. 
(a)

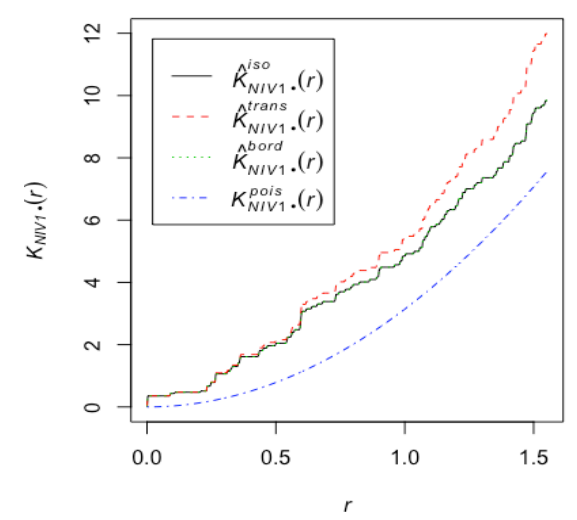

(c)

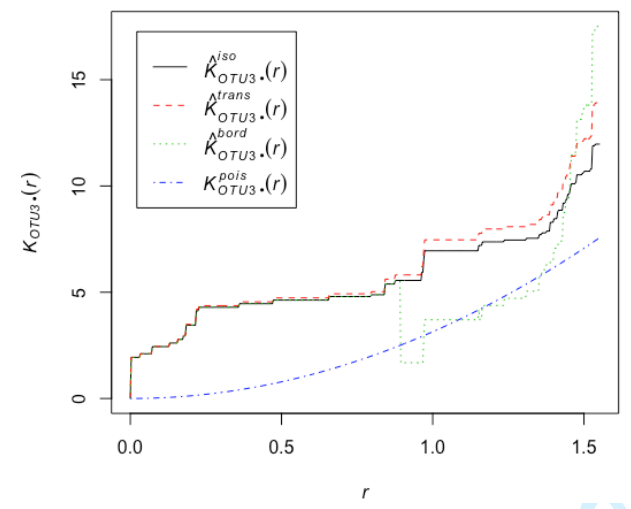

(e)

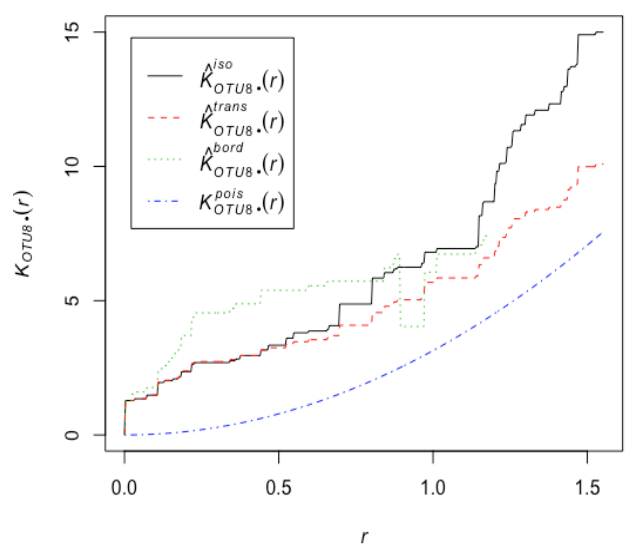

(g)

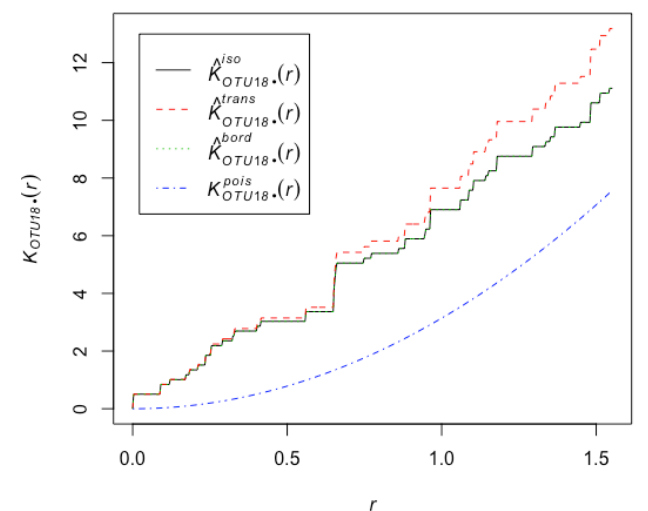

(b)

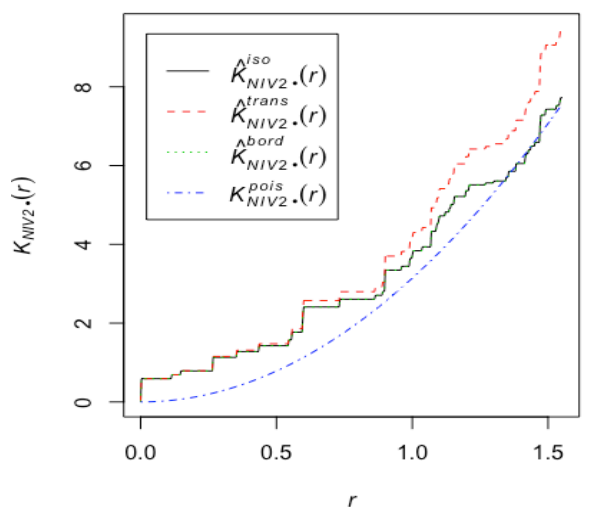

(d)

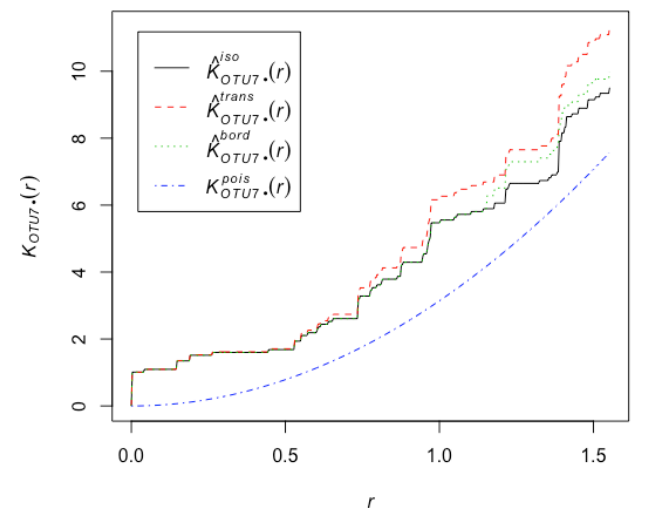

(f)

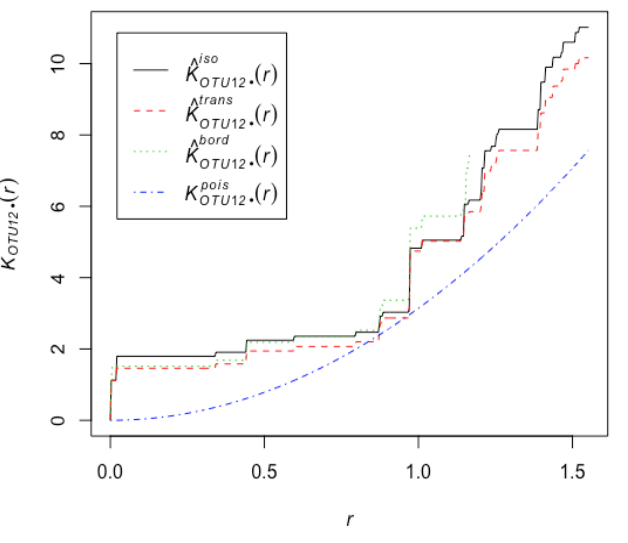


Figure S3.5. Ripley's Kdot function for spatial distribution patterns for Entomobrya and Lepidocyrtus localized monophyletic clusters sampled in Great Britain. Plots show that OTUs deviate from completely random distribution with empirical values (black, red and green lines) greater than that expected for a random distribution (blue dashed lines), indicating their distribution to be clustered. $\mathrm{K}_{\text {iso }}$ - isotropic correction, $\mathrm{K}_{\text {trans }}$ - translation correction, $\mathrm{K}_{\text {bord }}$ - border method or reduced samples estimator, $\mathrm{K}_{\mathrm{pois}}$ - completely random Poisson point process. Localized monophyletic clusters: Entomobrya OTU4 a) NIVG1, b) NIVG2, Lepidocyrtus c) OTU3, d) OTU7, e) OTU8, f) OTU12, g) OTU18. 
(a)

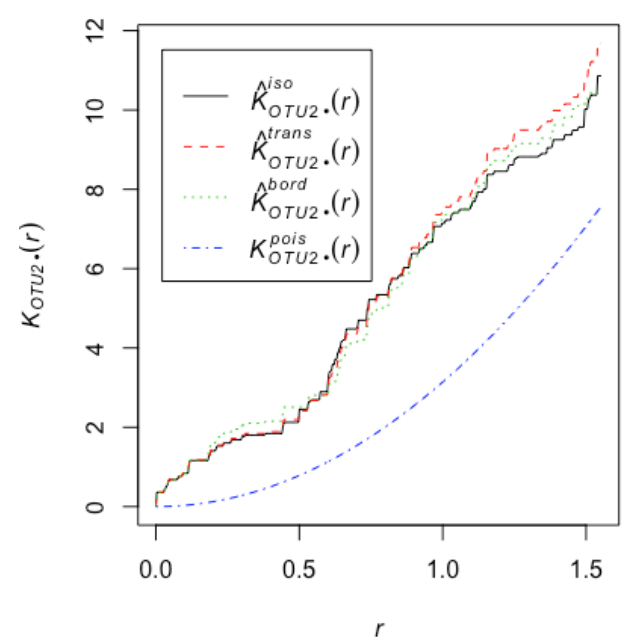

(c)

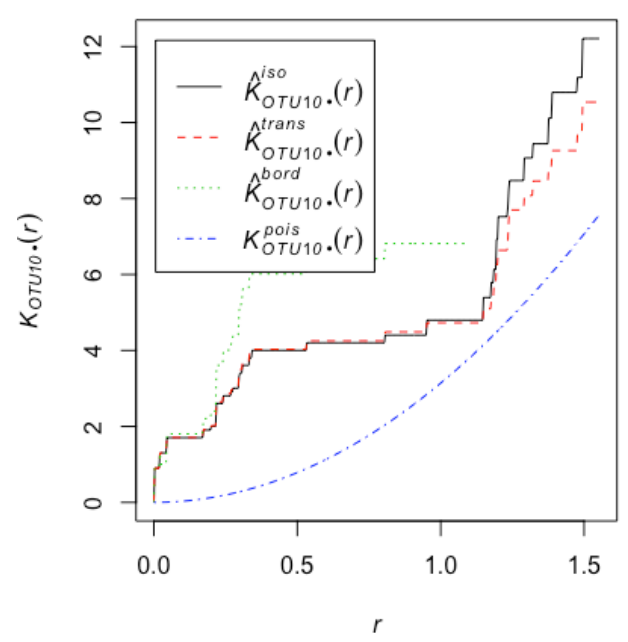

(e)

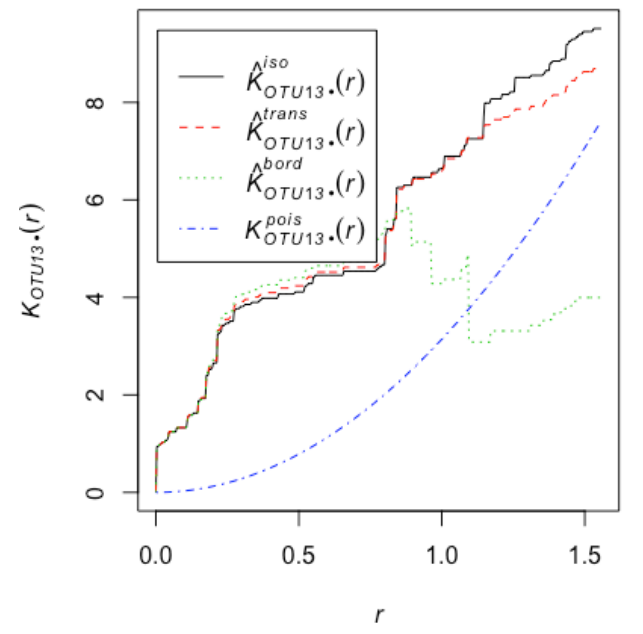

(b)

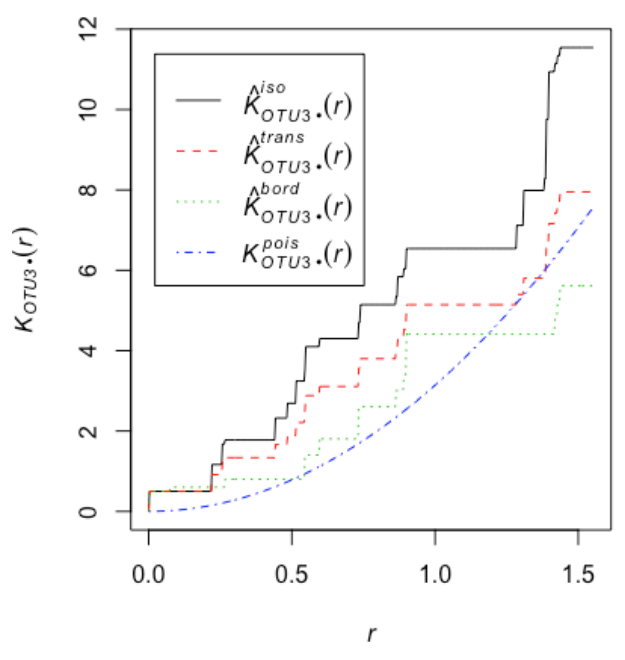

(d)

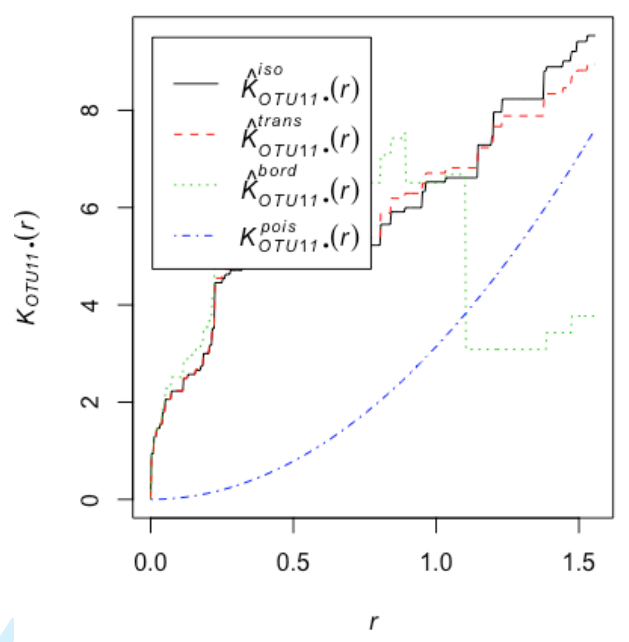

(f)

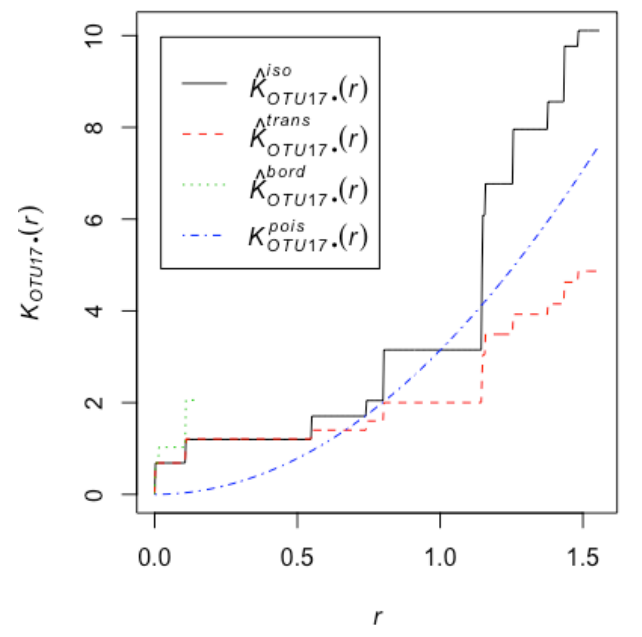


Figure S3.6. Ripley's Kdot function for spatial distribution patterns for Entomobrya and Lepidocyrtus disjunctly distributed clusters sampled in Great Britain. Plots show that OTUs mostly deviate from completely random distribution with empirical values (black, red and green lines) greater than that expected for a random distribution (blue dashed lines). $\mathrm{K}_{\text {iso }}-$ isotropic correction, $\mathrm{K}_{\text {trans }}$ - translation correction, $\mathrm{K}_{\text {bord }}$ - border method or reduced samples estimator, $\mathrm{K}_{\text {pois }}$ - completely random Poisson point process. Disjunctly distributed clusters: Entomobrya a) OTU2, b) OTU3, c) OTU10 and Lepidocyrtus d) OTU11, e) OTU13, f) OTU17. 
Appendix S4 Dated trees for Entomobrya and Lepidocyrtus geographically localized monophyletic clusters

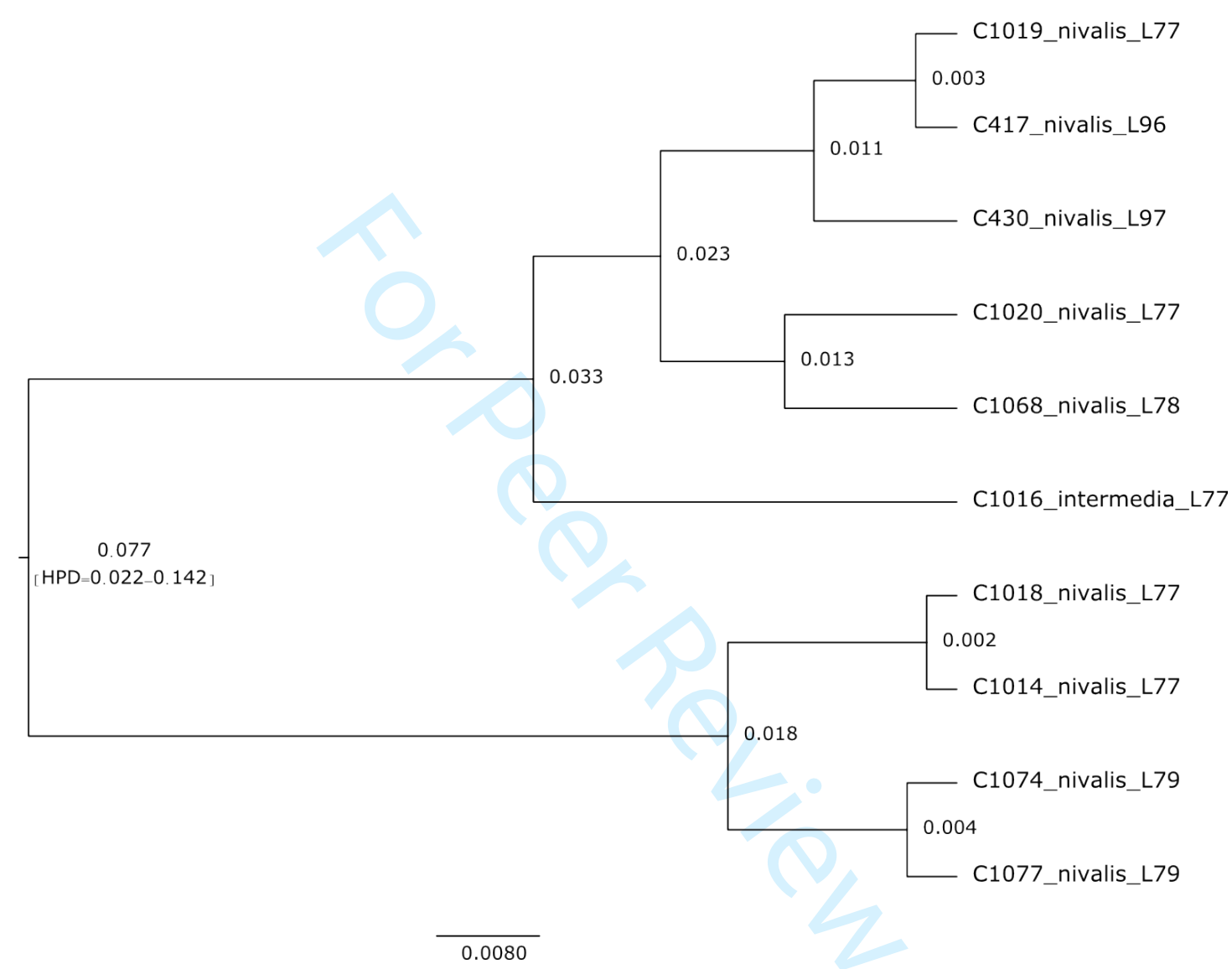

Figure S4.1. BEAST tree for Entomobrya geographically localized monophyletic cluster NIVG1 found within the widespread OTU4. Numbers after the name indicate localities where sequences were sampled in Great Britain (Table S1). Node ages were estimated using a Collembola COI rate of 0.0421 substitutions/site/Ma (see text for details). 


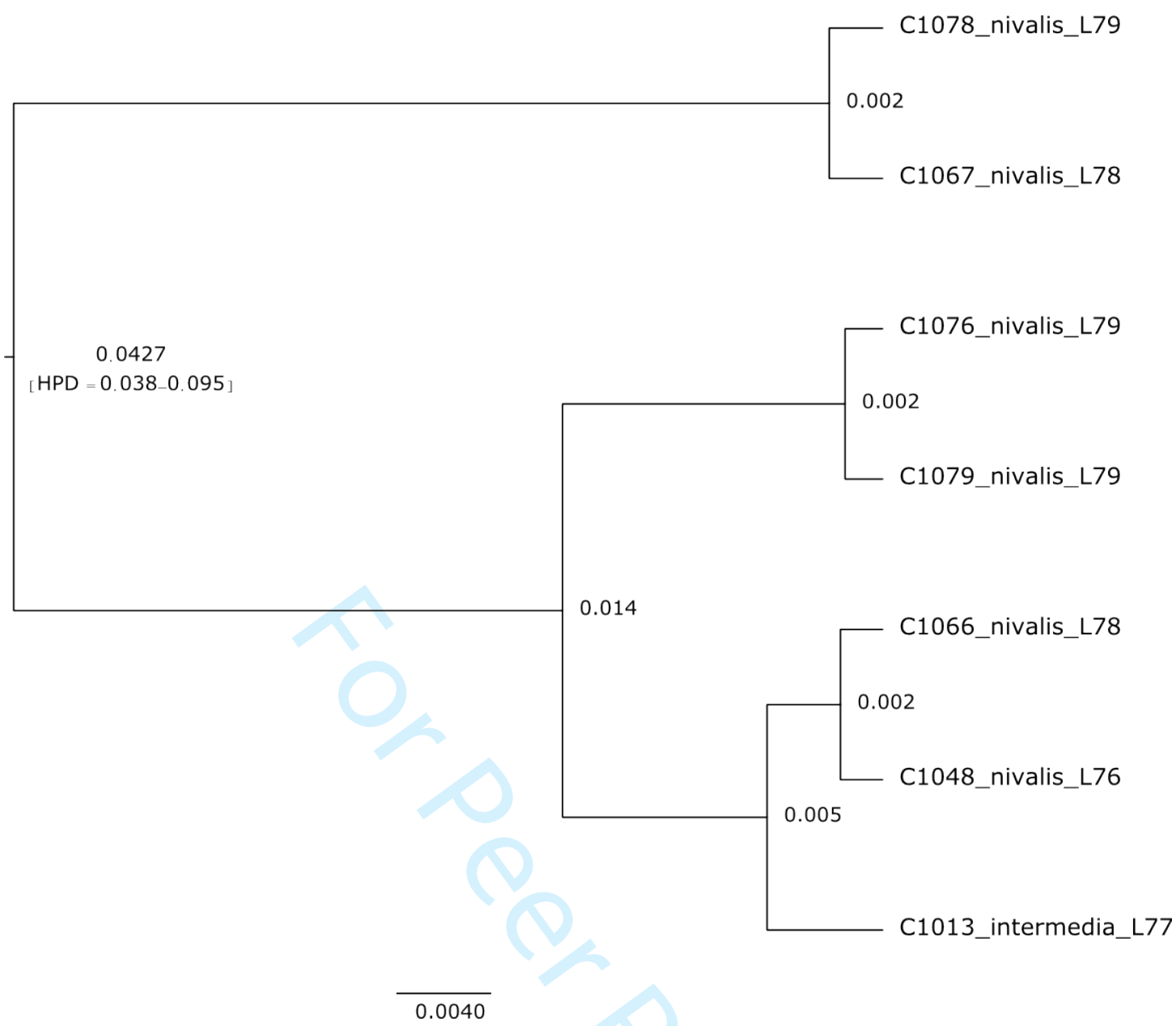

Figure S4.2. BEAST tree for Entomobrya geographically localized monophyletic cluster NIVG2 found within the widespread OTU4. Numbers after the name indicate localities where sequences were sampled in Great Britain (Table S1). Node ages were estimated using a Collembola COI rate of 0.0421 substitutions/site/Ma (see text for details). 


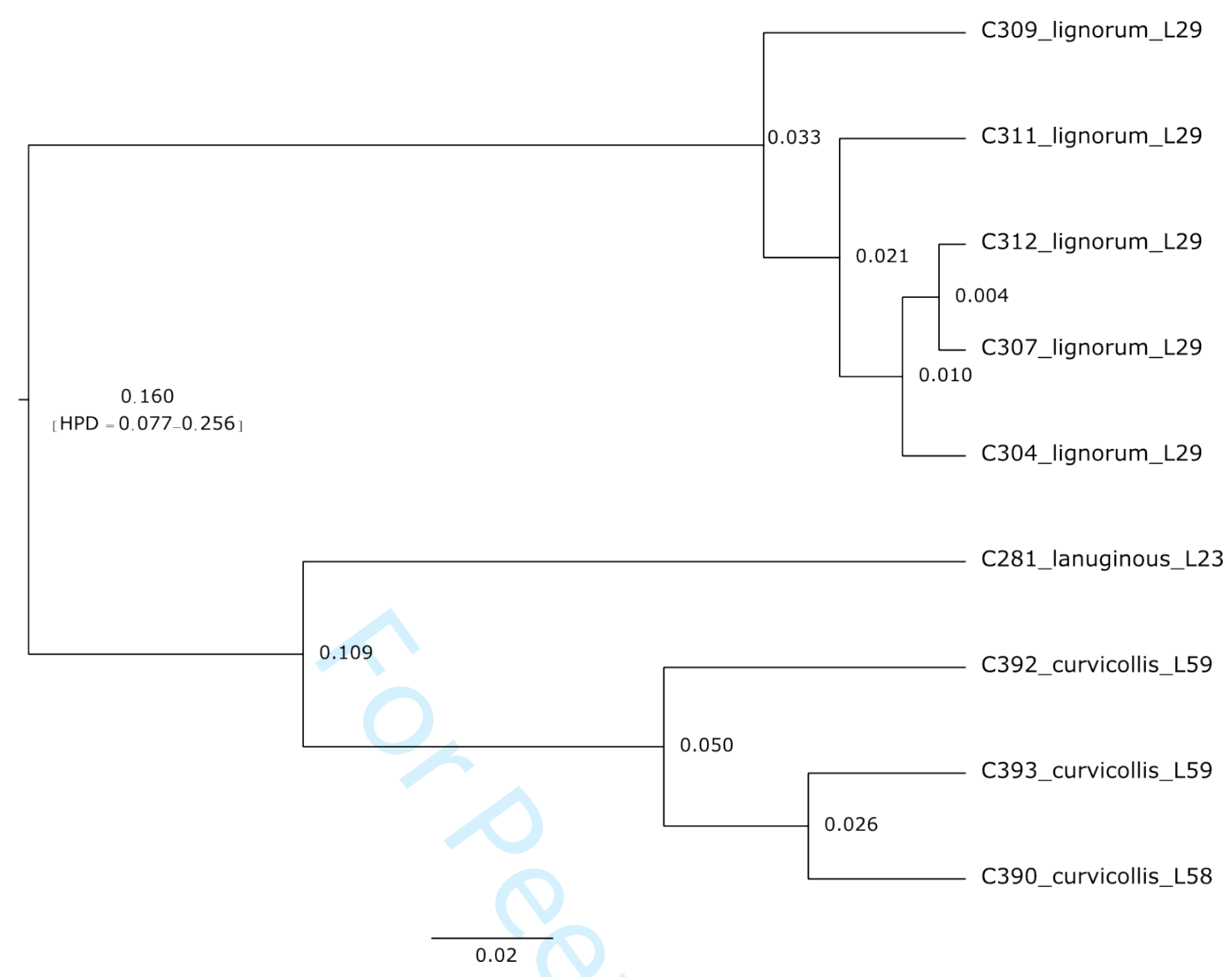

Figure S4.3. BEAST tree for Lepidocyrtus geographically localized monophyletic cluster OTU3. Numbers after the name indicate localities where sequences were sampled in Great Britain (Table S1). Node ages were estimated using a Collembola COI rate of 0.0421 substitutions/site/Ma (see text for details). 


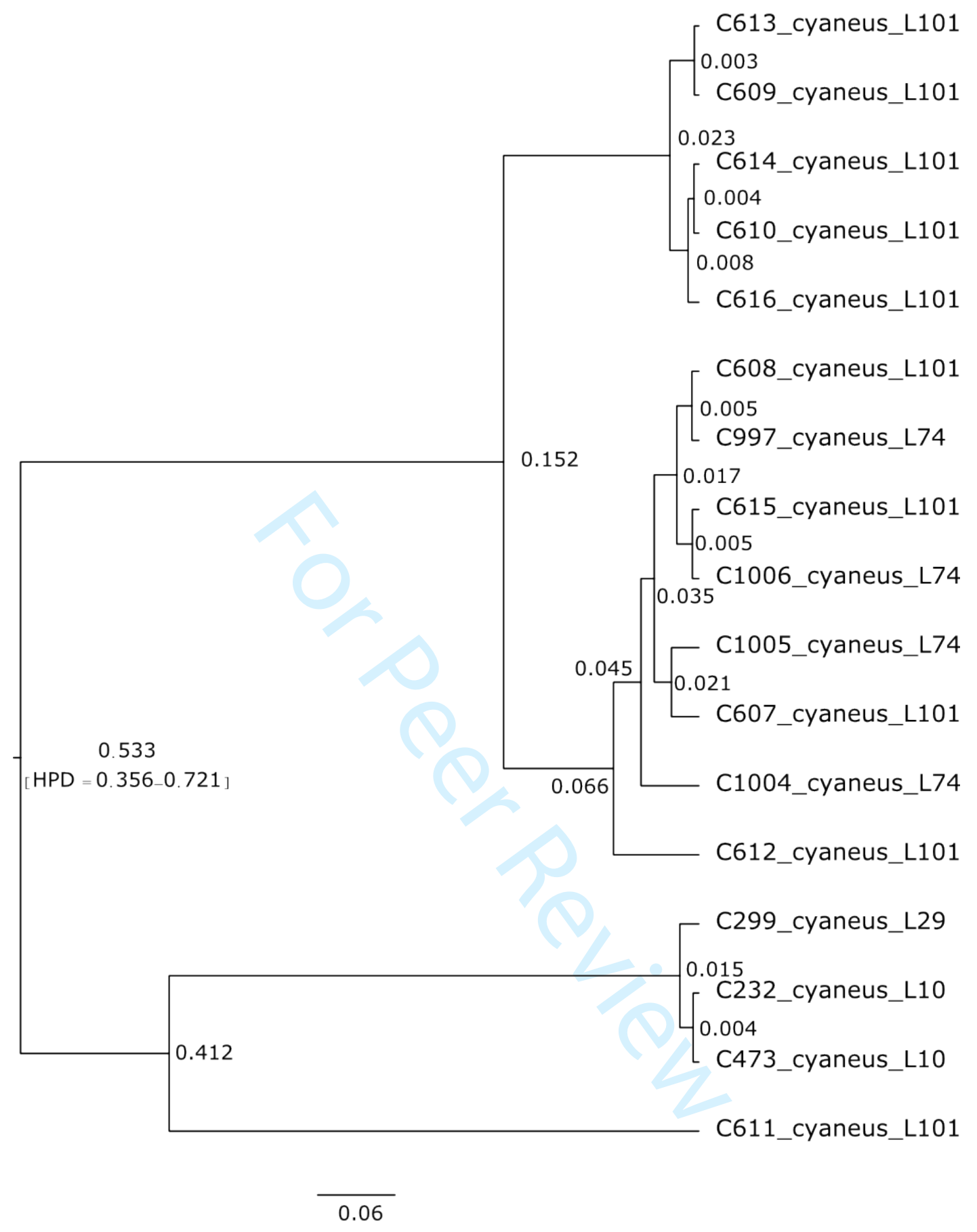

Figure S4.4. BEAST tree for Lepidocyrtus geographically localized monophyletic cluster OTU7. Numbers after the name indicate localities where sequences were sampled in Great Britain (Table S1). Node ages were estimated using a Collembola COI rate of 0.0421 substitutions/site/Ma (see text for details). 


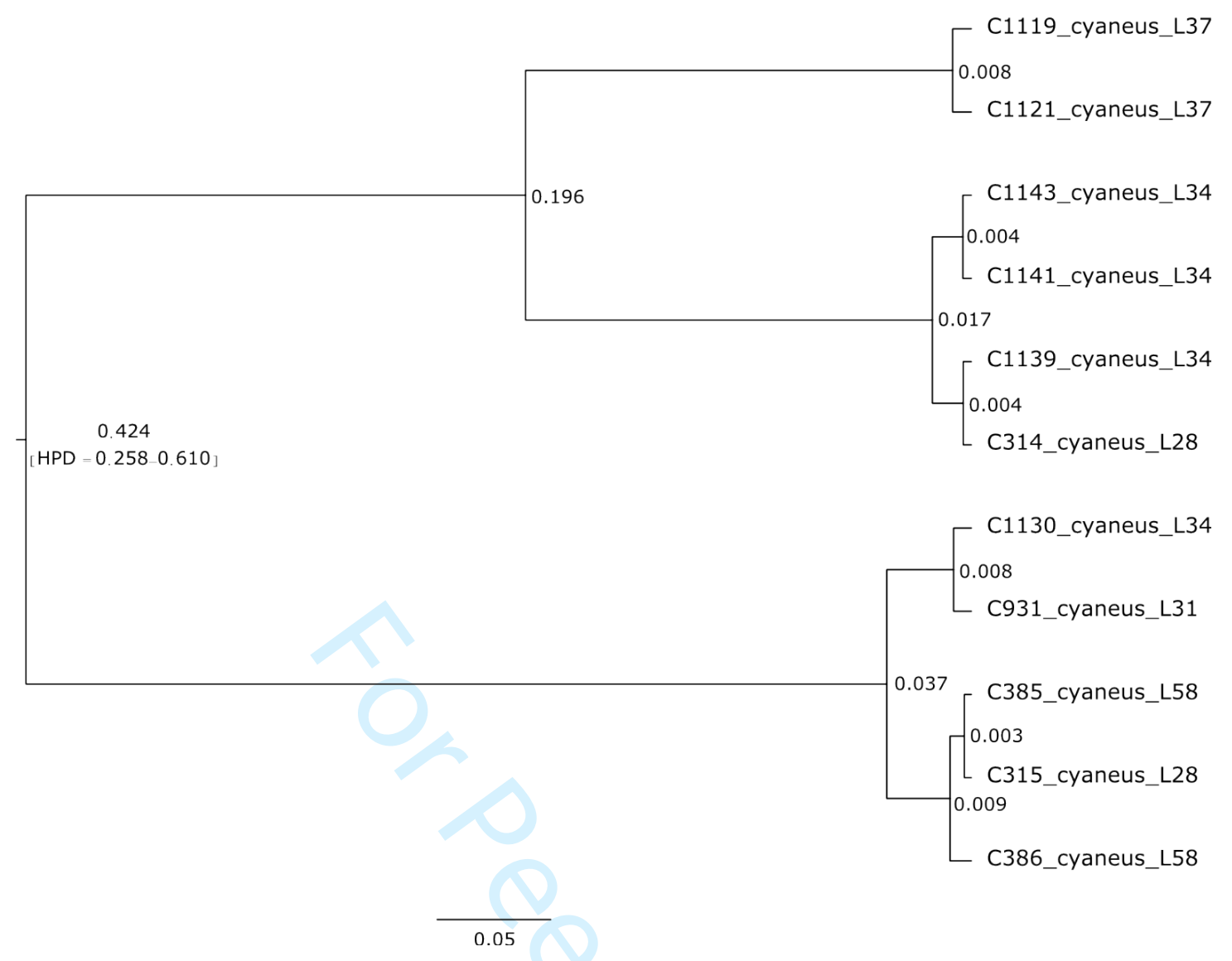

Figure S4.5. BEAST tree for Lepidocyrtus geographically localized monophyletic cluster OTU8. Numbers after the name indicate localities where sequences were sampled in Great Britain (Table S1). Node ages were estimated using a Collembola COI rate of 0.0421 substitutions/site/Ma (see text for details). 


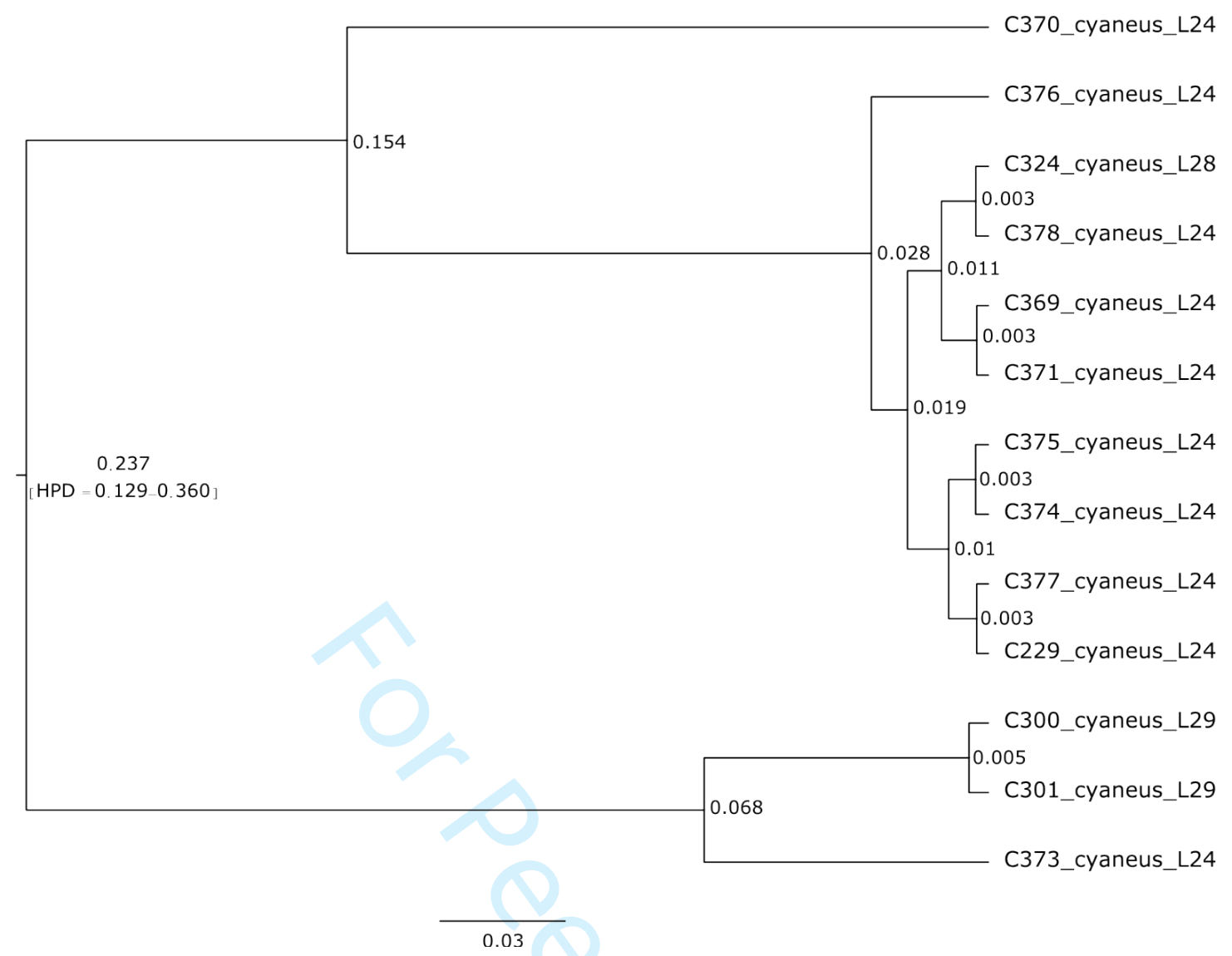

Figure S4.6. BEAST tree for Lepidocyrtus geographically localized monophyletic cluster OTU12. Numbers after the name indicate localities where sequences were sampled in Great Britain (Table S1). Node ages were estimated using a Collembola COI rate of 0.0421 substitutions/site/Ma (see text for details). 


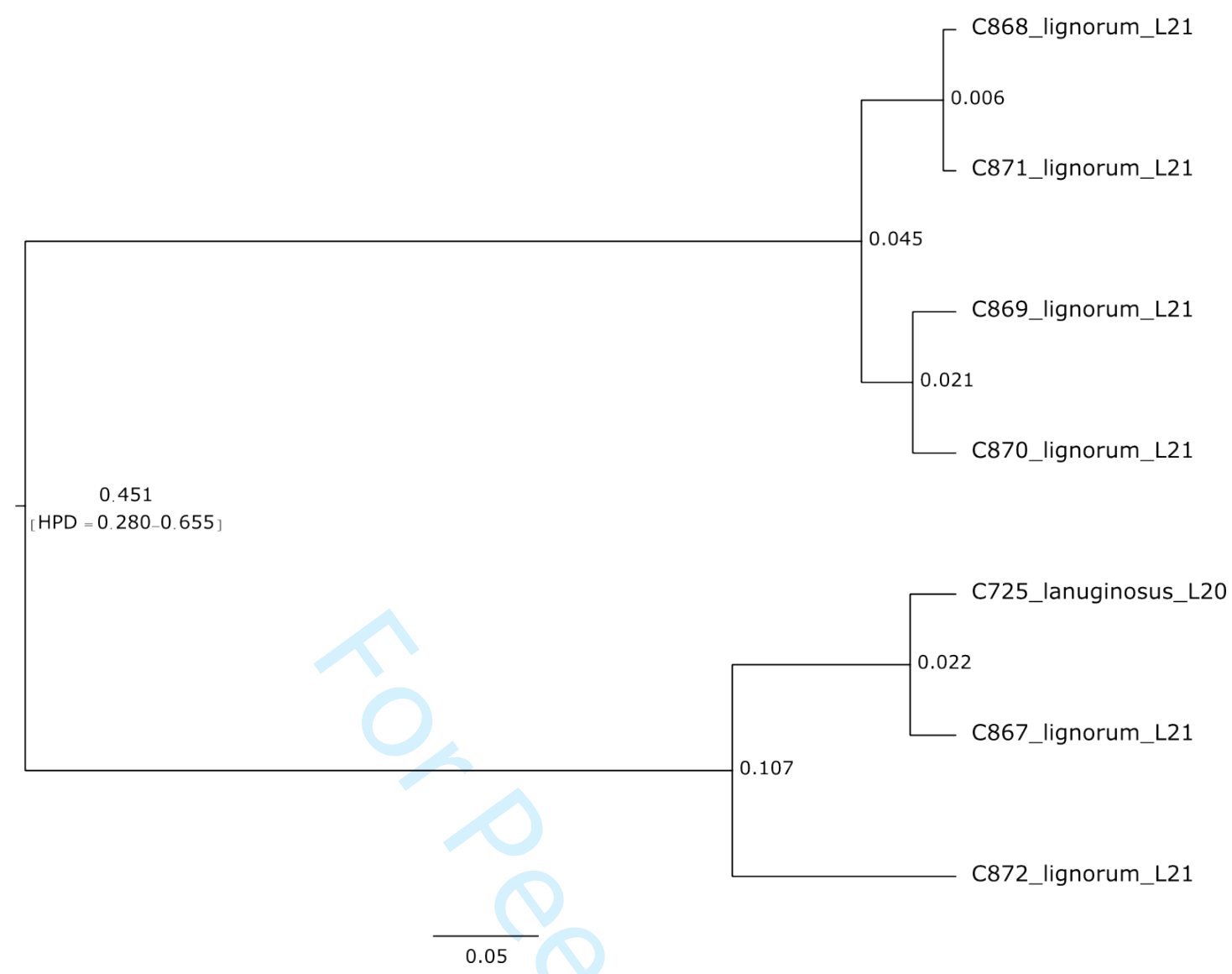

Figure S4.7. BEAST tree for Lepidocyrtus geographically localized monophyletic cluster OTU18. Numbers after the name indicate localities where sequences were sampled in Great Britain (Table S1). Node ages were estimated using a Collembola COI rate of 0.0421 substitutions/site/Ma (see text for details). 